\title{
INFLUENCE OF CROP LOAD ON FRUIT COMPOSITION USING PINOT NOIR GRAPES
}

\author{
A Thesis Presented to \\ The Faculty of California Polytechnic State University
}

San Luis Obispo, California

In Partial Fulfillment of the Requirements for

The Degree of Master of Science in Agriculture

By

Patrick Gregory Phelan

November 2009 
2009

Patrick Gregory Phelan ALL RIGHTS RESERVED 


\section{COMMITTEE MEMBERSHIP}

TITLE: Influence of Crop Load on Fruit Composition of Pinot Noir Grapes

AUTHOR: Patrick G. Phelan

DATE SUBMITTED: November 2009

COMMITTEE CHAIR: Dr. W. Keith Patterson, Professor Crop Science Department

COMMITTEE MEMBER: Dr. Steven Rein, Professor Statistics Department

COMMITTEE MEMBER: Dr. Brent Hallock, Professor Soil Science Department 


\begin{abstract}
Influence of Crop Load on Fruit Composition Using Pinot Noir Grapes

Patrick G. Phelan
\end{abstract}

November 2009

The two seasons for this trial were conducted in 2002 and 2003 at Bien Nacido Vineyard in Santa Maria, California. The trial consisted of pinot noir wine grapes clone 2a, grown on 10114 rootstock. There were eight treatments with a control consisting of 25 vines per treatment, and 50 vines for the control.

The primary objective of this research experiment was to conduct different vine balance procedures and compare them to the amount of wine phenolics. The vines were altered with two procedures. First was crop yield; fruit thinning altered the treatments by providing four different crop loads and a control. The treatment levels were signified as one ton, two ton, three ton, four ton, and control. The second procedure was timing of fruit thinning. Four different crop loads were split in half giving rise to eight treatments and a control. With four of the treatments the fruit was dropped post-bloom, and the other four treatments were dropped at seventy five percent veraison. Equal amounts of early and late thinning were combined and made into wine which gave four different crop load wines and a control wine. This process was done in 2002 and repeated in 2003. Additionally, other vine growth parameters were analyzed to determine the role that crop load and timing of thinning had in their development.

Seven variables were analyzed from prunings, clusters, juice, and wine samples. They consisted of berry size, cluster weight, titratable acidity, $\mathrm{pH}$, total soluble solids, phenolic profile 
(consisting of eleven components), and shoot weight. The results indicated that the following were statistically significant: (1) titratable acidity was significantly different between the 2002 and 2003 growing seasons, (2) total soluble solids showed a significant difference between the early fruit thinning versus the late thinning, (3) berry size in 2002 showed a statistically significant effect of drop date on the mean caliper size, (4) in 2002 the berry size also showed a statistically significant interaction between drop level and drop date, (5) berry size in 2003 showed a statistically significant effect of drop level on the mean caliper size, (6) in 2003 there is also a statistically significant effect of drop date on the mean caliper size, (7) again in 2003 there is a statistically significant interaction between drop level and drop date, (8) cluster weights in 2002 showed a statistically significant relationship between drop level and average cluster weight, (9) in 2002 cluster weights also showed an effect of the time of fruit drop on the average cluster weight, (10) cluster weights in 2003 showed a statistically significant relationship between drop level and the average cluster weight, (11) pruning weights in 2002 showed a statistically significant relationship between drop level and average shoot weight, (12) pruning weights in 2003 showed a statistically significant relationship between drop level and average shoot weight and finally, (13) of the eleven phenolic components measured, five differed significantly by year but not across the five treatments.

Information derived from this experiment suggests that the site is a high vigour location according to Robinson and Smart's yield to pruning weight ratio. With this in mind we find that TA and pH were not a significant factor, but soluble solids (brix) ascertained a higher degree of brix with the late drop treatment. We conclude this to be an effect of both the high vigour site and more uniform fruit drop. In 2002 and 2003, we find a significant interaction between drop date and berry size with a late drop producing the smallest berries on average. Cluster weights 
varied between 2002 and 2003 and the results were inconclusive. Shoot weights along with harvest totals concluded a high vigour site. The one ton level in 2002 had a significantly higher average shoot weight than the three ton level $(\mathrm{p}<.001)$, four ton level $(\mathrm{p}=.006)$, and no fruit drop control $(\mathrm{p}<.001)$. There is also a significant relationship between drop time and average shoot weight in $2002(\mathrm{p}<.001)$, and $2003(\mathrm{p}=.049)$ with the late fruit drop being followed by a lower shoot weight. This significant relationship expressed that an early drop increased the shoot weight which contributed only more vigour to an already high vigour site. The ratio of yield to pruning weight for 2002 gave us a range of .39 to 1.78 , and in 2003 the range was .32 to 1.36 concluding a high vigour site. The phenolic profile expressed a statistical difference by year, but was most likely caused by a varying fermentation temperature or different climatic conditions in 2002 and 2003. 


\section{ACKNOWLEDGMENTS}

I wish to thank the many people who saw me through this long journey. First and foremost I would like to thank my wife Colby Phelan, and my children, Neely and Jeffrey Phelan, for the time lost with them in order to complete this thesis. I would also like to give thanks to my advisor Keith Patterson for accepting the many phone calls and delays to this project. A special thank you goes to the Miller family for allowing me to perform the trial on the Bien Nacido Vineyard property. Thanks to Gordon Burns of ETS laboratories for the student pricing he gave me on two phenolic profiles. Thanks to the vineyard staff at Bien Nacido, especially Chris Hammell for the time and effort put forth in helping me collect all the data needed for the project. 


\section{TABLE OF CONTENTS}

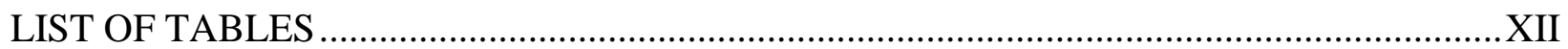

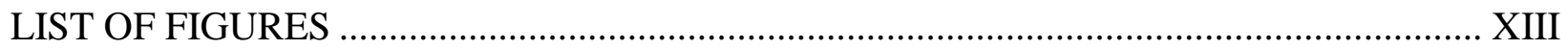

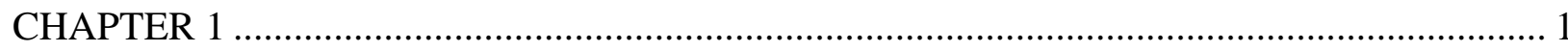

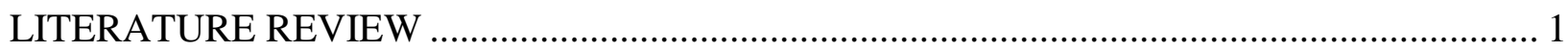

Pinot Noir Clone 2A.

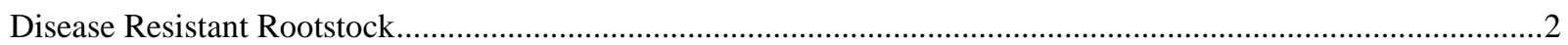

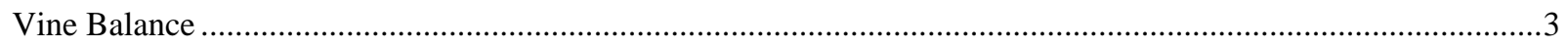

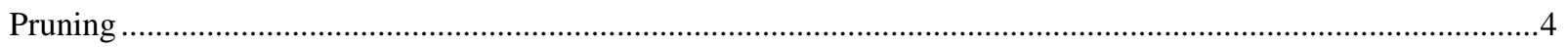

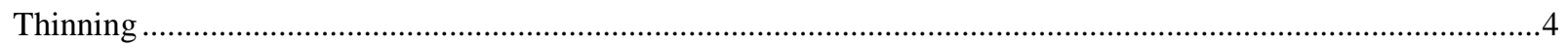

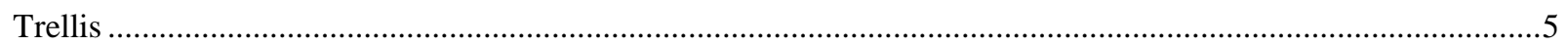

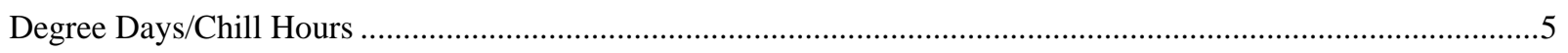

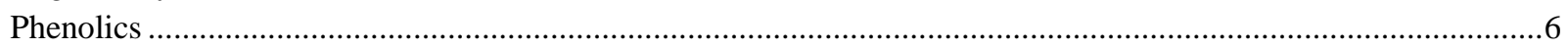

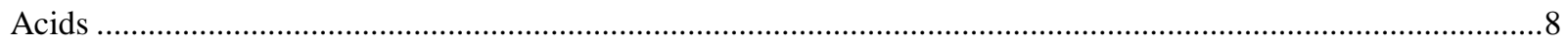

$\mathrm{pH}$

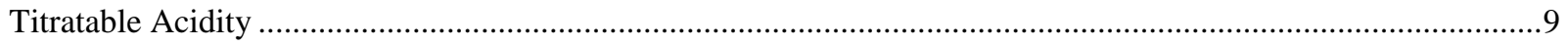

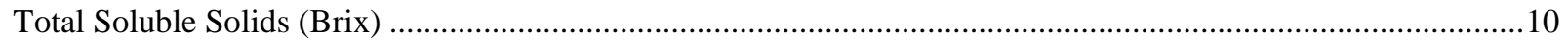

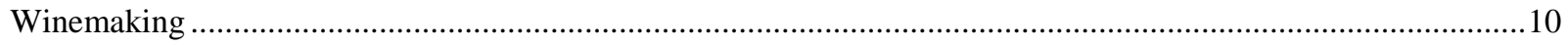

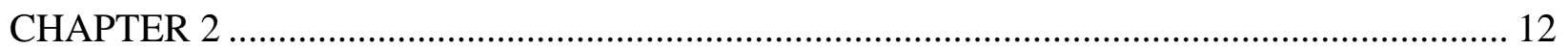

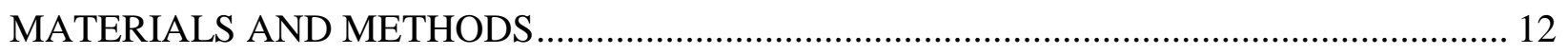

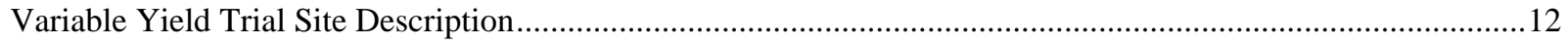

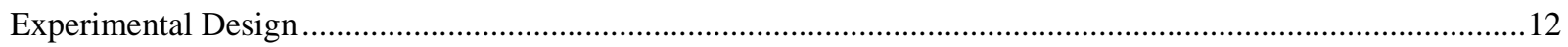

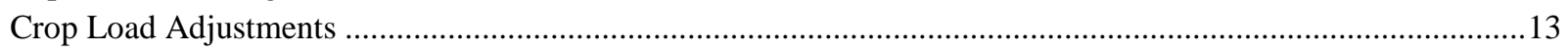

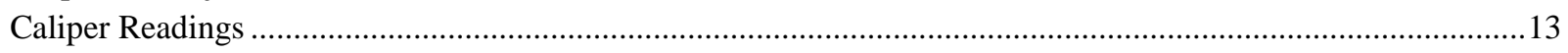

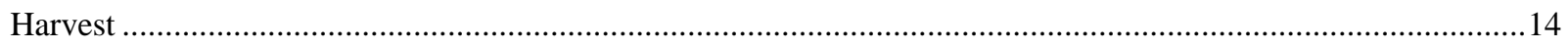

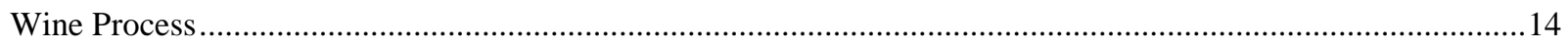

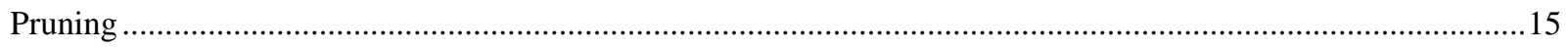

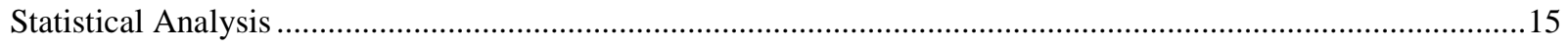

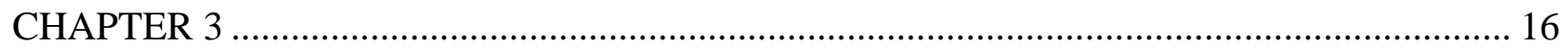

RESULTS AND DISCUSSION ................................................................................ 16

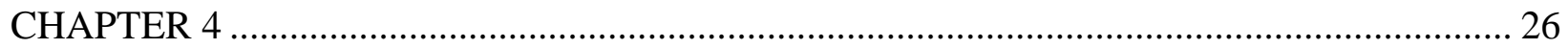

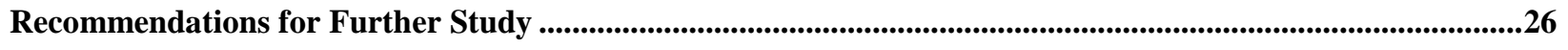




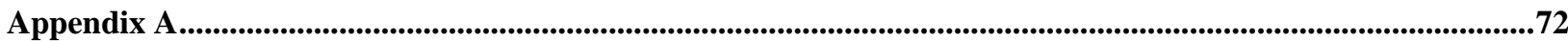

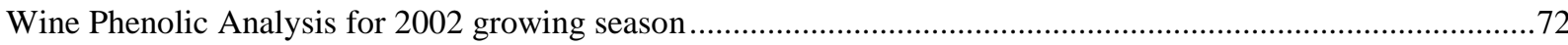

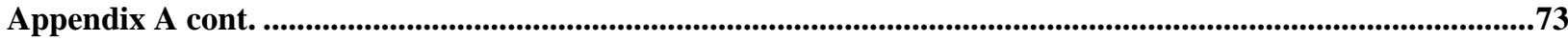

Wine Phenolic Analysis for 2002 growing season ...........................................................................................73

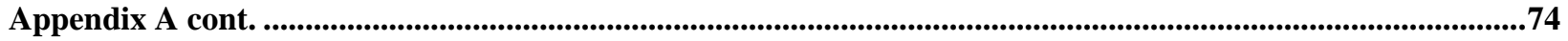

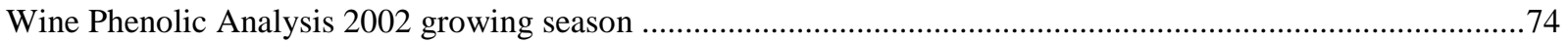

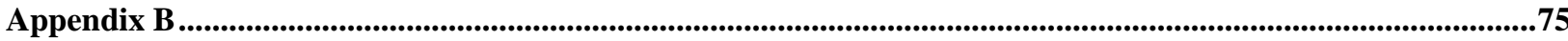

Wine Analysis 2002 growing season 1 ton treatment compared to 5 ton control ................................................75

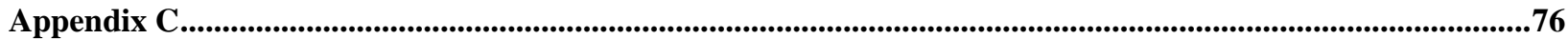

Wine Analysis 2002 growing season 2 ton treatment compared to 5 ton control ..............................................76

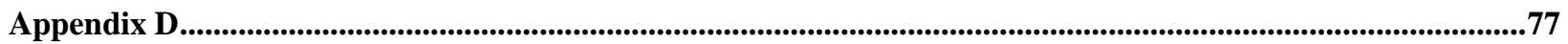

Wine Analysis 2002 growing season 3 ton treatment compared to 5 ton control ...............................................77

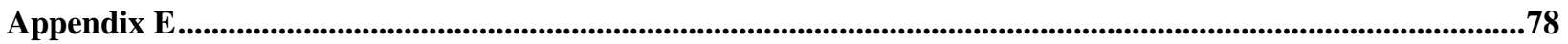

Wine Analysis 2002 growing season 4 ton treatment compared to 5 ton control ..............................................78

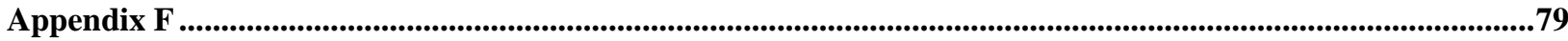

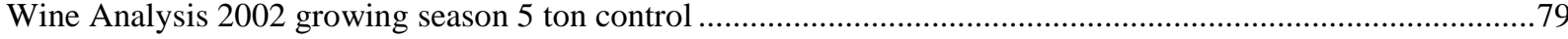

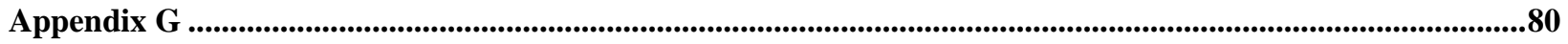

Wine Phenolic Analysis 2003 growing season .................................................................................................. 80

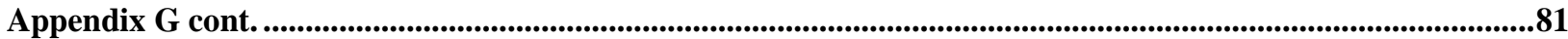

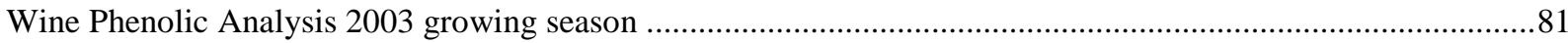

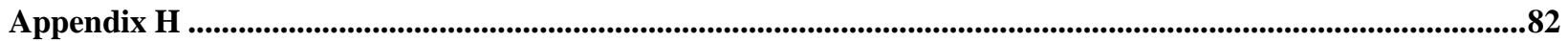

Wine Analysis 2003 growing season 1 ton treatment compared to 5 ton control .............................................82

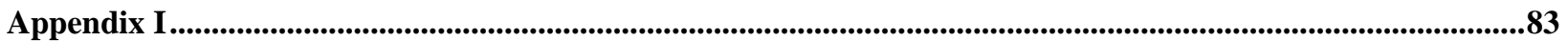

Wine Analysis 2003 growing season 2 ton treatment compared to 5 ton control ...............................................83

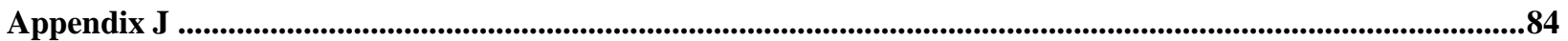

Wine Analysis 2003 growing season 3 ton treatment compared to 5 ton control ............................................84

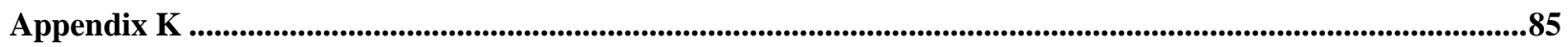

Wine Analysis 2003 growing season 4 ton treatment compared to 5 ton control ...............................................85

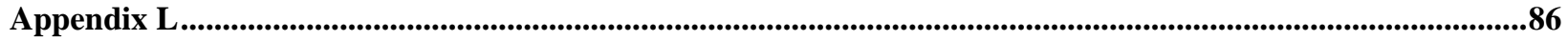

Wine Analysis 2003 growing season 5 ton control ……...............................................................................8 
Appendix M.

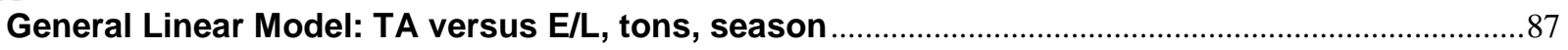

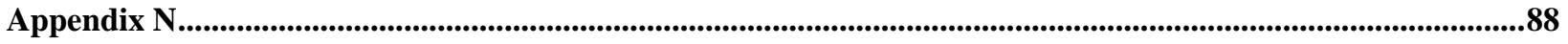

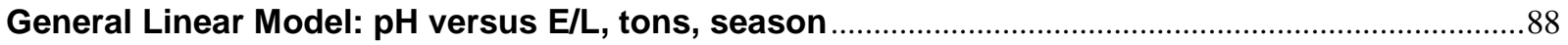

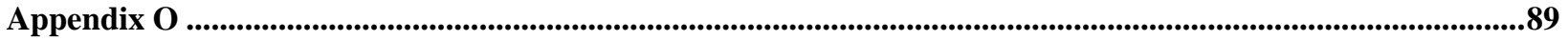

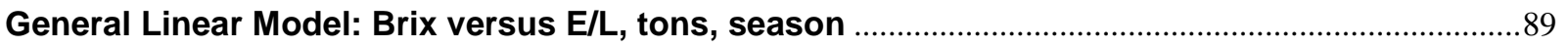

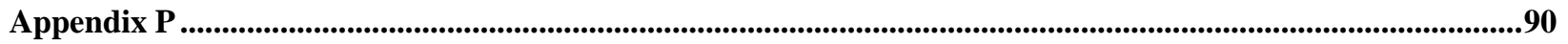

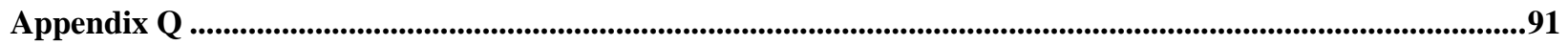

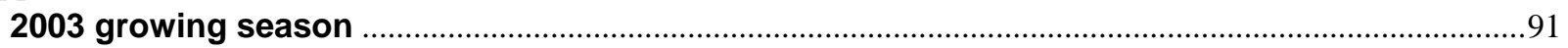

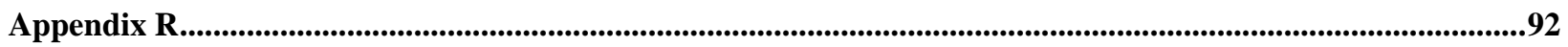

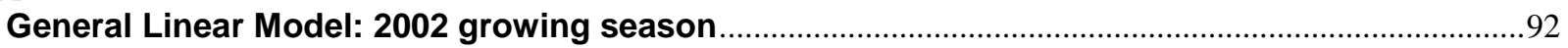

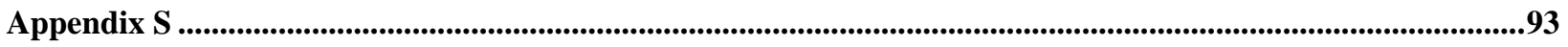

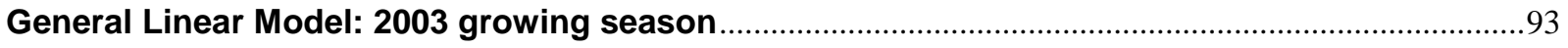

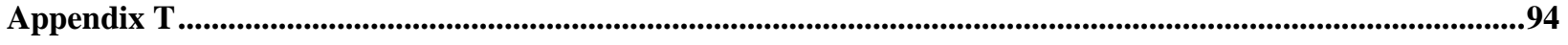

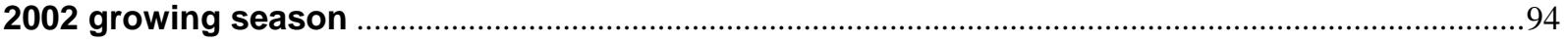

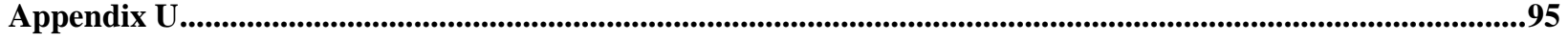

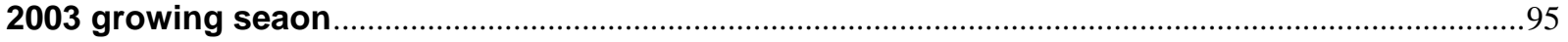

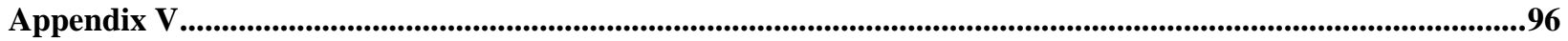

General Linear Model: gallic acid versus Treatment, Year.............................................................96

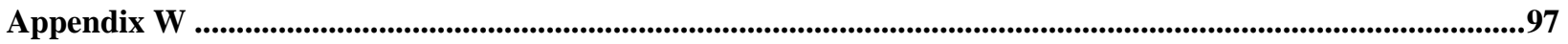

General Linear Model: catechin versus Treatment, Year ..............................................................97

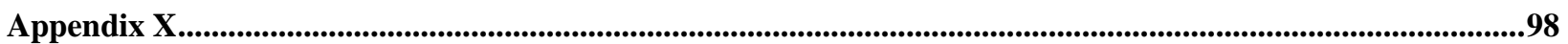

General Linear Model: epicatechin versus Treatment, Year ........................................................98

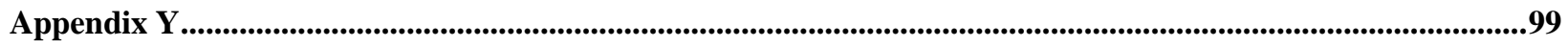

General Linear Model: polymeric phenols versus Treatment, Year ...............................................99

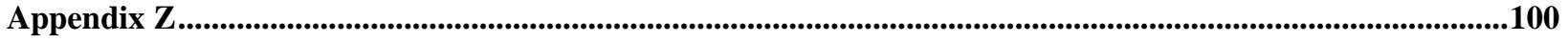

General Linear Model: caftaric acid versus Treatment, Year.............................................................100

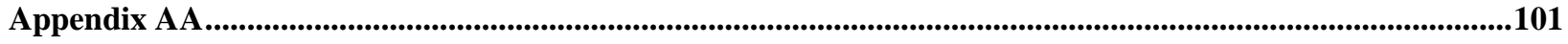

General Linear Model: caffeic acid versus Treatment, Year ......................................................... 101

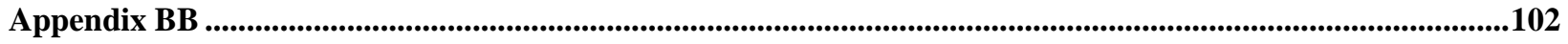

General Linear Model: quercetin glycosides versus Treatment, Year .........................................102

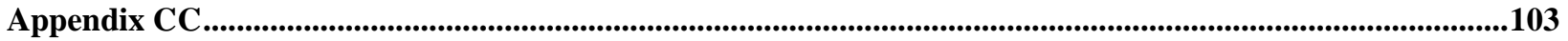

General Linear Model: quercetin aglycone versus Treatment, Year............................................103 


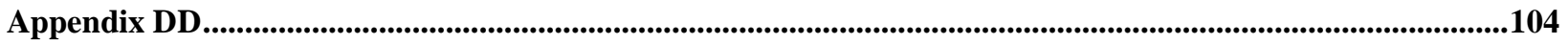

General Linear Model: malvidin glucoside versus Treatment, Year.....

Appendix EE ..........................................................................................................................................................105

General Linear Model: polymeric anthocyanins versus Treatment, Year ......................................105

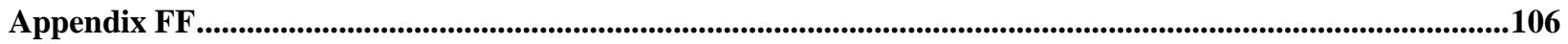

General Linear Model: total anthocyanins versus Treatment, Year ..................................................106

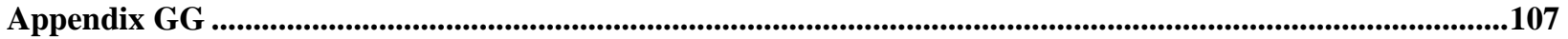

General Linear Model: monomeric anthocyanins versus Treatment, Year.....................................107 


\section{LIST OF TABLES}

Table

Page

1. Harvest results from 2002 and 2003 growing seasons 64

2. Pruning results from 2002 and 2003 growing seasons 65

3. Pruning : Fruit Ratios for 2002 growing season 66

4. Pruning : Fruit Ratios for 2003 growing season 67 


\section{LIST OF FIGURES}

Figure $\quad$ Page

1. Degree hours accumulated from Adcon weather station in 2002 and $2003 \_28$

2. Chill hours accumulated from Adcon weather station in 2002 and $2003 \_29$

3. Weather data from Adcon weather station in 2002 and 2003

4. Photographs of trial site and two ton treatment___ 31

5. Photographs of one ton treatment and five ton control treatment 32

6. Photographs of pruned three ton treatment and entire pruned trial site ___ 33

7. Map layout of trial site___ 34

8. 2002 growing season map containing number of canes pruned/vine __ 35

9. 2002 growing season map containing ounces of prunings/vine___ 36

10. 2002 growing season map containing number of clusters harvested/vine ___ 37

11. 2002 growing season map containing ounces of harvested fruit/vine___ 38

12. 2003 growing season map containing number of canes pruned/vine __ 39

13. 2003 growing season map containing ounces of prunings/vine___ 40

14. 2003 growing season map containing number of clusters harvested/vine __ 41

15. 2003 growing season map containing ounces of fruit harvested/vine___ 42

16. Interaction plot for TA from 2002 and 2003 growing seasons___ 43

17. Interaction plot for $\mathrm{pH}$ from 2002 and 2003 growing seasons 44

18. Interaction plot for Brix from 2002 and 2003 growing seasons 45

19. Interaction plot for berry size from 2002 growing season___

20. Interaction plot for berry size from 2003 growing season___ 47

21. Interaction plot for average ounces/cluster/vine for 2002 growing season___ 48

22. Interaction plot for average ounces/cluster/vine for 2003 growing season___ 49

23. Interaction plot for average ounces/shoot/vine for 2002 growing season__ 50

24. Interaction plot for average ounces/shoot/vine for 2003 growing season___ 51

25 . Interaction plot of gallic acid versus ttt__ 52

26. Interaction plot of catechin versus ttt__ 53

27. Interaction plot of epicatechin versus $\mathrm{ttt}$

28. Interaction plot of polymeric phenols versus ttt__ 55

29. Interaction plot of caftaric acid versus ttt__ 56

30. Interaction plot of caffeic acid versus ttt_ 557

31. Interaction plot of queretin glycosides versus ttt__ 58

32. Interaction plot of quercetin aglycone versus ttt

33. Interaction plot of mavidin glucoside versus ttt__ 60

34. Interaction plot of polymeric anthocyanins versus $\mathrm{ttt} \_61$

35 . Interaction plot of total anthocyanins versus ttt___ 62

36. Interaction plot of monomeric anthocyanins versus $\mathrm{ttt} \_63$ 


\section{Chapter 1}

\section{Literature Review}

Pinot noir is an old red grape variety dating back to the first century AD [3]. Pinot noir belongs to the vitis vinifera species which accounts for ninety percent of the world's grapes [30]. Among the many different clones there are many differences in growth habit, berry size, bunch compactness and leaf shape [10]. Bunch compactness, found more prevalent in certain clones of pinot noir, gives rise to bunch rot. Bunch rot, Botrytis cinerea, is a fungal disease which attacks the succulent tissue, particularly the floral parts or ripening fruit [10]. Budburst in pinot noir is earlier than other varieties, which makes it susceptible to spring frost damage. Pinot noir is most prevalent in Burgundy France, which is known for fine dry wine, and in Champagne France it is known for its rose' champagnes [3]. No information has stated an exact number of pinot noir clones but some authors have claimed the existence of more than a thousand different clones [26].

\section{$\underline{\text { Pinot Noir Clone 2A }}$}

Within a single grape variety, there is a rich variation of subtle genetic differences manifested by their growth habit and grape berry character [9]. Concerns about grapevine health, in addition to fruit quality, have led to a process called clonal selection. In viticulture, a clone is a population of vines derived by vegetative propagation from a single vine, called a mother vine. All vines grown from cuttings or buds of this vine are genetically identical. Future generations will remain identical unless a spontaneous mutation occurs, creating a bud with an altered genetic makeup [9]. 
In the 1940's, Professor Olmo made several trips to Europe where he collected Pinot noir cuttings from many different vineyards [9]. These vineyards were located in Germany (Geisenheim selection), France (Pommard selections), and Switzerland (Wadsenwil selection). The Pinot noir clone 2A was sourced from the mother vine in Wadsenwil, Switzerland [9]. A clonal trial was conducted in Oregon at two different vineyards located in the Willamette Valley. The trial took place in 1985 and 1986 by the viticulturists Steve Price and Barney Watson, which involved the Pinot noir clone $2 \mathrm{~A}$ besides many others. The findings from the clone $2 \mathrm{~A}$ demonstrated a correlation between low yield and high varietal intensity [9].

\section{Disease Resistant Rootstock}

Viticulture in the United States, as well as most wine grape growing regions, is based on varieties of the European vine, Vitis vinifera. This species is particularly prone to attack by two soil-borne pests, the grape phylloxera and plant parasitic nematodes [30]. It has been found that the European vine can be protected from these pests by grafting to rootstock varieties derived from other vine species and hybrids that are resistant. Many of the rootstocks used for this purpose are well adapted to particular soil types and some may also be used to overcome vineyard problems such as drought and salinity [30].

The phylloxera is native to the Mississippi Valley where resistant wild varieties were found. These American varieties were Vitis riparia, Vitis rupestris, Vitis berlandieri, and many more [30]. A hybrid of riparia and rupestris was obtained by Millardet in 1882 [22]. The hybrid of this is known today as 101-14 Millardet et de Grasset and is widely used throughout the industry. The 101-14 rootstock is a low to moderate vigor rootstock with much of its use in poorly drained shallow soils [22]. Its low to moderate vigor is much like 3309C which is a hybrid of riparia and rupestris as well [22]. The 101-14 rootstock does not do well in dry grape- 
growing areas and has moderate resistance to nematodes. The 101-14 rootstock can only tolerate up to nine percent active lime in the soil [22].

\section{$\underline{\text { Vine Balance }}$}

There are three major categories, developmental, cultural, and environmental, which have a direct effect on vine balance. Vine balance is the ratio between fruitfulness and vegetative growth. The amount of crop that a vineyard can produce is essentially dependent on its exposed canopy surface area. The ideal ratio of yield to canopy surface area is $0.8 \mathrm{~kg} / \mathrm{m} 2$ in cool climates for medium ripening varieties. Such measurements are considered essential to ensure vine balance. This is in a quantitative form, so that vineyard management practices can be monitored in relation to vine balance and ultimately wine quality [18].

The discussion of vine balance has raged for many years, and is not a new idea. Ravaz is the earliest source of relevant information, and the Ravaz Index suggests that the ratio of fruit to wood is the key to achieving both fruit quality and consistent production. He also showed a general relationship between leaf production and fruit production. As noted by Ravaz, Partridge, Shaulis, and subsequent re-searchers, balance may also be considered as the amount of leaf area required to ripen a unit of crop weight [14].

Yield at harvest, and cane weights at pruning are the most informative of all the measurements which can be made to indicate vine balance [27]. Pruning weight is proportional to leaf area carried on the shoots the previous growing season. Calculation of mean cane weight gives a useful indication of shoot vigor. Measurements of yield and cluster number allow calculation of mean cluster weight. The ratio of yield to pruning weight gives a good indication 
of balance between fruit and vegetative growth. An optimal level for moderate vigor vines is a yield/pruning weight ratio of 5-10g [27].

\section{Pruning}

The cultural practice of pruning in the winter months is extremely important when trying to balance fruitfulness to vigor. Head, cane, and spur pruning styles exist and are the foundation to vine balance [27]. The more nodes/buds left behind at pruning is a direct correlation to possible fruitfulness which can be used to devigor a vigorous vine by leaving behind many buds and vice versa for a weak vine [10].

\section{Thinning}

Thinning vines increases the leaf area to fruit ratio and provides for an earlier harvest date. Thinning also appears to have a strengthening effect on the vines that is measured as greater ripe nodes of periderm and earlier leaf area development than standard vines [2].

The canopy's ability to ripen fruit, as noted by Ravaz, Partridge, Shaulis and subsequent researchers, show that balance may also be considered as the amount of leaf area required to ripen a unit of crop weight. This is commonly expressed as $\mathrm{cm} 2$ leaf area/gm fresh weight of fruit. The literature reports a range of 7 to $14 \mathrm{~cm} 2 / \mathrm{gm}$ to achieve ripening [14].

The amount of crop that a vineyard can produce is essentially dependent on its exposed canopy surface area. The ideal ratio of yield to canopy surface area is $0.8 \mathrm{~kg} / \mathrm{m} 2$ in cool climates for medium ripening varieties [18]. The vine's response to thinning is a physiological one, in which we are changing the ratio of exposed leaf area to mass of fruit. The target is to achieve fifteen $\mathrm{cm} 2 / \mathrm{g}$ of fruit according to Melsom [18]. Other factors that can impact vine physiology 
and have an impact on the response to thinning are drought stress, physical injury, damage from pests and disease [29].

\section{$\underline{\text { Trellis }}$}

A major developmental factor is choosing the right trellis system for the variety of grape to be planted. Pinot noir has had tremendous results and is widely planted on the vertical shoot positioning trellis (V.S.P.) [27]. This trellis can be a spur or cane pruned depending on the yield desired. The vertical shoot positioning trellis is an excellent trellis for light exposure and is intended for vines with moderate growth characteristics. The V.S.P. trellis has also been adopted in places where there is a high fungal disease pressure since good air circulation exists within the canopy of V.S.P. [27].

\section{$\underline{\text { Degree Days/Chill Hours }}$}

Degree days are the positive remainders when a plant-specific base temperature (commonly $10^{\prime} \mathrm{C}$ or 50'F) is subtracted from daily average temperatures. Accumulations of degree days are thought to provide a general indication of thermal conditions in a particular viticulture region [17]. The accumulation approximates the same total at specified stages of vine development. This can be a useful indicator of season length [17].

Pinot noir is a cool climate variety which grows best in region one or region two climate. Region 1 climate is 2500-degree days or less and region 2 climate is 2501-3000 degree days [30].

As grape canes mature, their buds enter a type of dormancy in which their growth is suppressed. Repeated exposure to cold temperatures sustains this form of dormancy. Once the chilling hour requirement is satisfied, it is only low temperatures which prevent bud burst. The 
number of chilling hours required by grapes varies among the different varieties, but most grapes only need about 150 chilling hours $[28,1]$.

In California, we typically calculate chilling hours from November 1, through the end of February. Cumulative chilling hours are calculated by either summing the hours below 45 degrees or those between 32 degrees and 45 degrees. The hours can be recorded using a purchased weather station, such as an Adcon station, or by using one of several Web sites which calculate hours from weather stations nearest your vineyard [28]. A common chill accumulation model is the $450 \mathrm{~F}$ and Under Model, which uses " 1 hour $<=450 \mathrm{~F}=1.0$ chill unit" [1].

\section{Phenolics}

Grapes and wines form a diverse class of phenolic compounds derived from a hydroxybenzene ring, a basic phenol structure [31]. Nonflavonoid phenols, flavonoid phenols, and complex phenols can summarize the phenol substances of wine. These phenolic substances are the essential components responsible for wine color (red pigments or brown-forming substrates), and mouth feel, which can be further broken down to astringency and bitterness. When these phenolics are oxidized, they can later participate in further reactions in the wine, such as the precursor to 4-ethyl-phenol, an undesirable sensory component attributed to spoilage yeast brettanomyces [31].

Nonflavonoid phenols, or phenolic acids, can be further subdivided into two groups, hydroxycinnamic and hydroxybenzoic acids. Hydroxycinnamates are derivatives of cafeic, pcoumaric and ferulic acids, which in grape juice are esters bonded with tartaric acid known as caftaric, coutaric, and fertaric acids [7]. The hydrolysis of non-flavonoid esters, in the presence of an esterase, occurs during alcoholic fermentation and results in ethyl phenols most importantly 
4-ethyl phenol [31]. Gallic acid, being the most important of hydroxybenzoic acids, increases with pomace contact in red wines. Gallic acid has been given great attention due to its chemical structure's ability to accept, stabilize, and release the oxidation state forms of NO (nitric oxide), a molecule suspected of allowing blood vessels to relax and permit better blood flow [32]. Nonflavonoids are important in forming golden colors for white wine [6].

Flavonoid phenols that are found in skins, seeds, and pulp of the fruit contribute to the structure and color of wine. The major groups of flavonoids are flavan-3-ols, flavan-3, 4-diols, flavonols, and anthocyanins. Flavan-3-ols are primarily (+)-catechin and (-)-epicatechin . Catechins are precursors to browning in white wines and bitterness in red wines [32]. The polymeric forms of flavan-3-ols can be referred to as condensed tnnins. Flavan-3, 4-diols is a group important to winemakers due to the influence it has on pinking often seen in white wines. Flavonols occur in glycosidic forms in grape skins, which can attribute to a red wine's bitterness [32]. Plant pigments posses a large group known as anthocyanins, which give red grapes its color.

Complex tannins, or complex phenols, are polymers of both flavonoid and non-flavonoid phenols. These polymers have been given the generic term, "tannins". Hydrolyzed flavonoid phenols exist as esters and can be classified as hydrolysable tannins. Condensed tannins cannot be easily decomposed by hydrolysis. The astringent tannins are the result of the formation of blue-color complexes reacting with Fe3+, which reacts with protein [32]. The phenolic compounds encouraging these reactions typically have a molecular weight from 500 to 5,000, however, as the wine ages and further polymerization occurs molecular weights range from 2,000 to $5,000[32]$. 


\section{$\underline{\text { Acids }}$}

Acidity plays a vital part in determining wine quality. Lack of acid will produce poor fermentation, medicinal taste and a flabby, not vibrant feeling on the palate. In any wine it is essential that acidity, tannin content and sweetness be in balance with the type of wine you are making [21]. Ninety percent of acids in wine are malic and tartaric acids $[15,20,24]$. The remaining ten percent are found in small quantities. They are: galacturonic, glucuronic, gluconic, oxalic, mucic, citramilic, dimethyglyceric, pyruvic, ketoglutaric succinic, lactic, acetic, and citric, which is the primary acid in most fruits other than grapes [21]. The favorable, soft tasting, tartaric acids are rarely found in any other fruit except grapes and pineapples. It is considered the strongest acid and the amount present in the grape has the greatest effect on $\mathrm{pH}$ [21]. Tartaric acid respires slowly, if at all, and concentrations are reduced during ripening by dilution with water due to berry growth $[10,20]$. The other dominate acid found in wine, malic acid, is fragile and is easily metabolized. Decreased total acidity in the grape is attributed to decreased malic acid content. Malic acid levels also metabolize faster in warmer climaties compard to cooler climates because of increased respiration [10,20,21,24]. Malic acid can be reduced or eliminated by inoculating into the wine selected lactic acid bacteria strains such as Pediococcus, Lactobacillus, and Leuconostoc $[13,16,24]$. This process is known as malo-lactic fermentation, and is a bioconversion of malic acid to lactic acid and carbon dioxide. Not only will malo-lactic fermentation de-acidify the wine, but has also proven to improve the biological stability of wine [4]. Malo-lactic fermentation can also occur spontaneously after alcoholic (yeast) fermentation due to the existence of micro-organisms found in the cellar of fermentation vats $[13,24]$. 
The $\mathrm{pH}$ (true acidity) of wine is an important chemical parameter in premium wine production. High $\mathrm{pH}$ can have a negative effect on: microbiological stability, potassium bitartrate stability (cold stability), calcium stability, protein stability, color, oxidation rate, and tannin solubility $[25,31]$. Similar to titratable acidity, $\mathrm{pH}$ also measures free hydrogen ions, but does not include un-dissociated forms of organic acids. This is why $\mathrm{pH}$ only measures values that can be tasted $[19,24]$. The $\mathrm{pH}$ measurement is based on a logarithmic scale. An example, $\mathrm{pH}$ of 2.0 is ten times more acidic than a $\mathrm{pH}$ of 3.0 [31]. Therefore, small numerical differences in $\mathrm{pH}$ correlate with large reactivity and flavor differences [6]. Mathematically, $\mathrm{pH}$ is defined as subscript ten of the concentration of hydroxonium ions in an electrically conductive solution: $\mathrm{pH}$ $=-\log 10[\mathrm{H} 3 \mathrm{O}+][23]$. The $\mathrm{pH}$ is measured with a $\mathrm{pH}$ meter that has two glass electrodes, generally calibrated at $\mathrm{pH} 4$ and $\mathrm{pH} 7$ known as a two-point calibration [19, 24, 25]. At pH 7.0 the juice and wine solution is neutral, below it is increasingly acidic and tart to a minimum of zero, and above increasingly alkaline to a maximum of fourteen [6].

\section{Titratable Acidity}

The titratable acidity (TA), also known as total acidity, present in wine and juices is a measure of the concentration of free hydrogen ions plus any un-dissociated forms of acids, which include volatile acids, inorganic acids such as phosphoric acid, organic acids, and amino acids. It is difficult to predict TA of a wine based on the acidity of the juice because yeasts and bacteria consume acids. However, yeast and bacteria also produce acids, for instance, succinic and lactic acids [25]. Titration analysis uses standardized sodium hydroxide to the pink endpoint of $\mathrm{pH}$ 8.2. Furthermore, TA is expressed on the basis of grams tartaric acid per liter $[6,10,19,24,25$, $31]$. 


\section{$\underline{\text { Total Soluble Solids (Brix) }}$}

Total soluble solids (sugar) are often measured, among other methods, with a refractometer or a hydrometer. The refractometer is an instrument that measures juice weight by utilizing a calibrated prism. When held against the light, a drop of juice on the prism causes light to be refracted in proportion to the concentration of sugar in the juice. Similarly, the hydrometer also measures juice weight, but the juice is put into a graduated cylinder and the lines on the floating hydrometer correspond to the density of the juice. Both methods measure Brix or Balling, based on the fact that grape juice is heavier than water. One milliliter of water weighs one gram, thus the excess weight of one milliliter of juice is the weight of the substances dissolved in it. Fortunately, ninety percent of the solutes in grape juice are sugars, making these type of measuring methods a fairly accurate way of determining sugar levels of juice and calculating alcohol content of the wine. As the concentration of sugar in the grapes increases, the fermentation process produces higher levels of alcohols, which range from eight to as high as eighteen percent. Alcohol affects both the sensory attributes and stability of the wine [24, 25]. Therefore, sugar content of grapes is important in ascertaining harvest date and ultimately the quality of the finished product $[19,25]$.

\section{$\underline{\text { Winemaking }}$}

Though the art of making wine has remained the same for centuries the differences put into style are what keeps all wines a little unique. The condition of the grapes at the time they are picked is absolutely crucial to wine quality. The potential of a crop of grapes for making the best possible wine can be compromised by picking at the wrong time [5]. The decision to harvest grapes is dictated by maturity parameters that are guided by many factors. These include wine style, variety, and maturity criteria. Typically, these criteria include the course of when 
sugars accumulate, titratable acidity declines, $\mathrm{pH}$ rises, color, and phenolic compounds increase. It would be highly desirable to have all these parameters in ideal balance, but this is difficult to achieve since these parameters are influenced by many different factors [11].

Once grape maturity has arrived the harvest begins. It is imperative that crushing and destemming be done immediately in order to advert spoilage. This is done by machine and the object of de-stemming and crushing is to remove the stem and gently break the berry skin. The crushed fruit consisting of the pulp, skin, and seed is called must. It is transferred to a container and about $30-50 \mathrm{ppm}$ of $\mathrm{SO} 2$ is added. The purpose of the $\mathrm{SO} 2$ addition is to prevent development of unwanted microbes such as indigenous yeast and harmful bacteria [11]. Standard information suggests these steps be done in the following order: crush, add SO2, wait 12 hours, add yeast, ferment to 0 brix and press [12]. This order is a standard practice and many manipulations to this order can be done, which can result in more interesting wines which define the winemaker style. 


\section{Chapter 2}

\section{Materials and Methods}

\section{$\underline{\text { Variable Yield Trial Site Description }}$}

The variable yield trial site was conducted at Bien Nacido Vineyards, Santa Maria, California. The vineyard is located on Santa Maria Mesa road, approximately 10 miles east of Highway 101. The soil type at the trial site is a gravely/sandy loam. Grapevines were first planted in this block in 1973, and then tore out in 1992. The Pinot Noir clone 2A on 101-14 rootstock was then planted in 1994.

Rows are planted in a north-south direction. The vines are spaced 4 feet by 8 feet (vine $x$ row spacing), drip irrigated, and cane pruned. The canes are tied onto a vsp (vertical shoot positioning) trellis system. The training wire is 34 inches from the ground with four moveable catch wires above it.

\section{Experimental Design}

The trial consisted of 8 treatments and a control. The treatments and control totaled 258 vines. The 8 treatments encompassed 25 vines each and the control had 50 vines. A buffer vine separated the eight treatments and the control. The test plot consisted of 9 rows with the first containing 18 vines, second 21 vines, third 24 vines, fourth 27 vines, fifth 29 vines, sixth 31 vines, seventh 32 vines, eighth 37 vines, and the ninth had 38 vines. The irrigation and fertilizer program was kept consistent throughout the block with no variation. Removal of leaves was standardized across all 8 treatments and the control. The east side was striped of most leaves around the fruiting zone for sunlight exposure and the west side was moderately removed to encourage air flow. The leaf removal was performed on 6-13-02 and 6-16-03. Suckering 
(removal of non-fruitful shoots) was performed on 6-14-02 and 6-17-03, on the entire block. Mechanical hedging of the canopy throughout the block was performed once on 8-14-02 and 716-03.

\section{Crop Load Adjustments}

The historical data taken from Bien Nacido Vineyards on cluster weights showed an average of .25 pounds per cluster in pinot noir. In order to forecast the possible cropping levels this data was used in collaboration of cluster counts. The first 4 treatments were early fruit drops at post-bloom on 6-23-02 and 6-23-03. The first treatment was stripped of excess clusters, leaving seven for a possible crop load of 1 ton per acre. The second treatment left 14 for a possible 2 tons/acre, the third 21 clusters for a possible 3 tons/acre, and the fourth 28 clusters for a possible 4 tons/acre. The second 4 treatments were fruit drops on 8-30-02 and 8-17-03, at 75\% veraison (fruit becomes soft with the red varieties changing color) with the greenest of the fruit being discarded first. Again the first treatment left 7 clusters behind, second 14 clusters, third 21 clusters, and the fourth 28 clusters. The control was left alone, with no fruit removed.

\section{Caliper Readings}

A caliper is a tool used to measure small objects in either millimeters or inches. Caliper readings began on 7-9-02 and 7-18-03, with only the post-bloom treatments and control recorded. The readings were performed once a week with a random sampling method for choosing the clusters. Each cluster had 3 berries measured, one berry from the top, middle, and bottom of cluster. A total of 17 clusters were measured from each treatment. The 17 clusters yielded 51 berry readings. 
Fruit was dropped on 8-30-02 and 8-17-03, from the remaining 4 treatments at $75 \%$ veraison. Caliper readings began on 9-6-02 and 8-22-03, for the 4 treatments of $75 \%$ veraison. The eight treatments and the control were now being recorded weekly with 51 berry samples taken from each. Digital pictures were also taken in conjunction with the caliper readings on a weekly basis (Olympus, Camedia model \#D-360L). The caliper readings continued until harvest on 10-12-02 and 9-29-03.

\section{$\underline{\text { Harvest }}$}

Harvest commenced on 10-12-02 and 9-29-03, with all eight treatments and the control being harvested. Each vine was harvested separately, with the clusters being counted and weighed. This made it possible to figure out the average cluster weight for each vine within the eight treatments and the control. Each treatment and the control was crushed and destemed separately so that an accurate measurement of sugar and acid content could be recorded. The sugar content was recorded in degree brix using a handheld temperature compensating refractometer (Atago, model ATC-1E). An Orion analog pH meter, model 301, was used in conjunction with $0.10 \underline{\mathrm{N}}$ sodium hydroxide to measure the total acidity of each treatment.

\section{$\underline{\text { Wine Process }}$}

The eight treatments were combined into four. The post-bloom fruit drop was combined with the $75 \%$ veraison fruit drop, keeping the 7 cluster, 14 cluster, 21 cluster, and the 28 cluster samples separate. This yielded four cropping level treatments and also the control. The sugar content and total titratable acidity was again recorded in the same manner. The treatments along with the control were acidulated to a total titratable acidity of 7.5 grams per liter, or $.75 \%$, with tartaric acid. The acidity needed to be consistent for all the treatments since extractable phenols 
are very dependant upon the acid content (ETS Laboratories). Potassium meta-bi-sulfate (sulfur) was added at a rate of 30 parts per million, $.23 \mathrm{~g} / \mathrm{gallon}$, to kill off the wild strains of yeast that may be present in the must. Enoferm RA17 Burgundy isolate yeast was added at a rate of 2 grams per liter of must. Fermentation was performed in 115 liter Sterilite plastic storage totes with the cap being punched down twice a day. The must was measured for sugar content using a hydrometer until 0\% sugar remained (dry). The must was then pressed using a 15-gallon basket press. The wine then was placed in 5-gallon glass carboys. Wine samples from the 4 treatments and the control were taken out of the glass carboys on 2-5-03 and 2-10-04, then sent to ETS Laboratories in Saint Helena to perform a red wine phenolic report. Lino Bozzano of Tantara Winery was used as a consultant throughout the wine process.

\section{Pruning}

Pruning first took place on 1-15-02, 1-24-03, and 2-3-04. The first pruning job was a double cane per vine with no renewal spurs left. The canes were each left approximately 4 feet long causing them to overlap the vine next to it. This brought the total fruiting length of each vine to approximately 8 feet long. The second and third pruning job on 1-24-03 and 2-3-04, was done exactly in the same manner as the first with the two 4 feet long canes left per vine. The shoots from each vine were counted and then weighed on a temperature compensated field scale (CCI Scale Company) with the data recorded.

\section{$\underline{\text { Statistical Analysis }}$}

All data were analyzed using Minitab version 15. Results were determined using an appropriate General Linear Model. All analyses were performed at the 0.05 level. 


\section{Chapter 3}

\section{Results and Discussion}

Titratable Acidity (TA) was significantly different between the 2002 and 2003 growing seasons ( $\mathrm{p}=0.017$ ) with TA lower in 2003 than 2002. As mentioned in Chapter 1, titratable acidity measures the undissociated forms of acids, besides the concentration of free hydrogen ions. The undissociated forms of acids include organic acids, volatile acids, amino acids, and inorganic acids such as phosphoric acids [25]. This result is most likely due to the growing conditions of the two separate years. Year 2003 contained less chill hours, rain fall, and was harvested two weeks earlier than 2002 [Figure 3, Table 1]. There was no significant difference in titratable acidity between the early and late fruit thinning $(\mathrm{p}=0.694)$. These results indicate that the post bloom drop compared to seventy five percent veraison drop only slightly affected TA. There was also no significant difference between the tonnage treatments $(\mathrm{p}=0.157)$. The one ton, two ton, three ton, and four ton cropping levels also had minimal effect on TA. To summarize TA for both 2002 and 2003, we find the significant difference was by year and most likely caused by climatic conditions not treatments.

There was no significant difference with regards to $\mathrm{pH}$ between the 2002 and 2003 growing seasons $(\mathrm{p}=0.888)$. The early versus late fruit thinning resulted in no significant difference ( $\mathrm{p}=0.576$ ) with regards to $\mathrm{pH}$. The tonnage treatments also resulted in no significant difference ( $\mathrm{p}=0.316)$ with regards to $\mathrm{pH}$. No statistically significant change in $\mathrm{pH}$ was discovered in either year, timing of thinning, or cropping level. This was not the result found by Price et al., where reduced crop levels increased $\mathrm{pH}$ [23]. We would suspect the $\mathrm{pH}$ to have been significantly higher in reduced cropping levels. Table 1 exhibits that in 2002 the two ton early 
level was highest and in 2003 the four ton late was highest. We must conclude that $\mathrm{pH}$ was not a significant factor in this study.

Total Soluble Solids (Brix) analysis showed a significant difference between the early versus late fruit thinning ( $\mathrm{p}=0.033$ ) with the late fruit thinning resulting in a higher brix. Seventy five percent veraison thinning (late drop) as opposed to post set thinning (early drop) shows that there is an advanced amount of maturation that occurs, with concern to sugar content. This is most likely due to the added stress placed on the vine to ripen the added crop load (29). Another factor that must be taken into account is that the greener/less ripe fruit was noticeable at the time of the late drop due to the change in color. Less advanced fruit was dropped first in order to create a more uniform harvest. This selective fruit drop most likely caused the higher brix as opposed to the lower brix found in the early fruit drop. There was no significant difference between the tonnage treatments $(\mathrm{p}=0.064)$ with regards to brix. According to Bravado et al., high crop loads delayed the date of harvest throughout their five year trial [7]. Although not statistically significant according to appendix $\mathrm{O}$, we find a trend that exhibits a delay in ripening for lower tonnage levels [table 1]. There was no significant difference between the 2002 and 2003 growing seasons $(\mathrm{p}=0.934)$ with regards to brix. We can conclude that late fruit thinning ascertained a higher degree of brix most likely caused by two factors. First, the trial was conducted in a high vigour location which caused the vine to naturally be vegetative in growth, by leaving the fruit on the vine and dropping later caused a certain amount of added stress producing a more balanced vine causing the vine to ripen earlier. Second, the late fruit drop gave us the advantage of selecting (visually) more uniform clusters to drop, due to the change in color known as veraison [30]. 
There was not a statistically significant effect of drop level on the mean caliper size in $2002(\mathrm{p}=0.338)$. There is a statistically significant effect of drop date (early vs. late) on the mean caliper size in $2002(\mathrm{p}=0.22)$. When the drop is early, we estimate the mean caliper size to be 0.0114 larger than when the drop is later. This result is the mean caliper size across all eight treatments, which suggests that in general dropping early will usually leave larger berries. Larger berries can have a negative impact on phenolics, which can produce a less attractive wine do to less skin contact during the fermentation process [21]. According to Bravdo et al., increased berry weight/size did not improve wine quality, therefore an early fruit drop would not be advantageous to wine quality [7]. There is a statistically significant interaction between drop level and drop date in 2002 ( $\mathrm{p}=0.000)$. In particular, with a 3-ton drop level, an early drop produces grapes that are 0.0250 smaller than we would expect in the additive model and a late drop produces grapes that are 0.0250 larger than we would expect with the additive model. Compared to the no drop as a control group. This would suggest that the early three ton drop level would have a positive impact on phenolic extraction with smaller berries contributing more skin contact during fermentation when compared to the late three ton drop level [21]. There is not a statistically significant difference with the location of the berries within the cluster, top/middle/bottom in 2002 ( $\mathrm{p}=0.207$ ). To summarize the caliper measurements in 2002, when we compare drop date to the mean caliper size (berry size) we find that a late drop produces the smallest berries, on average, in a vigorous site. When we compare drop level to drop date, we find that at the three ton level, an early drop would be more advantageous to wine phenolics as opposed to a three ton late drop.

There is a statistically significant effect of drop level on the mean caliper size in 2003 ( $\mathrm{p}=0.000$ ). As opposed to 2002, this suggests that tonnage levels do impact the size of the 
berries. There is not a noticeable difference between a four ton drop and no drop at all, but three, two, and one ton drop levels produce larger grapes than no drop. This suggests that dropping time/date set aside, lower cropping levels produce larger grapes. According to Price et al., reducing crop levels led to a higher berry weight/size [23]. There is a statistically significant effect of drop date on the mean caliper size in $2003(\mathrm{p}=0.000)$. When the drop is early, we estimate the mean caliper size to be 0.0327 larger than when the drop is later. Therefore, 2003 suggests, as does 2002, that dropping early and lower tonnage levels will produce larger grapes. There is a statistically significant interaction between drop level and drop date in 2003 $(\mathrm{p}=0.000)$. In particular, with a three ton drop level, and early drop produces grapes that are 0.0440 larger than we would expect in the additive model and a late drop produces grapes that are 0.0440 smaller than we would expect with the additive model. This result contradicts what we find in the 2002 growing season. 2002 produced smaller berries with the three ton early drop as opposed to the late drop. With a four ton drop level, an early drop produces grapes that are 0.0445 smaller than we would expect with the additive model and a late drop produces grapes that are 0.0445 larger than we would expect with the additive model. Compared to no drop as a control group. There is not a statistically significant difference with the location of the berries within the cluster, top/middle/bottom in 2003 ( $\mathrm{p}=0.315$ ). To summarize the caliper measurements in 2003, when we compare drop date to the mean caliper size (berry size) we find that a late drop produces the smallest berries, on average, in a vigorous site. This is also the conclusion in 2002 which strengthens the argument that a late fruit drop is most advantageous to wine quality in a vigorous site. We can also conclude that the interaction in 2003 between drop level and drop date at the three ton level suggests that an early drop would produce larger grapes, contradicting the findings of 2002. 
There is a statistically significant relationship between drop level and average cluster weight in $2002(\mathrm{p}<.001)$. A post-hoc comparison of treatment group means was performed via the Tukey-Kramer procedure and those results indicate that on average, a one ton level has significantly higher oz/cluster/vine than the 2 ton level $(\mathrm{p}<.001)$, three ton level $(\mathrm{p}<.001)$, four ton level $(\mathrm{p}<.001)$ levels. No other differences were observed. There is also a statistically significant effect of the time of fruit drop on the average cluster weight in 2002 ( $\mathrm{p}=.001)$. Early has higher weights than late, on average. There is a statistically significant interaction between drop time and drop level $(\mathrm{p}<.001)$. At the 1,2, and 3 ton levels there is not a significant difference between the mean cluster weights. At the four ton level, early fruit drop has a significantly higher cluster weight. To summarize the cluster weights in 2002 , with small clusters producing the best possible wine quality, then we must ascertain that the one ton level produces the heaviest clusters thus the least attractive. According to Price et al., reducing crop level led to slightly less yield per vine, but higher cluster weights, berries per cluster, and berry weights [23]. The one ton level produced an actual .96 tons/acre on the early drop and 1.01 tons/acre on the late drop [table 1]. We can also conclude that on average, the early fruit drop had heavier clusters. If we wanted to produce more tons to the acre and still have smaller clusters, then we find that at the four ton level with a late drop had the greatest impact producing small clusters. The early four ton level produced an actual 3.39 tons to the acre and late four ton level produced 2.53 tons to the acre [table 1]. In conclusion, the 2002 growing season produced the best quality (smallest) clusters on average with the late fruit drop.

There is a statistically significant relationship between drop level and the average cluster weight in $2003(\mathrm{p}<.001)$. A post-hoc comparison of treatment group means was performed via the Tukey-Kramer procedure and those results indicate that on average, a two ton level has 
higher mean cluster weights than the control $(\mathrm{p}<.014)$. The three ton level has higher mean cluster weights than the four ton level $(\mathrm{p}=.019)$, and the control $(\mathrm{p}=.001)$. No other statistically significant differences were observed. There is not a statistically significant relationship between drop time and average cluster weight in $2003(\mathrm{p}=.121)$. There is a statistically significant interaction between drop time and drop level. At the one ton level, the early drop has a lower mean weight than we would have expected from an additive model $(\mathrm{p}=.005)$. No other statistically significant interactions were observed. To summarize the cluster weights in 2003, we find that the three ton level produced higher mean cluster weights on average than the four ton level $(\mathrm{p}=.019)$, and the control $(\mathrm{p}=.001)$. The three ton level produced an actual 2.57 tons to the acre on the early drop and 2.1 tons to the acre on the late drop [table 1]. The one ton level with early drop had a lower mean weight than we would have expected from an additive model, which opposes our findings in 2002. In conclusion the 2003 growing season opposed the results found from the 2002 growing season.

We are using the square root of the 2002 pruning weight (in ounces) as the response variable for our analyses. This is because if we had used the actual pruning weights we would have reason to believe the ANOVA assumption of normality to be invalid. With this change in the response variable, there is a statistically significant relationship between drop level and average shoot weight in $2002(\mathrm{p}<.001)$. The average shoot weight is significantly higher at the one ton level than it is at the three ton level $(\mathrm{p}<.001)$, four ton level $(\mathrm{p}=.006)$, and no fruit drop $(p<.001)$ levels. No other significant effects were found. There is also a significant relationship between drop time and average shoot weight in 2002 ( $\mathrm{p}<.001)$ with the late fruit drop being followed by a lower shoot weight. There is not a significant interaction between drop level and drop time in $2002(\mathrm{p}=.053)$. This means that there effect of drop level on the shoot weights does 
not significantly differ by drop time. To summarize the shoot weights in 2002 , we find that all treatments would be classified as high vigour according to Robinson and Smart [27]. The yield to pruning weight ratio model used by Robinson and Smart states that the yield to pruning weight ratio is considered high vigour at $<3$ [27]. The ratio of yield to pruning weight for 2002 gives us a range of .39 to 1.78 , concluding a high vigour site [table 3].

We are using the square root of the 2003 pruning weight (in ounces) as the response variable for our analyses. This is because if we had used the actual pruning weights we would have reason to believe the ANOVA assumption of normality to be invalid. With this change in the response variable, there is a statistically significant relationship between drop level and average shoot weight in 2003 ( $\mathrm{p}=.004)$. The average shoot weight is significantly lower at the three ton level than it is at the one ton level $(\mathrm{p}=.041)$ and at the four ton level $(\mathrm{p}=.029)$. No other significant effects were found. There is a significant relationship between drop time and average shoot weight in 2003 ( $\mathrm{p}=.049)$ with the late drop resulting in lower pruning weights. There is not a significant interaction between drop level and drop time in $2003(\mathrm{p}=.344)$. This means that there effect of drop level on the shoot weights does not significantly differ by drop time. To summarize the shoot weights for 2003, we find that all treatments would be classified as high vigour according to Robinson and Smart [27]. The yield to pruning weight ratio model used by Robinson and Smart states that the yield to pruning weight ratio is considered high vigour at $<3$ [27]. The ratio of yield to pruning weight for 2003 gives us a range of .32 to 1.36 , concluding a high vigour site [table 4]. 2003 expressed a significantly lower shoot weight at the three ton level than at the one ton level $(\mathrm{p}=0.41)$, and at the four ton level $(\mathrm{p}=.029)$, which strengthens the findings in 2002 that a lower tonnage level increased growth vigour. Both 2002 and 2003 found a significant relationship between drop time and average shoot weight, 2002 ( $\mathrm{p}<.001)$, and 2003 
$(\mathrm{p}=.049)$. This significant relationship expressed that an early drop increased the shoot weight which contributed only more vigour to an already high vigour site.

The mean level of gallic acid differs significantly by year $(\mathrm{F}=28.63, \mathrm{p}=0.006)$, but not across our five treatments $(\mathrm{F}=1.48, \mathrm{p}=0.357)$. The main sources of gallic acid in wine are grape seeds and oak cooperage. Since no oak was used on the wine, prior to laboratory analysis, we can assume the gallic acid content is derived from the seeds. Sourcing that seeds are the contributing factor here to a significant difference between years with concern to gallic acid, one must assume the earlier harvest in 2003 as a possible explanation.

The mean level of catechin differs significantly by year $(\mathrm{F}=53.45, \mathrm{p}=.002)$, but not across our five treatments $(\mathrm{F}=1.45, \mathrm{p}=.363)$. Catechin is known as a flavonol, flavon-3-ols, or procyanidins. Catechin compounds are found in high concentrations in seeds, stems, and may be found in the skins of immature grapes [8]. As grapes mature the seed coat is formed making seed phenolics increasingly difficult to extract. This leads to the assumption that the difference in maturity varied significantly by year leaving a higher content of the catechin flavonol in 2003.

The mean level of epicatechin differs significantly by year $(\mathrm{F}=30.91, \mathrm{p}=0.005)$, but not across our five treatments $(\mathrm{F}=2.16, \mathrm{p}=0.237)$. Epicatechin is known also as a flavonol, flavon-3ols, or procyanidins. Epicatechin compounds are found in high concentrations in seeds, stems, and may be found in the skins of immature grapes [8]. Once again, as grapes mature the seed coat is formed making seed phenolics increasingly difficult to extract. This leads to the assumption again that the difference in maturity varied significantly by year leaving a higher content of the epicatechin flavonol in 2003.

The mean level of polymeric phenols differs significantly by year $(\mathrm{F}=14.9672$, $\mathrm{p}=0.01801)$, but not across our five treatments $(\mathrm{F}=0.2352, \mathrm{p}=0.90506)$. Wine tannins are 
complex polymers that strongly affect wine flavor and color. Tannin includes phenolic polymers from grape seeds, skins, and stems as well as compounds formed or modified in wine.

Extraction of polymeric phenols (tannins) is driven by temperature, time, alcohol content and physical manipulations such as pumpovers [8]. The significant difference between years leads us to believe that the extraction process during the fermentation process could have been skewed since no temperature readings were reported for either year.

The mean level of caftaric acid does not differ significantly by year $(\mathrm{F}=3.4177$, $\mathrm{p}=0.1382)$, and not across our five treatments $(\mathrm{F}=2.96, \mathrm{p}=0.161)$.

The mean level of quercetin glycosides differs significantly by year $(F=11.59, p=0.027)$, but not differ across our five treatments $(\mathrm{F}=0.40, \mathrm{p}=0.802)$. Quercetin is a flavonol present in grape skins and stems as several different glycosides (compounds with attached sugars).

Quercetin accumulates in grape skins to protect against damage from ultra violet light. There are high concentrations of quercetin in sun exposed grape skins and in wine made from sun exposed grapes. Quercetin is readily extracted from grape skins during fermentation [8]. Quercetin glycosides are significantly different with a higher content found in the 2002 growing season. The practice of leafing was performed in the same manner both years, but was not measured. We must assume that more ultra violet light was exposed to the fruit in 2002 than in 2003, thus giving us a higher content of quercetin glycosides in 2002.

The mean level of quercetin aglycone does not differ significantly by year $(\mathrm{F}=2.67$, $\mathrm{p}=0.178)$, and does not differ across our five treatments $(\mathrm{F}=4.00, \mathrm{p}=0.104)$.

The mean level of malvidin glucoside does not differ significantly by year $(\mathrm{F}=0.0176$, $\mathrm{p}=0.9008)$, and does not differ across our five treatments $(\mathrm{F}=1.8992, \mathrm{p}=0.2748)$. 
The mean level of polymeric anthocyanins does not differ significantly by year $(\mathrm{F}=0.1546, \mathrm{p}=0.7142)$, and does not differ across our five treatments $(\mathrm{F}=0.1787, \mathrm{p}=0.9380)$.

The mean level of total anthocyanins does not differ significantly by year $(\mathrm{F}=0.1803$, $\mathrm{p}=0.6930)$, and does not differ across our five treatments $(\mathrm{F}=2.5213, \mathrm{p}=0.1961)$.

The mean level of monomeric anthocyanins does not differ significantly by year $(\mathrm{F}=0.1801, \mathrm{p}=0.6931)$, and does not differ across our five treatments $(\mathrm{F}=2.1831, \mathrm{p}=0.2341)$.

To summarize the phenolic analysis of 2002 and 2003, we find the only significant difference was found in five of the eleven phenolic compounds. The five significant differences differed only by year, and not across the five treatments. We must conclude that the differences found between 2002 and 2003 were most likely caused by two factors. First, the climatic conditions varied widely for both 2002 and 2003 [figure 3]. Secondly, we did not record or control the temperature of the fermentations in either year. Temperatures during fermentation can play an important role on the amount of phenolic extraction, with higher temperatures enhancing the extraction of phenols and color [21,31,32].

In conclusion, for pinot noir wine grapes, with high vine vigour, under similar trial conditions, it probably is unnecessary to perform cultural practices that decrease the tonnage level below the four ton treatment. Furthermore, early (post-bloom) thinning is not recommended, as berry size increased, sugars decreased and shoot weights increased, creating a detrimental vine balance. 


\section{Chapter 4}

\section{Recommendations for Further Study}

Recommendations for further study would narrow the experiment down to a reasonable amount of variables. I believe we took on too much for this study and some analyses may have suffered. As a vineyard manager, I believe we should have kept the vineyard practices separate from the wine making procedures. We should have used juice samples, sending the juice samples to ETS laboratories for analysis which would have minimized the problems incurred by producing the wine. The problems during the wine making process varied, but most stemmed from too small amount of juice which led to a multitude of problems, including stuck fermentations. My recommendation would be to send ETS laboratories a juice, berry, or cluster sample for a rapid phenolic panel which measures four components, catehin, polymeric anthocyanins, tannins, and total anthocyanins.

If we were to continue with the wine producing segment of this experiment then we should quadruple the treatment size at a minimum, so that we have a large enough sample base for the wine production, keeping the early and late drops separate along with the tonnage levels. A professional wine maker should oversee the production of winemaking with accurate notes taken on fermentation temperatures to ensure an even extraction of phenols and color from all treatments.

Caliper readings should also be in concert with berry counts at time of harvest. Berry counts per cluster would have given us a better estimate of berry weight. Shoot weights should also be measured along with diameter of shoots and number of nodes per shoot to give a more accurate account of vigour across our eight treatments. 
Finally, we should perform a statistical combined analysis of both years, 2002 and 2003. Our conclusions had conflicting results when comparing the 2002 and 2003 growing seasons in regards to berry size (caliper readings), cluster weights, and shoot weights. A combined analysis may offer some more refined conclusions. 
Figure 1. Degree hours accumulated from Adcon weather station for both 2002 and 2003 growing years.
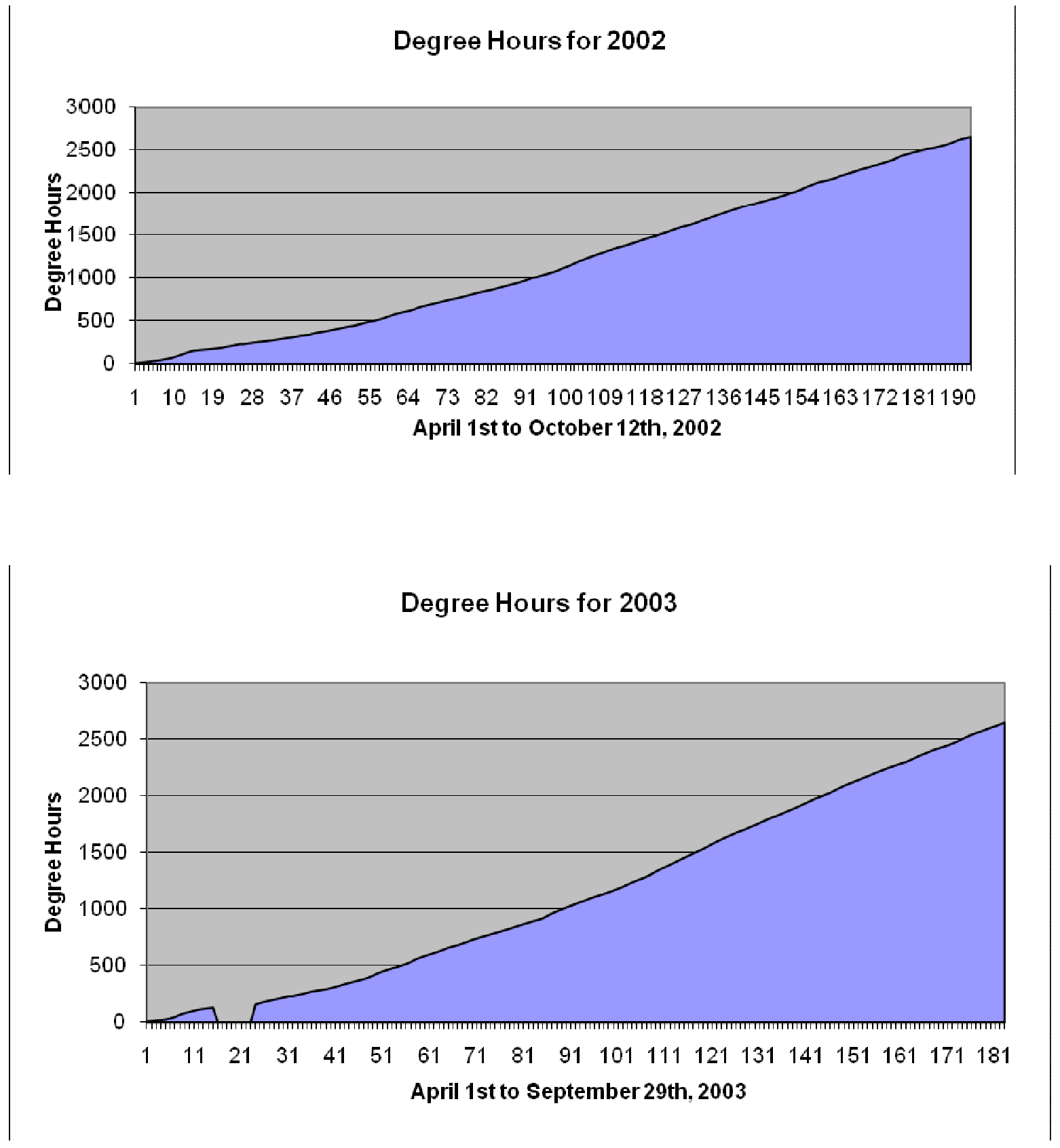
Figure 2. Chill hours accumulated from Adcon weather station for both 2002 and 2003 growing years.
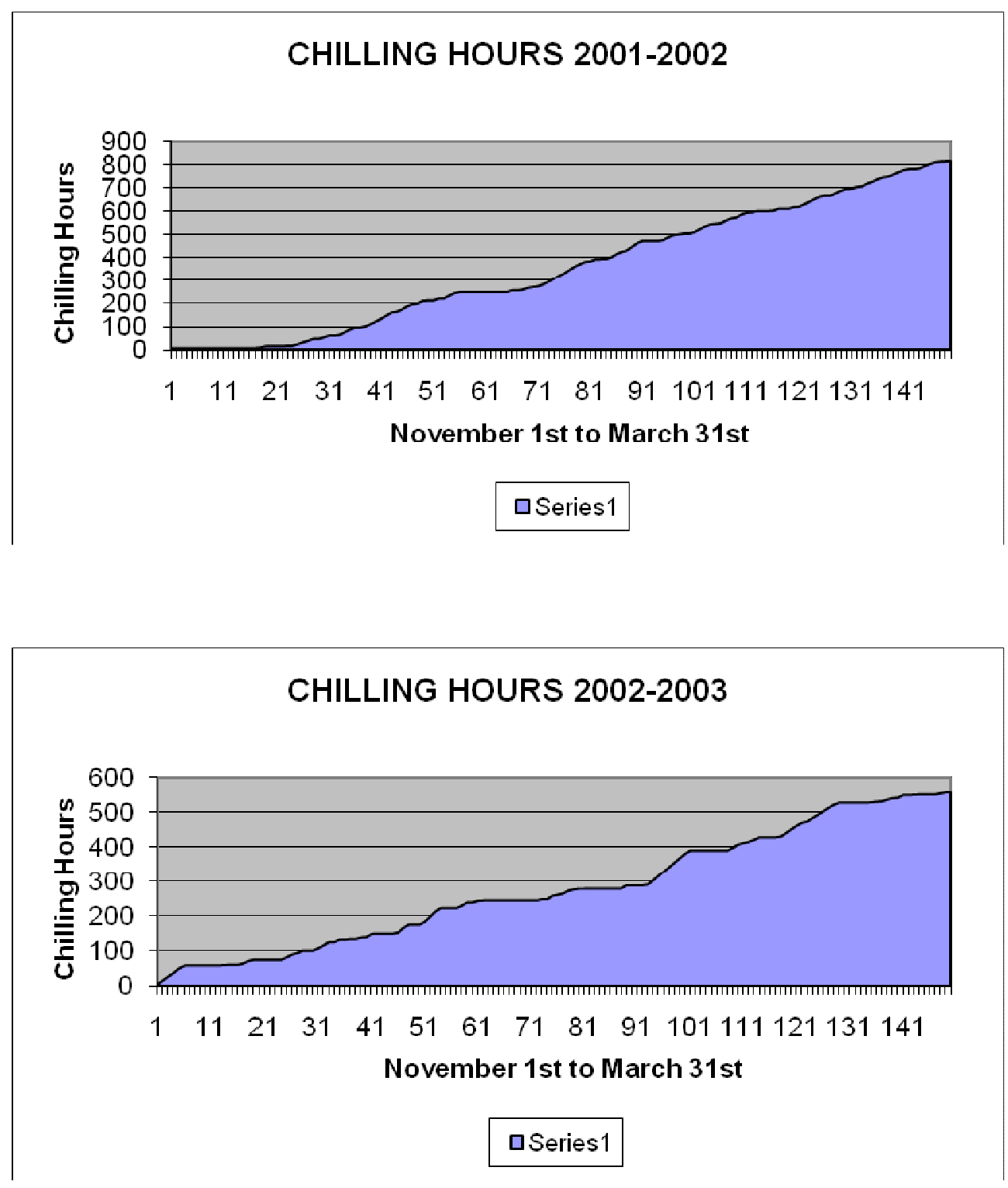
Figure 3. Weather data from Adcon weather station, 2002 \& 2003 side by side.

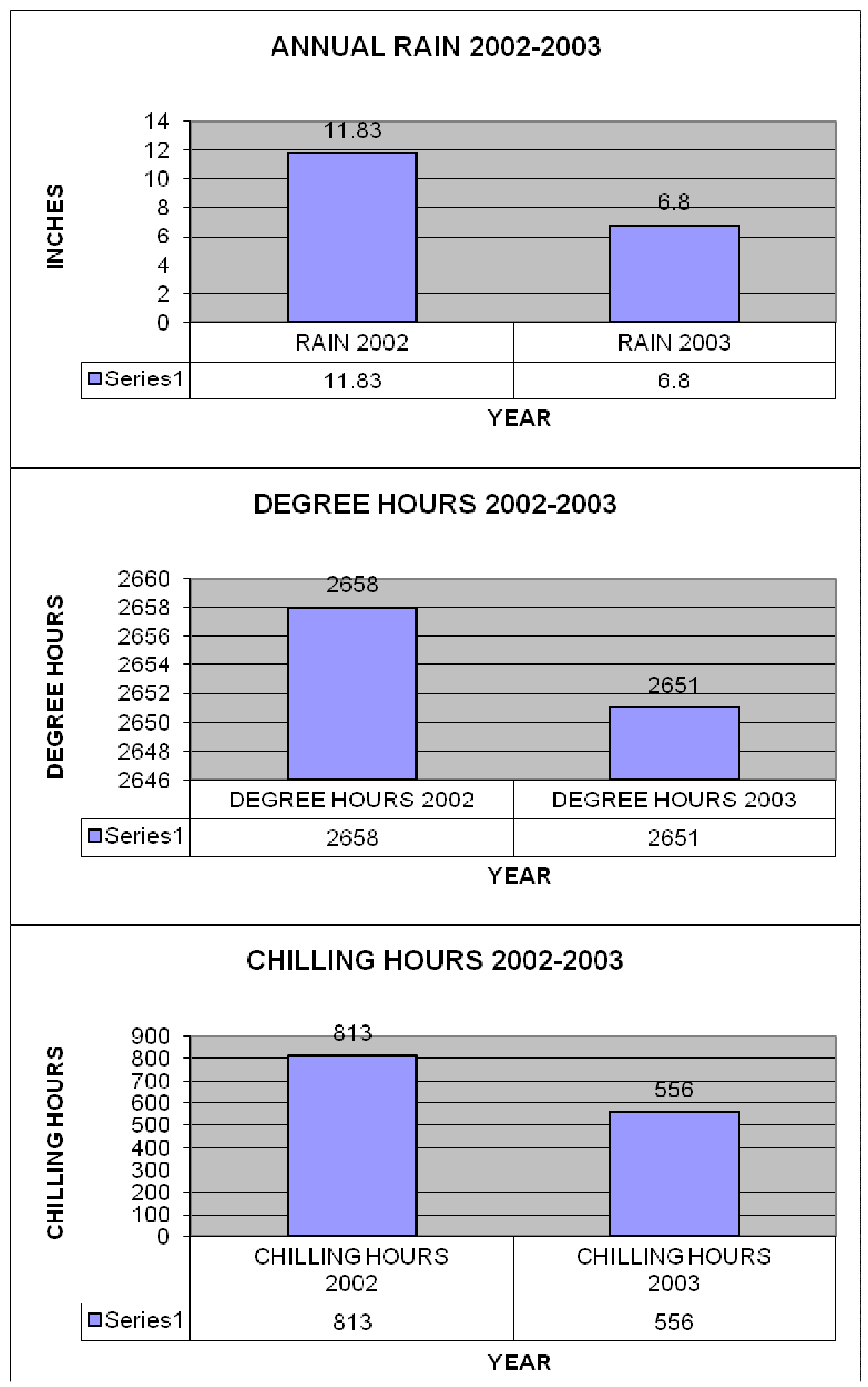


Figure 4. Photograph of Trial Site.

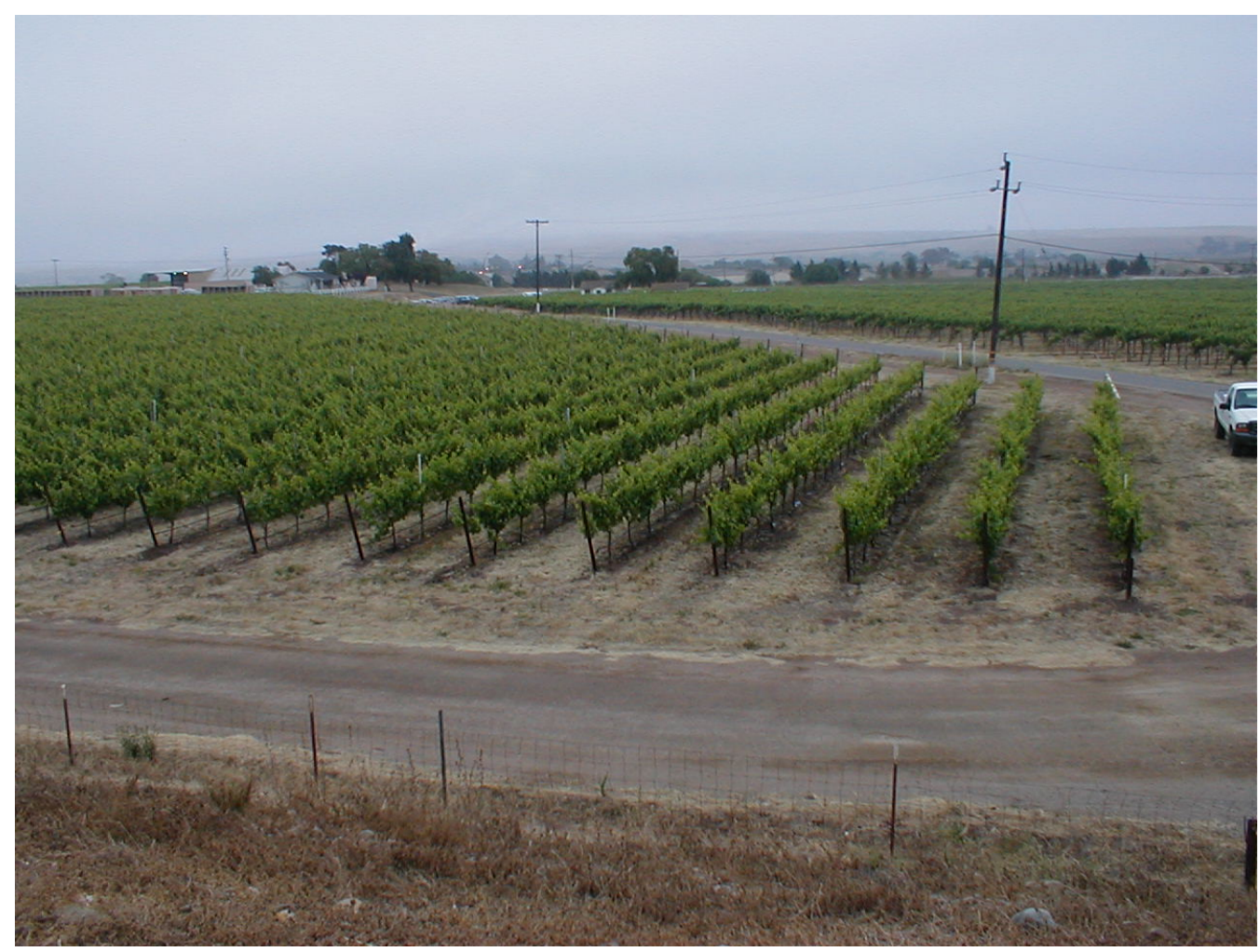

Photograph of two ton early drop treatment.

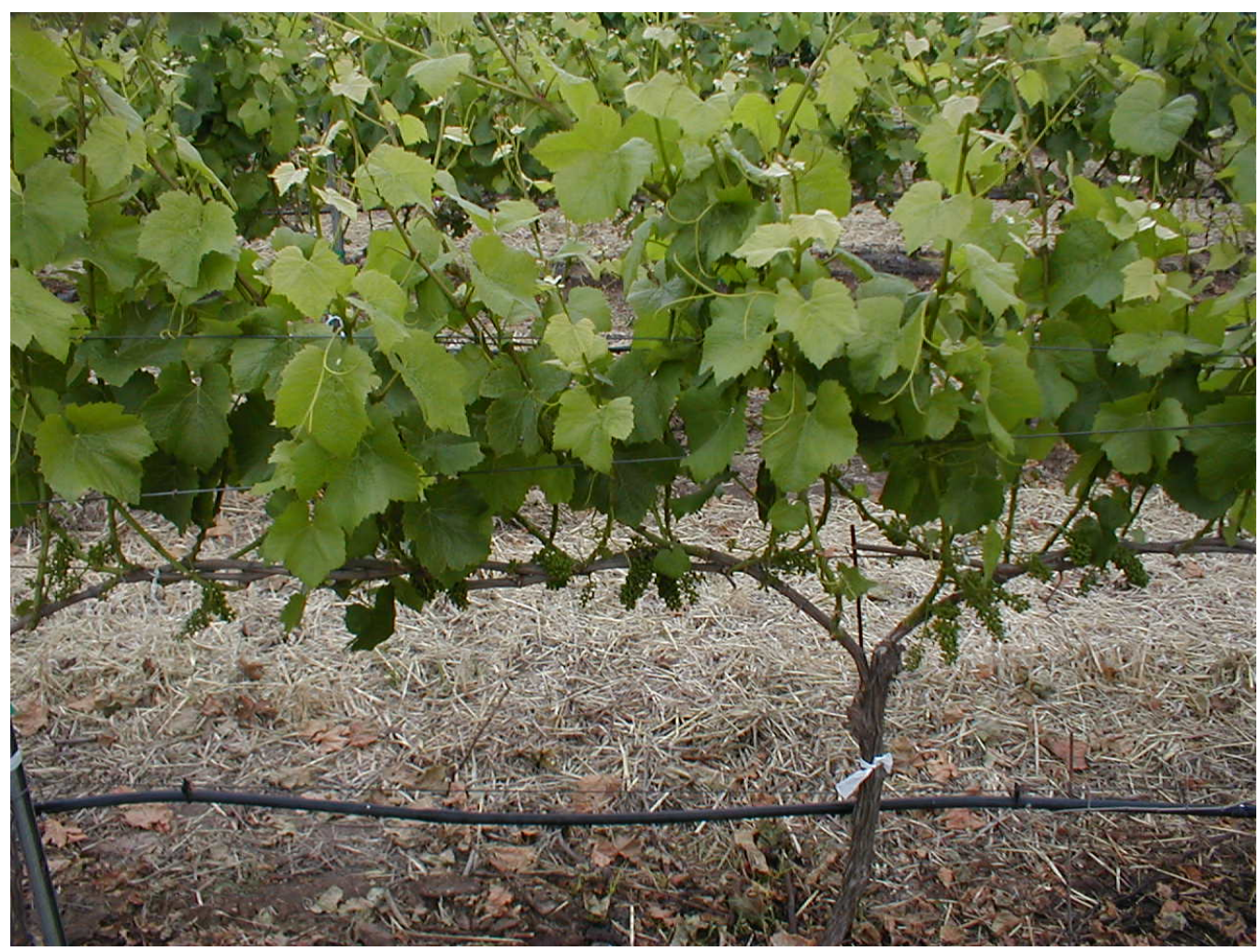


Figure 5. Photograph of one ton early drop treatment pre-veraison.

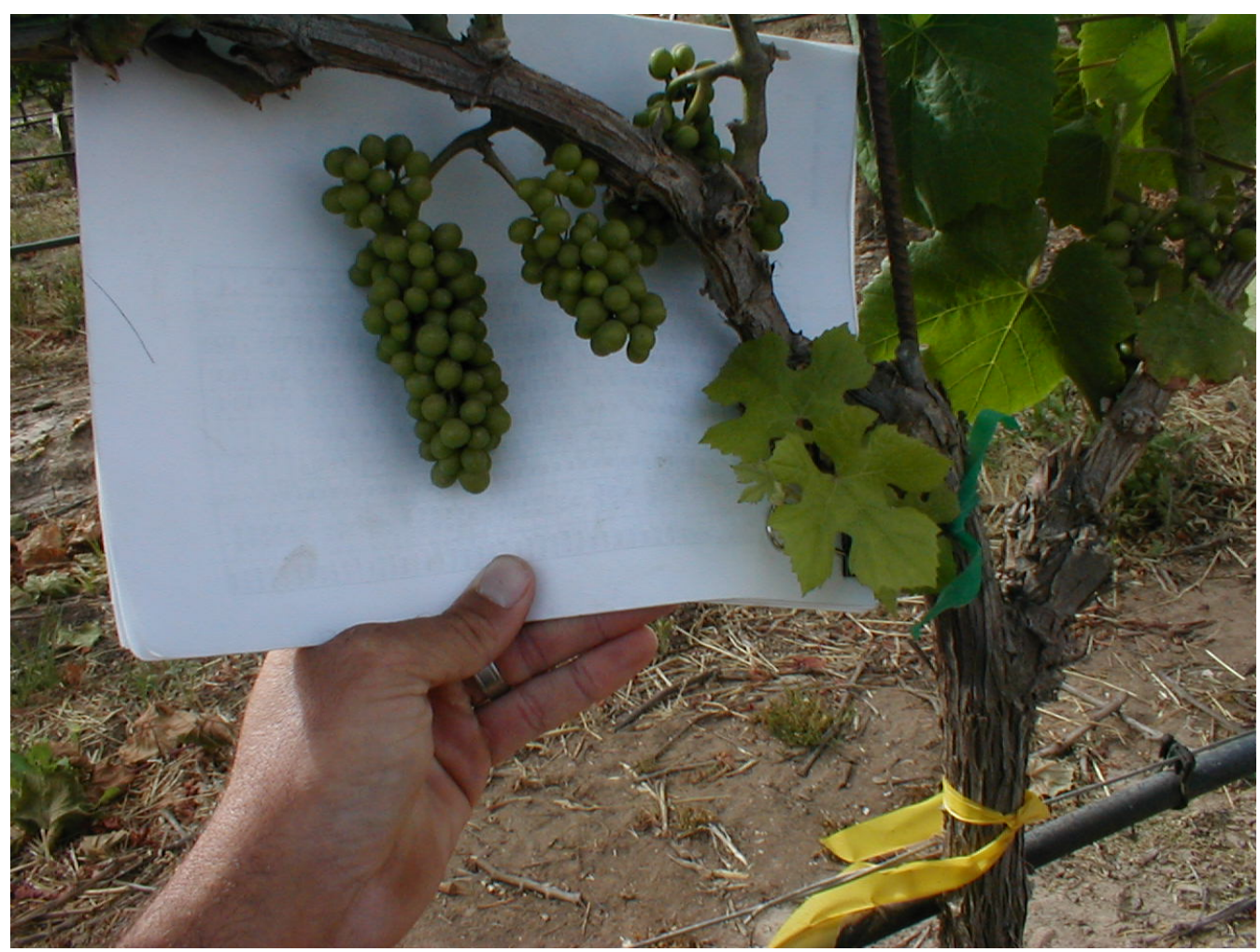

Photograph of five ton control treatment pre-veraison.

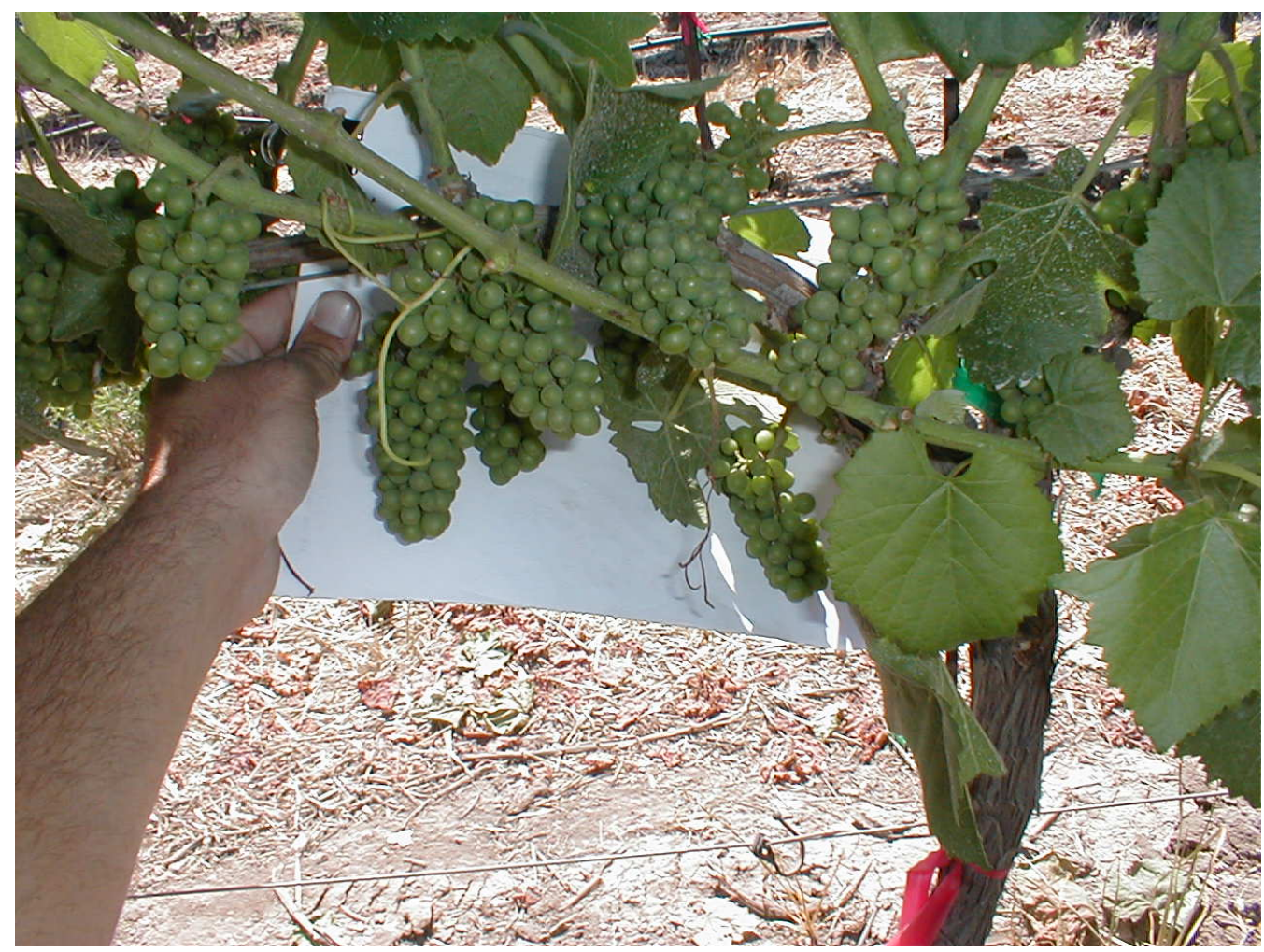


Figure 6. Photograph of pruned three ton treatment.

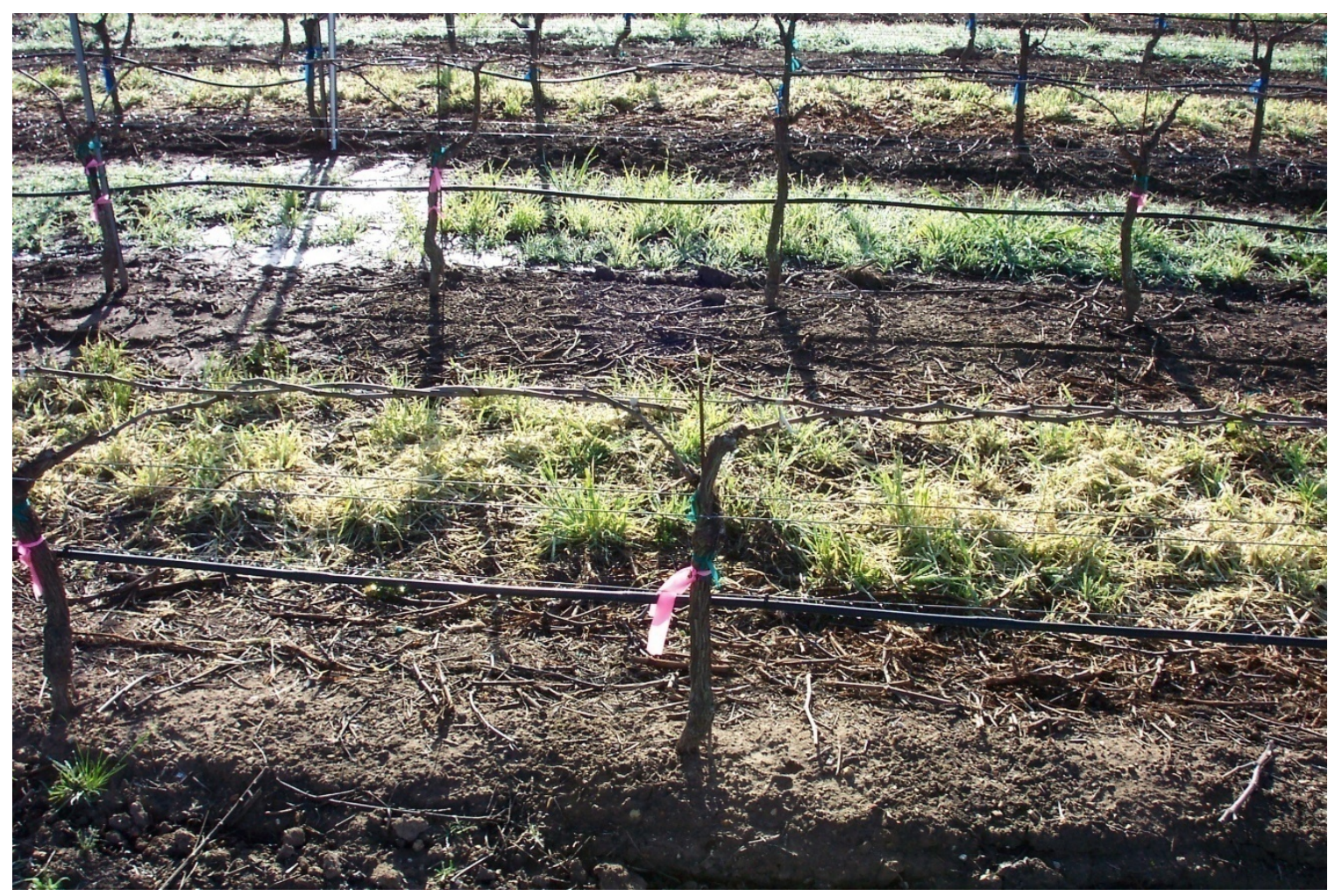

Photograph of pruned trial site.

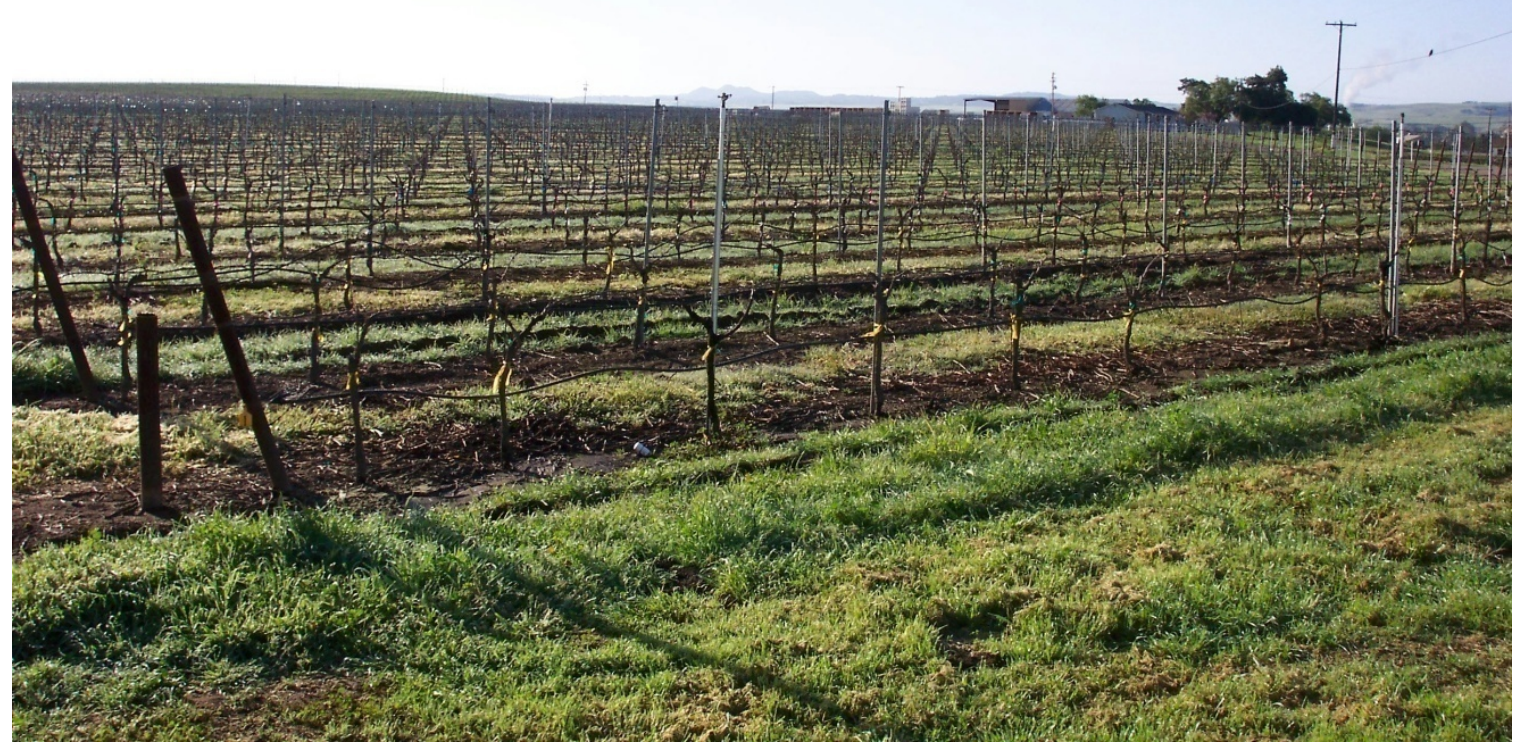


Figure 7. Map layout of trial site.

\begin{tabular}{|c|c|c|c|c|c|c|c|c|c|}
\hline \multicolumn{10}{|c|}{ Rows } \\
\hline Vines & 1 & $\underline{2}$ & $\underline{3}$ & $\underline{4}$ & $\underline{5}$ & $\underline{6}$ & $\underline{7}$ & 8 & 9 \\
\hline 1 & & & & & & & & $r$ & $r$ \\
\hline 2 & $y$ & yy & yy & ww & ww & $\mathrm{pp}$ & $\mathrm{pp}$ & $r$ & $r$ \\
\hline 3 & $y$ & yy & yy & $w w$ & ww & $\mathrm{pp}$ & $\mathrm{pp}$ & $r$ & $r$ \\
\hline 4 & $y$ & yy & yy & $w w$ & $w w$ & $\mathrm{pp}$ & $\mathrm{pp}$ & $r$ & $r$ \\
\hline 5 & $y$ & yy & yy & ww & ww & $\mathrm{pp}$ & $\mathrm{pp}$ & $r$ & $r$ \\
\hline 6 & $y$ & yy & yy & ww & $w w$ & $\mathrm{pp}$ & $\mathrm{pp}$ & $r$ & $r$ \\
\hline 7 & missing & missing & yy & ww & ww & $\mathrm{pp}$ & control & $r$ & $r$ \\
\hline 8 & $y$ & yy & yy & $w w$ & $w w$ & $\mathrm{pp}$ & $b$ & $r$ & $r$ \\
\hline 9 & $y$ & yy & yy & $w w$ & $w w$ & $\mathrm{pp}$ & $b$ & $r$ & $r$ \\
\hline 10 & $y$ & yy & yy & ww & ww & $\mathrm{pp}$ & $b$ & $r$ & $r$ \\
\hline 11 & $y$ & yy & yy & $w w$ & ww & $\mathrm{pp}$ & $b$ & $r$ & $r$ \\
\hline 12 & $y$ & yy & $y$ & ww & ww & $\mathrm{pp}$ & $b$ & $r$ & $r$ \\
\hline 13 & $\mathrm{y}$ & yy & yy & $w w$ & $w w$ & $\mathrm{pp}$ & $b$ & control & $r$ \\
\hline 14 & $y$ & yy & control & control & ww & $\mathrm{pp}$ & $b$ & $\mathrm{bb}$ & $r$ \\
\hline 15 & $y$ & yy & $w$ & $w$ & control & $\mathrm{pp}$ & $b$ & $\mathrm{bb}$ & $r$ \\
\hline 16 & $y$ & control & $w$ & $w$ & $p$ & $\mathrm{pp}$ & $b$ & bb & $r$ \\
\hline 17 & $y$ & $\mathrm{y}$ & w & w & $p$ & $\mathrm{pp}$ & $b$ & $\mathrm{bb}$ & $r$ \\
\hline 18 & $y$ & $y$ & $w$ & $w$ & $p$ & $\mathrm{pp}$ & $b$ & $\mathrm{bb}$ & $r$ \\
\hline 19 & $y$ & $y$ & w & w & $p$ & $\mathrm{pp}$ & $b$ & bb & missing \\
\hline 20 & $y$ & $y$ & $w$ & $w$ & $p$ & $\mathrm{pp}$ & $b$ & bb & $r$ \\
\hline 21 & & $y$ & $w$ & $w$ & $p$ & $\mathrm{pp}$ & $b$ & $\mathrm{bb}$ & $r$ \\
\hline 22 & & $y$ & w & w & $p$ & control & $b$ & $\mathrm{bb}$ & $r$ \\
\hline 23 & & $y$ & $w$ & $w$ & $p$ & $p$ & $b$ & $\mathrm{bb}$ & $r$ \\
\hline 24 & & & w & w & $p$ & $p$ & $b$ & $\mathrm{bb}$ & $r$ \\
\hline 25 & & & $w$ & $w$ & $p$ & $p$ & $b$ & $\mathrm{bb}$ & $r$ \\
\hline 26 & & & & $w$ & $p$ & $p$ & $b$ & $\mathrm{bb}$ & $r$ \\
\hline 27 & & & & w & $p$ & $p$ & $b$ & $\mathrm{bb}$ & $r$ \\
\hline 28 & & NORTH & & $w$ & $p$ & $p$ & $b$ & bb & $r$ \\
\hline 29 & WEST & + & EAST & & $p$ & $\mathrm{p}$ & $b$ & $\mathrm{bb}$ & $r$ \\
\hline 30 & & SOUTH & & & $p$ & $p$ & $b$ & $\mathrm{bb}$ & $r$ \\
\hline 31 & & & & & & $p$ & $b$ & $\mathrm{bb}$ & $r$ \\
\hline 32 & & \multirow{2}{*}{\multicolumn{3}{|c|}{$\begin{array}{l}Y=\text { Post-Bloom drop } / 1 \text { ton } \\
Y Y=75 \% \text { veraison drop } / 1 \text { ton }\end{array}$}} & & $p$ & $b$ & bb & $r$ \\
\hline 33 & & & & & & & control & bb & $r$ \\
\hline 34 & & \multicolumn{3}{|c|}{ W = Post-Bloom drop/2 ton } & & & missing & $\mathrm{bb}$ & $r$ \\
\hline 35 & & \multicolumn{3}{|c|}{ WW $=75 \%$ veraison drop $/ 2$ ton } & & & bb & $\mathrm{bb}$ & $r$ \\
\hline 36 & & \multicolumn{3}{|c|}{$\mathbf{P}=$ Post-Bloom drop $/ 3$ ton } & & & stunted & $\mathrm{bb}$ & $r$ \\
\hline 37 & & \multicolumn{3}{|c|}{ PP $=75 \%$ veraison drop $/ 3$ ton } & & & & $\mathrm{bb}$ & $r$ \\
\hline 38 & & \multicolumn{3}{|c|}{ B = Post-Bloom drop/4 ton } & & & & & $r$ \\
\hline 39 & & \multicolumn{3}{|c|}{ BB $=75 \%$ veraison drop $/ 4$ ton } & & & & & $r$ \\
\hline 40 & & \multicolumn{3}{|c|}{$\mathbf{R}=$ Control } & & & & & \\
\hline
\end{tabular}


Figure 8. 2002 growing season.

1-13-03 Trial site map containing number of canes pruned/vine.

\begin{tabular}{|c|c|c|c|c|c|c|c|c|c|}
\hline \multicolumn{2}{|r|}{$\underline{1}$} & \multirow[t]{2}{*}{$\underline{2}$} & \multirow[t]{2}{*}{$\underline{3}$} & \multirow[t]{2}{*}{$\underline{4}$} & \multirow[t]{2}{*}{$\underline{5}$} & \multirow[t]{2}{*}{$\underline{6}$} & $\underline{7}$ & \multirow{2}{*}{$\frac{\underline{8}}{r 15}$} & \multirow{2}{*}{$\mathrm{r} 20$} \\
\hline 1 & & & & & & & & & \\
\hline 2 & $\mathrm{y} 11$ & yy19 & yy24 & ww20 & ww17 & pp22 & pp20 & $\mathrm{r} 11$ & $\mathrm{r} 18$ \\
\hline 3 & $\mathrm{y} 17$ & yy22 & yy 19 & ww23 & ww25 & pp23 & pp19 & r21 & $\mathrm{r} 17$ \\
\hline 4 & $\mathrm{y} 15$ & yy21 & yy20 & ww17 & ww21 & pp14 & pp19 & r22 & r22 \\
\hline 5 & y24 & yy25 & yy30 & ww19 & ww18 & pp26 & pp19 & r23 & r22 \\
\hline 6 & y21 & yy23 & yy15 & ww22 & ww20 & pp20 & pp26 & $r 17$ & r22 \\
\hline 7 & missing & missing & yy20 & ww24 & ww13 & pp27 & control & $r 18$ & $r 19$ \\
\hline 8 & $\mathrm{y} 24$ & yy 17 & yy22 & ww19 & ww18 & pp25 & $\mathrm{b} 19$ & r23 & r23 \\
\hline 9 & y20 & yy15 & yy22 & ww23 & ww20 & pp22 & b20 & $r 16$ & r22 \\
\hline 10 & $\mathrm{y} 22$ & yy19 & yy22 & ww24 & ww23 & pp31 & $\mathrm{b} 25$ & $\mathrm{r} 18$ & $\mathrm{r} 16$ \\
\hline 11 & y25 & yy 15 & yy22 & ww17 & ww16 & pp20 & $\mathrm{b} 21$ & r20 & r20 \\
\hline 12 & y20 & yy25 & yy20 & ww20 & ww27 & pp24 & b17 & r25 & $r 13$ \\
\hline 13 & $\mathrm{y} 20$ & yy 19 & yy22 & ww19 & ww21 & pp21 & $\mathrm{b} 21$ & control & $\mathrm{r} 27$ \\
\hline 14 & y21 & yy21 & control & control & ww20 & pp16 & b13 & bb18 & r24 \\
\hline 15 & $\mathrm{y} 18$ & yy15 & w21 & w17 & control & pp22 & b18 & bb15 & $\mathrm{r} 17$ \\
\hline 16 & $\mathrm{y} 19$ & control & w18 & w20 & p21 & pp19 & $\mathrm{b} 21$ & bb31 & $\mathrm{r} 28$ \\
\hline 17 & y25 & $\mathrm{y} 18$ & w23 & w22 & p21 & pp24 & b26 & bb26 & r22 \\
\hline 18 & $\mathrm{y} 17$ & $\mathrm{y} 22$ & w19 & w24 & p20 & pp26 & b18 & bb18 & $\mathrm{r} 28$ \\
\hline 19 & $\mathrm{y} 19$ & $\mathrm{y} 17$ & w24 & w24 & p19 & pp20 & b26 & bb26 & missing \\
\hline 20 & y27 & y29 & w21 & w24 & p22 & pp20 & b16 & bb28 & r27 \\
\hline 21 & & $\mathrm{y} 19$ & w20 & w21 & p21 & pp27 & b17 & bb22 & r21 \\
\hline 22 & & $\mathrm{y} 18$ & w23 & w27 & p22 & control & b23 & bb20 & r22 \\
\hline 23 & & $y 22$ & w20 & w20 & p23 & p24 & b26 & bb21 & $\mathrm{r} 18$ \\
\hline 24 & & & w19 & w19 & p23 & p25 & b24 & bb24 & r22 \\
\hline 25 & & & w23 & w21 & p22 & p27 & b20 & bb25 & r22 \\
\hline 26 & & & & w20 & p17 & p25 & b20 & bb23 & $\mathrm{r} 19$ \\
\hline 27 & & & & w19 & p23 & p23 & b21 & bb25 & $\mathrm{r} 22$ \\
\hline 28 & & & & w21 & p22 & p24 & b21 & bb25 & $\mathrm{r} 20$ \\
\hline 29 & & & & & p15 & p22 & $\mathrm{b} 21$ & bb20 & r24 \\
\hline 30 & & & & & p21 & p18 & b20 & bb26 & r25 \\
\hline 31 & & & & & & p18 & b19 & bb23 & r25 \\
\hline 32 & & & NORTH & & & $\mathrm{p} 26$ & b31 & bb22 & r23 \\
\hline 33 & & WEST & + & EAST & & & control & bb24 & r24 \\
\hline 34 & & & SOUTH & & & & missing & bb28 & r29 \\
\hline 35 & & & & & & & bb28 & bb23 & r26 \\
\hline 36 & & & & & & & stunted & bb26 & r21 \\
\hline 37 & & & & & & & & bb27 & $\mathrm{r} 19$ \\
\hline 38 & & & & & & & & & r24 \\
\hline 39 & & & & & & & & & r22 \\
\hline
\end{tabular}


Figure 9. 2002 growing season.

1-13-03 Trial site map containing ounces of prunings/vine.

\begin{tabular}{|c|c|c|c|c|c|c|c|c|c|}
\hline & $\underline{1}$ & $\underline{2}$ & $\underline{3}$ & $\underline{4}$ & $\underline{5}$ & $\underline{6}$ & $\underline{7}$ & $\underline{8}$ & $\underline{9}$ \\
\hline 1 & & & & & & & & r36 & r36 \\
\hline 2 & y39 & yy45 & yy51 & ww60 & ww52 & pp56 & pp46 & $\mathrm{r} 14$ & r26 \\
\hline 3 & y36 & yy46 & yy54 & ww64 & ww64 & pp43 & pp44 & r57 & r26 \\
\hline 4 & $y 75$ & уу36 & yy57 & ww25 & ww45 & pp15 & pp41 & r58 & r54 \\
\hline 5 & $y 63$ & yy62 & yy58 & ww44 & ww22 & pp36 & pp25 & $\mathrm{r} 41$ & r33 \\
\hline 6 & y91 & yy43 & yy36 & ww43 & ww34 & pp48 & pp69 & r30 & r39 \\
\hline 7 & missing & missing & yy43 & ww65 & ww46 & pp34 & control & r56 & r32 \\
\hline 8 & y66 & yy44 & yy42 & ww41 & ww20 & pp28 & b43 & $r 49$ & r32 \\
\hline 9 & y44 & yу38 & yy43 & ww41 & ww32 & pp34 & b46 & $r 48$ & $r 48$ \\
\hline 10 & y100 & yy42 & yy45 & ww72 & ww53 & pp52 & b53 & r38 & r52 \\
\hline 11 & $\mathrm{y} 54$ & yy28 & yy53 & ww46 & ww37 & pp41 & b34 & r51 & r29 \\
\hline 12 & y58 & yy50 & yy 45 & ww42 & ww50 & pp36 & b38 & r25 & r20 \\
\hline 13 & y53 & yy42 & yy28 & ww34 & ww26 & pp28 & b50 & control & r71 \\
\hline 14 & y55 & yy46 & control & control & ww36 & pp29 & b28 & bb24 & r37 \\
\hline 15 & y55 & yy24 & w54 & w32 & control & pp47 & b36 & bb20 & r34 \\
\hline 16 & $\mathrm{y} 63$ & control & w38 & w49 & p60 & pp25 & b57 & bb39 & r52 \\
\hline 17 & $y 77$ & y52 & w46 & w55 & $\mathrm{p} 42$ & pp48 & b42 & bb56 & r38 \\
\hline 18 & y34 & y50 & w40 & w56 & $\mathrm{p} 43$ & pp80 & b39 & bb33 & r83 \\
\hline 19 & $\mathrm{y} 51$ & y36 & w60 & w40 & p38 & pp62 & b47 & bb34 & missing \\
\hline 20 & y93 & y63 & w51 & w57 & p32 & pp40 & b35 & bb64 & r34 \\
\hline 21 & & y49 & w51 & w49 & p52 & pp60 & b45 & bb40 & $r 40$ \\
\hline 22 & & y48 & w41 & w54 & p30 & control & b56 & bb38 & r31 \\
\hline 23 & & $\mathrm{y} 48$ & w58 & w49 & $\mathrm{p} 40$ & $\mathrm{p} 42$ & b52 & bb62 & $r 48$ \\
\hline 24 & & & w25 & w37 & $\mathrm{p} 40$ & $\mathrm{p} 45$ & b46 & bb62 & r36 \\
\hline 25 & & & w69 & w57 & p28 & p50 & b40 & bb34 & r55 \\
\hline 26 & & & & w45 & p45 & p50 & b51 & bb45 & $r 40$ \\
\hline 27 & & & & w37 & p30 & $\mathrm{p} 48$ & b47 & bb38 & r54 \\
\hline 28 & & & & w46 & p57 & p54 & b80 & bb42 & $\mathrm{r} 41$ \\
\hline 29 & & & & & p38 & p53 & b52 & bb47 & r50 \\
\hline 30 & & & & & p73 & p44 & b37 & bb63 & r37 \\
\hline 31 & & & & & & p51 & b28 & bb34 & r66 \\
\hline 32 & & & NORTH & & & p75 & b80 & bb53 & r38 \\
\hline 33 & & WEST & + & EAST & & & control & bb63 & r34 \\
\hline 34 & & & SOUTH & & & & missing & bb53 & r83 \\
\hline 35 & & & & & & & bb10 & bb62 & r57 \\
\hline 36 & & & & & & & stunted & bb54 & r33 \\
\hline 37 & & & & & & & & bb88 & r56 \\
\hline 38 & & & & & & & & & r39 \\
\hline 39 & & & & & & & & & r38 \\
\hline
\end{tabular}


Figure 10. 2002 growing season.

10-12-02 Number of clusters harvested/vine.

\begin{tabular}{|c|c|c|c|c|c|c|c|c|c|}
\hline & 1 & $\underline{2}$ & $\underline{3}$ & $\underline{4}$ & $\underline{5}$ & $\underline{6}$ & $\underline{7}$ & $\underline{8}$ & $\underline{9}$ \\
\hline 1 & & & & & & & & r30 & r22 \\
\hline 2 & $\mathrm{y} 4$ & yy7 & yy7 & ww15 & ww12 & pp22 & pp24 & $r 18$ & r23 \\
\hline 3 & y7 & yy7 & yy7 & ww9 & ww17 & pp25 & pp19 & r31 & r30 \\
\hline 4 & y8 & yy9 & yy6 & ww15 & ww16 & $\mathrm{pp} 11$ & pp24 & r33 & r31 \\
\hline 5 & y8 & yy5 & yy8 & ww15 & ww14 & pp22 & pp23 & r37 & r27 \\
\hline 6 & y3 & yy8 & yy7 & ww15 & ww14 & pp26 & pp25 & r34 & r32 \\
\hline 7 & missing & missing & yy7 & ww15 & ww10 & pp25 & control & r30 & r31 \\
\hline 8 & y6 & yy8 & yy7 & ww14 & ww12 & pp23 & b29 & r36 & r37 \\
\hline 9 & $\mathrm{y} 11$ & yy4 & yy6 & ww13 & ww15 & pp23 & b30 & r23 & r23 \\
\hline 10 & $\mathrm{y} 6$ & yy9 & yy9 & ww15 & ww14 & pp30 & b27 & r32 & r32 \\
\hline 11 & y8 & yy7 & yy9 & ww14 & ww18 & pp20 & b30 & r37 & r37 \\
\hline 12 & y8 & yy7 & yy10 & ww13 & ww25 & pp28 & b31 & r34 & r20 \\
\hline 13 & y7 & yy8 & yy20 & ww11 & ww13 & pp23 & b29 & control & $\mathrm{r} 26$ \\
\hline 14 & y6 & yy7 & control & control & ww14 & $\mathrm{pp} 16$ & b19 & bb26 & r38 \\
\hline 15 & y8 & yy6 & w14 & w14 & control & pp16 & b29 & bb22 & $\mathrm{r} 15$ \\
\hline 16 & y7 & control & w13 & w13 & p17 & pp17 & b27 & bb30 & r38 \\
\hline 17 & y6 & y6 & w14 & w16 & p24 & pp19 & b24 & bb35 & r29 \\
\hline 18 & y9 & y10 & w16 & w13 & p17 & pp18 & b26 & bb21 & r35 \\
\hline 19 & $\mathrm{y} 8$ & y8 & w16 & w11 & p24 & pp18 & b29 & bb29 & missing \\
\hline 20 & y9 & y9 & w11 & w19 & p18 & pp18 & b27 & bb28 & r35 \\
\hline 21 & & y8 & w15 & w16 & p21 & pp22 & b21 & bb26 & r21 \\
\hline 22 & & y6 & w13 & w15 & p21 & control & b28 & bb33 & r34 \\
\hline 23 & & $y 7$ & w14 & w14 & p23 & $\mathrm{p} 16$ & b26 & bb27 & $\mathrm{r} 24$ \\
\hline 24 & & & w12 & w14 & p21 & p23 & b25 & bb20 & r25 \\
\hline 25 & & & w9 & w14 & p21 & p20 & b28 & bb18 & r36 \\
\hline 26 & & & & w15 & p17 & $\mathrm{p} 11$ & b26 & bb29 & r22 \\
\hline 27 & & & & w13 & p24 & p16 & b29 & bb28 & r35 \\
\hline 28 & & & & w14 & p22 & $\mathrm{p} 15$ & b24 & bb20 & $\mathrm{r} 28$ \\
\hline 29 & & & & & p16 & p19 & b25 & bb25 & $r 42$ \\
\hline 30 & & & & & p20 & p20 & b36 & bb32 & r32 \\
\hline 31 & & & & & & $\mathrm{p} 11$ & b22 & bb22 & r32 \\
\hline 32 & & & NORTH & & & $\mathrm{p} 22$ & b26 & bb20 & $\mathrm{r} 24$ \\
\hline 33 & & WEST & + & EAST & & & control & bb26 & r20 \\
\hline 34 & & & SOUTH & & & & missing & bb27 & $\mathrm{r} 40$ \\
\hline 35 & & & & & & & bb28 & bb25 & r38 \\
\hline 36 & & & & & & & stunted & bb26 & r21 \\
\hline 37 & & & & & & & & bb29 & r29 \\
\hline 38 & & & & & & & & & $\mathrm{r} 17$ \\
\hline 39 & & & & & & & & & r37 \\
\hline
\end{tabular}


Figure 11. 2002 growing season.

10-12-02 Ounces of harvested fruit/vine.

\begin{tabular}{|c|c|c|c|c|c|c|c|c|c|}
\hline & $\underline{1}$ & $\underline{2}$ & $\underline{3}$ & $\underline{4}$ & $\underline{\mathbf{5}}$ & $\underline{6}$ & $\underline{7}$ & $\underline{8}$ & $\underline{9}$ \\
\hline 1 & & & & & & & & r83 & r60 \\
\hline 2 & y14 & yy19 & yy17 & ww41 & ww29 & pp56 & pp82 & $\mathrm{r} 27$ & r56 \\
\hline 3 & y20 & yy23 & yy25 & ww17 & ww41 & pp66 & pp52 & r103 & r75 \\
\hline 4 & y30 & yy28 & yy20 & ww36 & ww48 & pp19 & pp76 & r76 & r70 \\
\hline 5 & $\mathrm{y} 16$ & yy11 & yy24 & ww30 & ww32 & p58 & pp68 & r125 & r66 \\
\hline 6 & y5 & yy27 & yy17 & ww39 & ww38 & pp86 & pp57 & r92 & r99 \\
\hline 7 & missing & missing & yy26 & ww33 & ww28 & pp77 & control & r70 & r84 \\
\hline 8 & $\mathrm{y} 12$ & yy24 & yy21 & ww32 & ww32 & pp62 & b103 & r88 & r120 \\
\hline 9 & y32 & yy14 & yy15 & ww34 & ww40 & pp70 & b87 & r69 & r72 \\
\hline 10 & $\mathrm{y} 12$ & yy28 & yy25 & ww42 & ww36 & pp89 & b86 & r78 & r106 \\
\hline 11 & y27 & yy16 & yy26 & ww32 & ww55 & pp45 & b104 & r129 & r120 \\
\hline 12 & y33 & yy22 & yy32 & ww31 & ww44 & pp64 & b100 & r89 & r44 \\
\hline 13 & y19 & yy29 & yy60 & ww16 & ww35 & pp61 & b88 & control & r53 \\
\hline 14 & $\mathrm{y} 20$ & yy26 & control & control & ww41 & $\mathrm{pp} 41$ & b73 & bb52 & $r 96$ \\
\hline 15 & y27 & yy17 & w23 & w28 & control & pp46 & b93 & bb56 & r38 \\
\hline 16 & y18 & control & w34 & w36 & p52 & $\mathrm{pp} 43$ & b89 & bb80 & $r 113$ \\
\hline 17 & $\mathrm{y} 17$ & y31 & w36 & w43 & p66 & pp47 & b82 & bb82 & r87 \\
\hline 18 & y20 & $\mathrm{y} 27$ & w35 & w34 & p44 & pp39 & b69 & bb54 & r96 \\
\hline 19 & y22 & y25 & w47 & w22 & p68 & pp35 & b100 & bb61 & missing \\
\hline 20 & y33 & y34 & w28 & w47 & p56 & pp51 & b96 & bb69 & r84 \\
\hline 21 & & y28 & w37 & w37 & p47 & pp51 & b54 & bb59 & r56 \\
\hline 22 & & y19 & w32 & w34 & p52 & control & b67 & bb75 & $r 77$ \\
\hline 23 & & $\mathrm{y} 22$ & w36 & w29 & p64 & p43 & b72 & bb59 & $r 64$ \\
\hline 24 & & & w25 & w35 & p40 & p48 & b74 & bb45 & r40 \\
\hline 25 & & & w31 & w42 & $\mathrm{p} 40$ & p49 & b62 & bb36 & r81 \\
\hline 26 & & & & w45 & p45 & p29 & b88 & bb77 & r55 \\
\hline 27 & & & & w41 & p47 & p42 & b66 & $\mathrm{bb} 60$ & r69 \\
\hline 28 & & & & w34 & p53 & $\mathrm{p} 43$ & b52 & bb43 & r66 \\
\hline 29 & & & & & p46 & p49 & b63 & bb65 & r93 \\
\hline 30 & & & & & p64 & p36 & b92 & bb82 & r66 \\
\hline 31 & & & & & & p24 & b40 & bb37 & $\mathrm{r} 50$ \\
\hline 32 & & & NORTH & & & p76 & b90 & bb37 & $\mathrm{r} 41$ \\
\hline 33 & & WEST & + & EAST & & & control & bb64 & r51 \\
\hline 34 & & & SOUTH & & & & missing & bb56 & $r 110$ \\
\hline 35 & & & & & & & bb49 & bb70 & r91 \\
\hline 36 & & & & & & & stunted & bb58 & r41 \\
\hline 37 & & & & & & & & bb63 & $r 74$ \\
\hline 38 & & & & & & & & & r27 \\
\hline 39 & & & & & & & & & $\mathrm{r} 112$ \\
\hline
\end{tabular}


Figure 12. 2003 growing season.

2-5-04 Trial site map containing number of canes pruned/vine.

\begin{tabular}{|c|c|c|c|c|c|c|c|c|c|}
\hline & 1 & $\underline{2}$ & $\underline{3}$ & $\underline{4}$ & $\underline{5}$ & $\underline{6}$ & $\underline{7}$ & $\underline{8}$ & $\underline{9}$ \\
\hline 1 & & & & & & & & r18 & r21 \\
\hline 2 & $\mathrm{y} 13$ & yy20 & yy26 & ww18 & ww20 & pp24 & pp23 & r9 & $\mathrm{r} 19$ \\
\hline 3 & $y 14$ & yy23 & yy17 & ww19 & ww30 & pp20 & pp9 & r21 & $r 19$ \\
\hline 4 & y19 & yy17 & yy19 & ww19 & ww19 & pp14 & pp19 & $\mathrm{r} 17$ & $r 18$ \\
\hline 5 & y20 & yy17 & yy30 & ww19 & ww15 & pp18 & pp22 & $\mathrm{r} 20$ & r20 \\
\hline 6 & y19 & yy23 & yy18 & ww19 & ww22 & pp14 & pp20 & r19 & r21 \\
\hline 7 & missing & missing & yy17 & ww20 & ww26 & pp18 & control & $\mathrm{r} 19$ & r22 \\
\hline 8 & $\mathrm{y} 21$ & yy25 & yy19 & ww14 & ww17 & pp17 & b21 & $\mathrm{r} 18$ & $\mathrm{r} 17$ \\
\hline 9 & $y 23$ & yy20 & yy19 & ww18 & ww14 & pp15 & b21 & r20 & r20 \\
\hline 10 & $\mathrm{y} 22$ & yy27 & yy17 & ww20 & ww19 & pp29 & b28 & $\mathrm{r} 25$ & $\mathrm{r} 19$ \\
\hline 11 & y24 & yy17 & yy17 & ww15 & ww16 & pp15 & b13 & $\mathrm{r} 15$ & $\mathrm{r} 21$ \\
\hline 12 & $\mathrm{y} 16$ & yy26 & yy20 & ww13 & ww22 & pp19 & b18 & r24 & r16 \\
\hline 13 & $\mathrm{y} 16$ & yy15 & yy16 & ww17 & ww15 & pp23 & b18 & control & r22 \\
\hline 14 & $\mathrm{y} 13$ & yy21 & control & control & ww14 & pp16 & b8 & bb18 & r24 \\
\hline 15 & $\mathrm{y} 17$ & yy19 & w19 & w22 & control & pp16 & b17 & bb18 & $\mathrm{r} 19$ \\
\hline 16 & $\mathrm{y} 21$ & control & w21 & w19 & p26 & pp20 & b23 & bb20 & r24 \\
\hline 17 & y22 & y13 & w30 & w21 & p21 & pp20 & b22 & bb20 & r20 \\
\hline 18 & y15 & y20 & w17 & w20 & p19 & pp25 & b20 & bb18 & r26 \\
\hline 19 & $\mathrm{y} 18$ & $\mathrm{y} 14$ & w31 & w20 & p20 & pp18 & b15 & $b b 23$ & missing \\
\hline 20 & y22 & $\mathrm{y} 24$ & w15 & w24 & p25 & pp17 & b21 & bb19 & r20 \\
\hline 21 & & y18 & w23 & w20 & p28 & pp20 & b20 & bb23 & r21 \\
\hline 22 & & y23 & w24 & w21 & p18 & control & b20 & bb18 & r21 \\
\hline 23 & & y19 & w19 & w23 & p24 & p17 & b21 & $\mathrm{bb} 26$ & $\mathrm{r} 19$ \\
\hline 24 & & & w17 & w16 & p27 & p19 & b22 & bb14 & $\mathrm{r} 19$ \\
\hline 25 & & & w30 & w20 & p15 & p30 & b17 & bb18 & r21 \\
\hline 26 & & & & w20 & p19 & p21 & b20 & bb24 & r28 \\
\hline 27 & & & & w17 & p21 & p19 & b17 & bb27 & r20 \\
\hline 28 & & & & w16 & p23 & p19 & b27 & bb17 & $\mathrm{r} 19$ \\
\hline 29 & & & & & p28 & p22 & b15 & bb25 & $\mathrm{r} 16$ \\
\hline 30 & & & & & p31 & p21 & b16 & bb20 & r26 \\
\hline 31 & & & & & & p12 & b13 & bb25 & r29 \\
\hline 32 & & & NORTH & & & p21 & b25 & $\mathrm{bb} 26$ & r20 \\
\hline 33 & & WEST & + & EAST & & & control & bb21 & $\mathrm{r} 15$ \\
\hline 34 & & & SOUTH & & & & missing & bb19 & r29 \\
\hline 35 & & & & & & & bb21 & bb19 & r24 \\
\hline 36 & & & & & & & stunted & bb21 & r25 \\
\hline 37 & & & & & & & & bb28 & r21 \\
\hline 38 & & & & & & & & & r23 \\
\hline 39 & & & & & & & & & r15 \\
\hline
\end{tabular}


Figure 13. 2003 growing season.

2-5-04 Trial site map containing ounces of prunings/vine.

\begin{tabular}{|c|c|c|c|c|c|c|c|c|c|}
\hline & 1 & $\underline{2}$ & $\underline{3}$ & $\underline{4}$ & $\underline{5}$ & $\underline{6}$ & $\underline{7}$ & $\underline{8}$ & $\underline{9}$ \\
\hline 1 & & & & & & & & r37 & r52 \\
\hline 2 & y49 & yy56 & yy40 & ww50 & ww45 & pp50 & pp54 & r12 & r48 \\
\hline 3 & y22 & yy40 & yy46 & ww52 & ww52 & pp34 & pp28 & r65 & $r 42$ \\
\hline 4 & y65 & yy34 & yy42 & ww26 & ww40 & pp16 & pp46 & $r 49$ & r38 \\
\hline 5 & y60 & yy61 & yy55 & ww44 & ww26 & pp36 & pp29 & $r 46$ & $r 46$ \\
\hline 6 & y66 & yy47 & yy32 & ww42 & ww54 & pp46 & pp82 & r33 & r52 \\
\hline 7 & missing & missing & yy52 & ww27 & ww52 & pp31 & control & r57 & $r 48$ \\
\hline 8 & y49 & yy58 & yy55 & ww38 & ww36 & pp23 & b46 & r50 & r49 \\
\hline 9 & y42 & yy44 & yy33 & ww36 & ww38 & pp25 & b54 & r45 & r47 \\
\hline 10 & y92 & yy60 & yy46 & ww66 & ww56 & pp60 & b69 & $\mathrm{r} 42$ & $r 43$ \\
\hline 11 & y65 & yy42 & yy62 & ww26 & ww38 & $\mathrm{pp} 42$ & b26 & $\mathrm{r} 45$ & r52 \\
\hline 12 & y45 & yy52 & yy47 & ww30 & ww58 & pp36 & b39 & r28 & r45 \\
\hline 13 & $\mathrm{y} 44$ & yy43 & yy31 & ww43 & ww20 & pp33 & b56 & control & r44 \\
\hline 14 & $y 42$ & yy46 & control & control & ww34 & pp28 & b22 & bb27 & r58 \\
\hline 15 & y58 & yy33 & w54 & w26 & control & $\mathrm{pp} 43$ & b49 & bb29 & r54 \\
\hline 16 & y66 & control & w53 & w47 & p63 & pp29 & b58 & bb42 & $r 45$ \\
\hline 17 & y76 & y52 & w50 & w43 & p50 & pp42 & b52 & bb46 & $r 46$ \\
\hline 18 & y20 & y40 & w46 & w54 & p45 & pp61 & b44 & bb33 & r48 \\
\hline 19 & $\mathrm{y} 40$ & $\mathrm{y} 30$ & w57 & w42 & p51 & pp44 & b52 & bb39 & missing \\
\hline 20 & y76 & y54 & w46 & w54 & p39 & pp24 & b39 & bb77 & $\mathrm{r} 47$ \\
\hline 21 & & y46 & w56 & w43 & p69 & pp36 & b54 & bb48 & r50 \\
\hline 22 & & y34 & w45 & w50 & p38 & control & b60 & bb28 & r39 \\
\hline 23 & & $\mathrm{y} 40$ & w54 & w42 & p55 & p36 & b59 & bb82 & $r 45$ \\
\hline 24 & & & w24 & w33 & p47 & p33 & b50 & bb53 & r39 \\
\hline 25 & & & w74 & w47 & p31 & p38 & b40 & bb36 & r54 \\
\hline 26 & & & & w46 & p49 & p43 & b56 & bb54 & r39 \\
\hline 27 & & & & w33 & p22 & $\mathrm{p} 40$ & b34 & bb39 & r56 \\
\hline 28 & & & & w38 & p59 & p45 & b86 & $\mathrm{bb} 40$ & r54 \\
\hline 29 & & & & & p55 & p34 & b54 & bb50 & r50 \\
\hline 30 & & & & & p65 & p37 & b36 & bb59 & $r 46$ \\
\hline 31 & & & & & & p49 & b30 & $\mathrm{bb} 40$ & r66 \\
\hline 32 & & & NORTH & & & p58 & b77 & $\mathrm{bb} 66$ & r55 \\
\hline 33 & & WEST & + & EAST & & & control & bb52 & $r 48$ \\
\hline 34 & & & SOUTH & & & & missing & bb52 & r70 \\
\hline 35 & & & & & & & bb49 & bb60 & r53 \\
\hline 36 & & & & & & & stunted & bb40 & $r 48$ \\
\hline 37 & & & & & & & & bb70 & r42 \\
\hline 38 & & & & & & & & & r56 \\
\hline 39 & & & & & & & & & r34 \\
\hline
\end{tabular}


Figure 14. 2003 growing season.

9-29-03 Number of clusters harvested/vine.

\begin{tabular}{|c|c|c|c|c|c|c|c|c|c|}
\hline \multicolumn{2}{|r|}{1} & \multirow[t]{2}{*}{$\underline{2}$} & \multicolumn{2}{|r|}{$\underline{4}$} & \multirow[t]{2}{*}{$\underline{5}$} & \multirow[t]{2}{*}{$\underline{6}$} & $\underline{7}$ & $\underline{8}$ & \multirow{2}{*}{$r 30^{\underline{9}}$} \\
\hline 1 & & & & & & & & r34 & \\
\hline 2 & $y 7$ & YY9 & yy9 & ww15 & ww18 & pp22 & pp25 & $r 16$ & r31 \\
\hline 3 & Y6 & YY10 & yy11 & ww13 & ww18 & pp24 & pp14 & r36 & $\mathrm{r} 42$ \\
\hline 4 & Y8 & yy9 & yy11 & ww14 & ww17 & pp22 & pp24 & r28 & r27 \\
\hline 5 & Y8 & YY8 & yy15 & ww18 & ww16 & pp24 & pp22 & r32 & r34 \\
\hline 6 & Y8 & yy8 & yy8 & ww22 & ww15 & pp23 & pp25 & r29 & r26 \\
\hline 7 & missing & missing & yy8 & ww17 & ww18 & pp22 & control & r34 & $\mathrm{r} 41$ \\
\hline 8 & Y8 & yy8 & yy11 & ww20 & ww18 & pp30 & b30 & r34 & r23 \\
\hline 9 & Y9 & yy7 & yy7 & ww15 & ww20 & pp16 & b27 & r36 & r32 \\
\hline 10 & Y8 & yy9 & yy9 & ww18 & ww19 & pp31 & b32 & r36 & r35 \\
\hline 11 & Y8 & yy 10 & yy9 & ww20 & ww15 & pp28 & b22 & r27 & $r 43$ \\
\hline 12 & Y8 & yy8 & yy12 & ww15 & ww19 & pp18 & b26 & r44 & r38 \\
\hline 13 & Y8 & yy11 & yy7 & ww19 & ww18 & pp24 & b29 & control & r28 \\
\hline 14 & Y5 & yy6 & control & control & ww20 & pp20 & b13 & bb30 & r37 \\
\hline 15 & $\mathrm{Y} 10$ & yy10 & w8 & w17 & control & pp18 & b18 & bb24 & $\mathrm{r} 28$ \\
\hline 16 & Y9 & control & w16 & w16 & p32 & pp20 & b30 & bb38 & r37 \\
\hline 17 & Y8 & $y 11$ & w16 & w14 & p23 & pp23 & b25 & bb28 & r24 \\
\hline 18 & $\mathrm{Y} 11$ & $\mathrm{y} 12$ & w12 & w15 & p18 & pp27 & b25 & bb41 & r37 \\
\hline 19 & Y6 & y5 & w22 & w9 & p22 & pp23 & b33 & bb35 & missing \\
\hline 20 & $Y 7$ & y8 & w13 & w16 & p21 & pp20 & b26 & bb26 & $r 43$ \\
\hline 21 & & $\mathrm{y} 10$ & w16 & w12 & p22 & pp27 & b26 & bb34 & r30 \\
\hline 22 & & y9 & w18 & w16 & p22 & control & b27 & bb22 & r36 \\
\hline 23 & & y5 & w18 & w16 & p21 & p36 & b32 & bb34 & r32 \\
\hline 24 & & & w11 & w13 & p29 & p33 & b30 & bb36 & r32 \\
\hline 25 & & & w14 & w16 & p16 & $\mathrm{p} 40$ & b24 & bb24 & r28 \\
\hline 26 & & & & w14 & $\mathrm{p} 20$ & p22 & b37 & bb25 & r36 \\
\hline 27 & & & & w12 & p28 & p24 & b31 & bb40 & $r 45$ \\
\hline 28 & & & & w15 & p23 & p32 & b34 & bb24 & r28 \\
\hline 29 & & & & & p18 & p28 & b21 & bb32 & r28 \\
\hline 30 & & & & & p22 & p28 & b34 & bb32 & r26 \\
\hline 31 & & & & & & p15 & b19 & $\mathrm{bb} 40$ & r42 \\
\hline 32 & & & NORTH & & & p20 & b35 & bb31 & r26 \\
\hline 33 & & WEST & + & EAST & & & control & bb28 & r25 \\
\hline 34 & & & SOUTH & & & & missing & bb23 & r22 \\
\hline 35 & & & & & & & bb34 & bb22 & r37 \\
\hline 36 & & & & & & & stunted & bb26 & r34 \\
\hline 37 & & & & & & & & bb34 & r27 \\
\hline 38 & & & & & & & & & r27 \\
\hline 39 & & & & & & & & & $\mathrm{r} 30$ \\
\hline
\end{tabular}


Figure 15. 2003 growing season.

9-29-03 Ounces of fruit harvested/vine.

\begin{tabular}{|c|c|c|c|c|c|c|c|c|c|}
\hline & 1 & $\underline{2}$ & $\underline{3}$ & $\underline{4}$ & $\underline{\mathbf{5}}$ & $\underline{6}$ & $\underline{7}$ & $\underline{8}$ & $\underline{9}$ \\
\hline 1 & & & & & & & & r51 & r49 \\
\hline 2 & $\mathrm{y} 11$ & yy16 & yy24 & ww44 & ww39 & pp46 & pp49 & r25 & r60 \\
\hline 3 & $\mathrm{y} 14$ & yy24 & yy24 & ww18 & ww54 & pp43 & pp24 & r72 & $r 76$ \\
\hline 4 & y20 & yy20 & yy28 & ww29 & ww42 & pp52 & pp50 & r41 & r46 \\
\hline 5 & y21 & yy16 & yy25 & ww35 & ww26 & pp66 & pp46 & r76 & r65 \\
\hline 6 & y10 & yy19 & yy20 & ww44 & ww31 & pp51 & pp46 & r60 & r56 \\
\hline 7 & missing & missing & yy20 & ww37 & ww50 & pp52 & control & r86 & r97 \\
\hline 8 & $\mathrm{y} 14$ & yy17 & yy22 & ww41 & ww35 & pp73 & b50 & r62 & r66 \\
\hline 9 & y20 & yy28 & yy13 & ww31 & ww51 & pp30 & b51 & r79 & r74 \\
\hline 10 & $\mathrm{y} 13$ & yy24 & yy25 & ww49 & ww39 & pp73 & b46 & r86 & r64 \\
\hline 11 & $\mathrm{y} 17$ & yy16 & yy23 & ww50 & ww24 & pp65 & b36 & r75 & r103 \\
\hline 12 & y19 & yy26 & yy33 & ww35 & ww46 & pp38 & b66 & r118 & r108 \\
\hline 13 & $\mathrm{y} 18$ & yy15 & yy18 & ww30 & ww35 & pp54 & b60 & control & r53 \\
\hline 14 & y14 & yy18 & control & control & ww57 & pp38 & b38 & bb70 & r55 \\
\hline 15 & $\mathrm{y} 12$ & yy13 & w13 & w53 & control & pp34 & b42 & bb53 & $\mathrm{r} 41$ \\
\hline 16 & $\mathrm{y} 13$ & control & w30 & w41 & p88 & pp45 & b85 & bb94 & r84 \\
\hline 17 & y13 & y24 & w52 & w40 & p60 & pp50 & b59 & bb63 & r28 \\
\hline 18 & y19 & y23 & w26 & w34 & p36 & pp56 & b70 & bb77 & r56 \\
\hline 19 & $\mathrm{y} 11$ & $\mathrm{y} 11$ & w64 & w11 & p56 & pp46 & b83 & bb76 & missing \\
\hline 20 & $\mathrm{y} 16$ & y22 & w23 & w36 & p68 & pp49 & b71 & bb49 & r94 \\
\hline 21 & & $\mathrm{y} 22$ & w33 & w33 & p61 & pp56 & b42 & bb66 & $r 70$ \\
\hline 22 & & y18 & w39 & w35 & p50 & control & b58 & bb40 & r56 \\
\hline 23 & & $\mathrm{y} 10$ & w36 & w34 & p45 & p73 & b54 & bb57 & $r 73$ \\
\hline 24 & & & w16 & w34 & p64 & p68 & b69 & bb55 & r68 \\
\hline 25 & & & w52 & w38 & p28 & p92 & b27 & bb46 & r56 \\
\hline 26 & & & & w30 & p63 & p60 & b73 & bb80 & $r 100$ \\
\hline 27 & & & & w23 & p65 & p54 & b63 & bb99 & r104 \\
\hline 28 & & & & w29 & p76 & p72 & b64 & bb43 & r51 \\
\hline 29 & & & & & p58 & p66 & b30 & bb60 & $\mathrm{r} 44$ \\
\hline 30 & & & & & p65 & p46 & b42 & bb81 & r34 \\
\hline 31 & & & & & & p33 & b24 & bb79 & $r 75$ \\
\hline 32 & & & NORTH & & & p64 & b84 & bb57 & r45 \\
\hline 33 & & WEST & + & EAST & & & control & bb55 & $\mathrm{r} 40$ \\
\hline 34 & & & SOUTH & & & & missing & bb37 & r30 \\
\hline 35 & & & & & & & bb62 & bb44 & r57 \\
\hline 36 & & & & & & & stunted & bb44 & r65 \\
\hline 37 & & & & & & & & bb62 & $r 40$ \\
\hline 38 & & & & & & & & & $r 46$ \\
\hline 39 & & & & & & & & & $r 48$ \\
\hline
\end{tabular}


Figure 16, Interaction Plot for TA from 2002 and 2003 growing seasons based on early and late fruit drop.

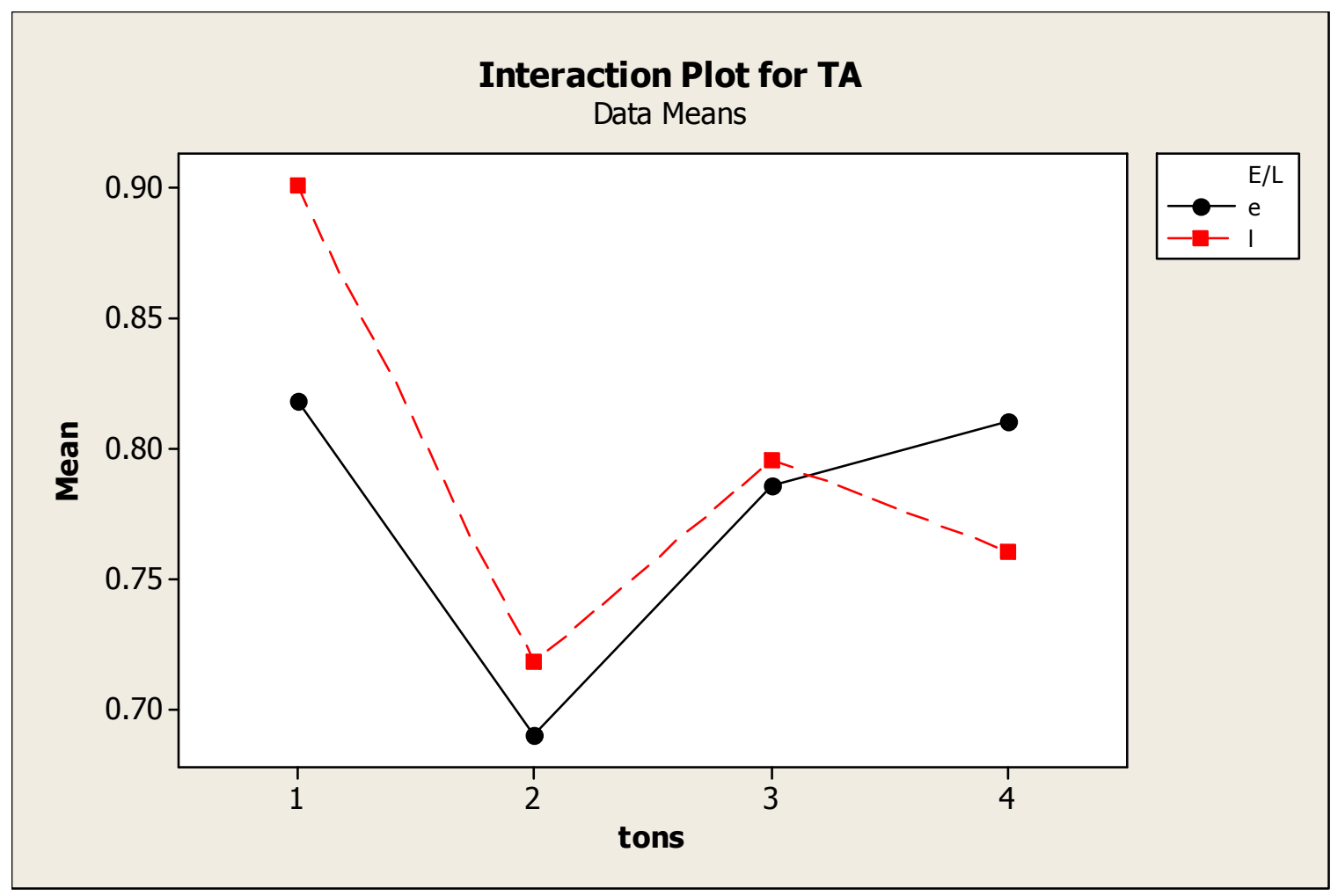


Figure 17, Interaction Plot for $\mathrm{pH}$ from 2002 and 2003 growing season based on early and late fruit drop.

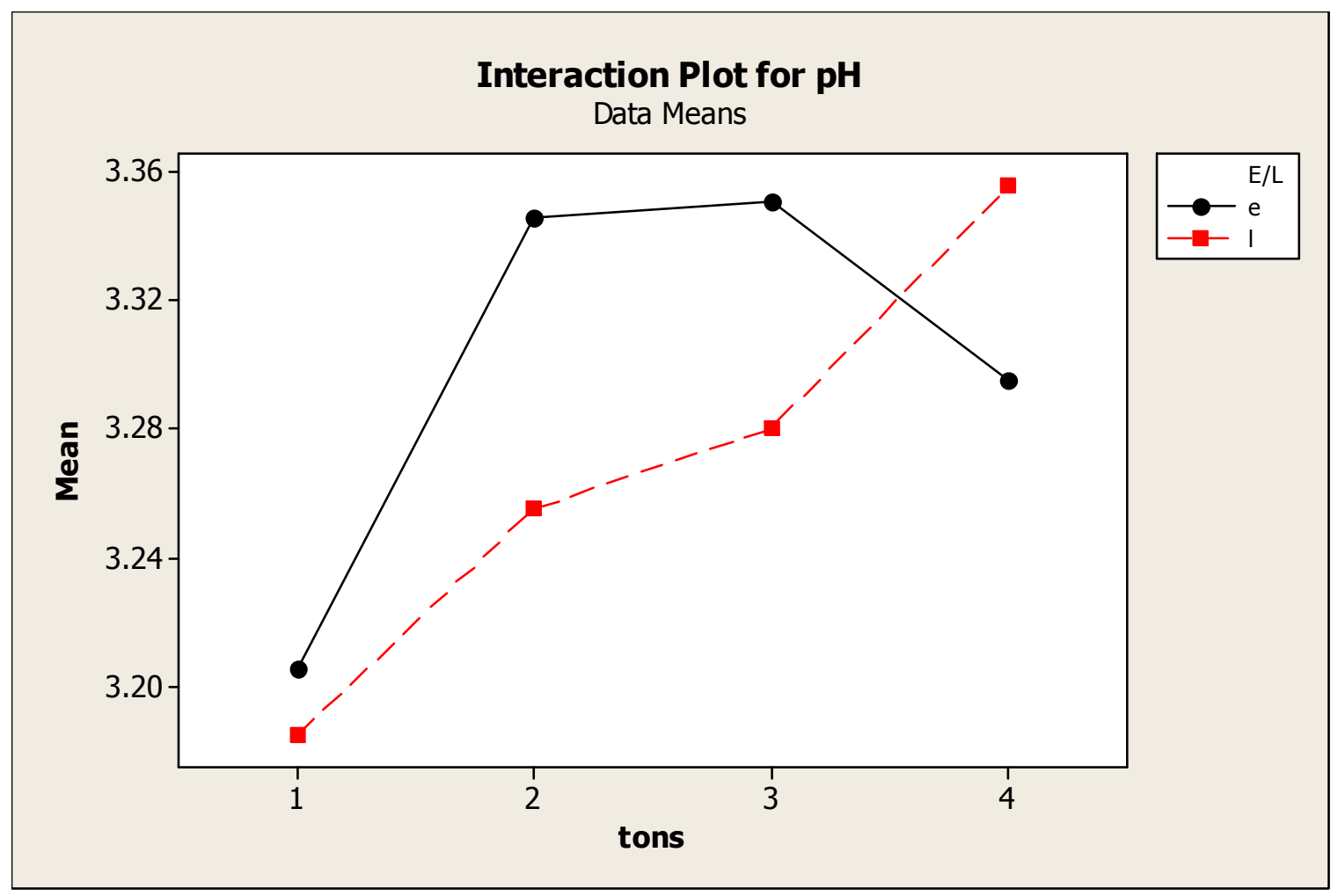


Figure 18, Interaction Plot for Brix (Total Soluble Solids) from 2002 and 2003 growing seasons based on early and late fruit drop.

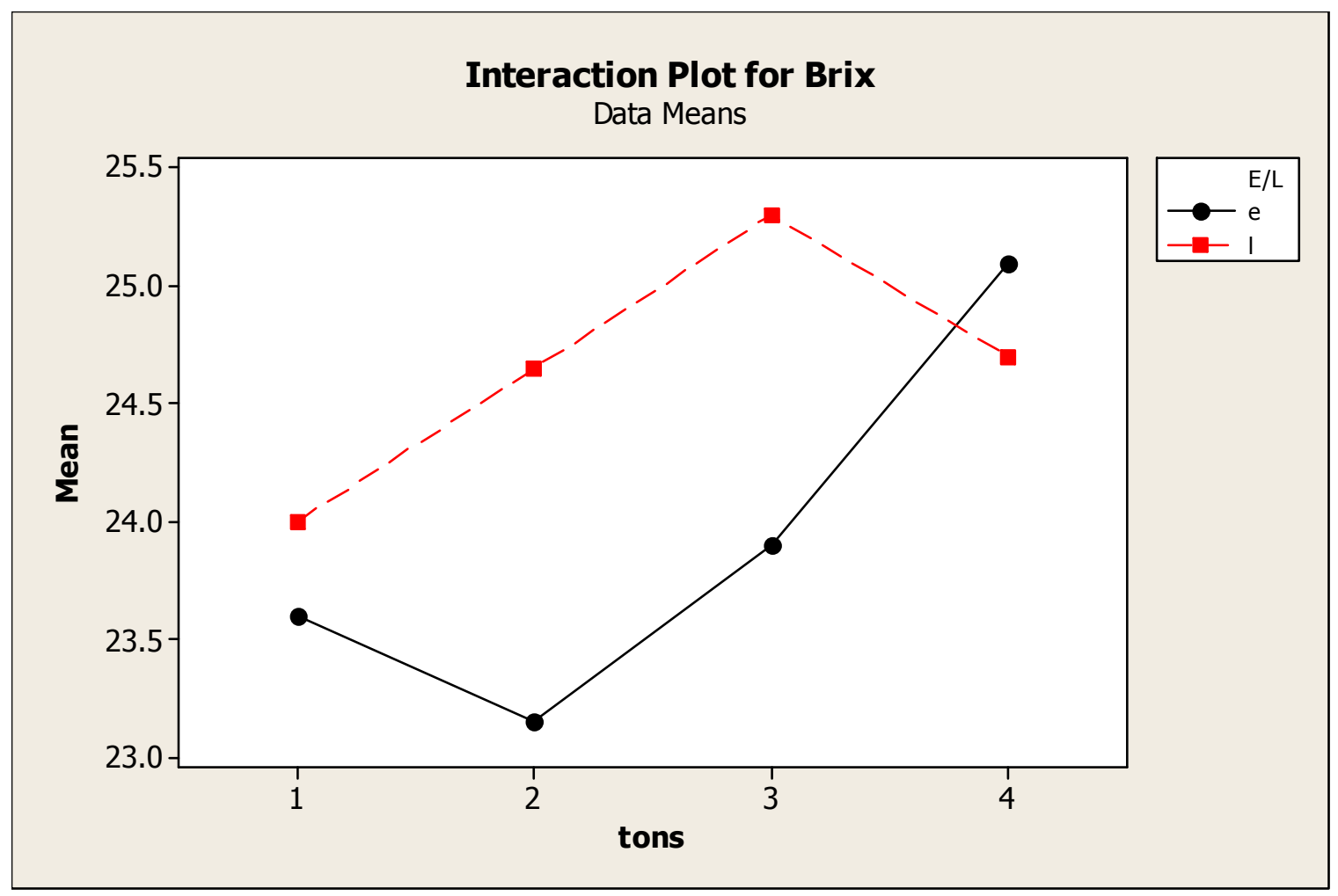


Figure 19, Interaction Plot for Berry Size from the 2002 growing season.

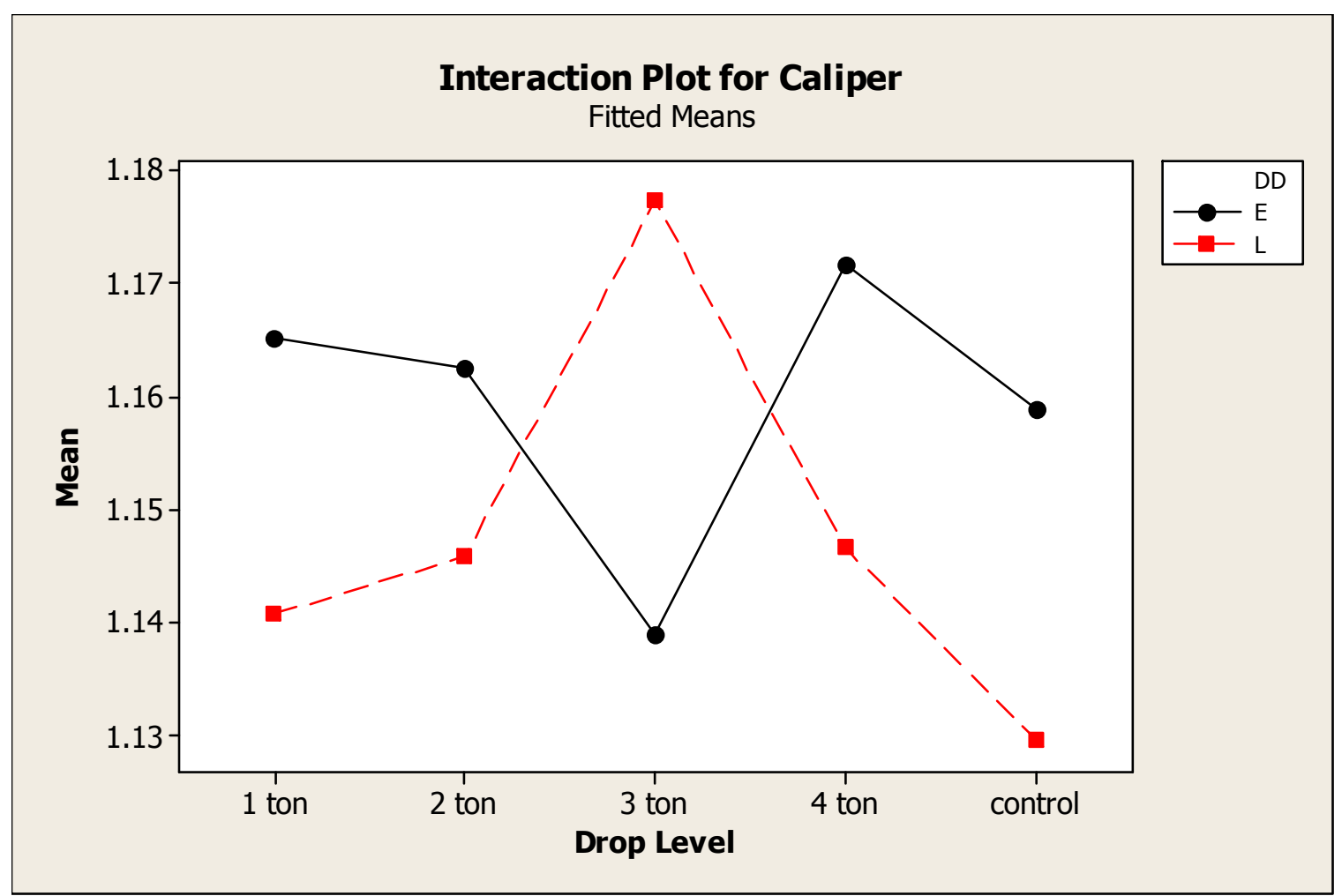


Figure 20, Interaction Plot for Berry Size from the 2003 growing season.

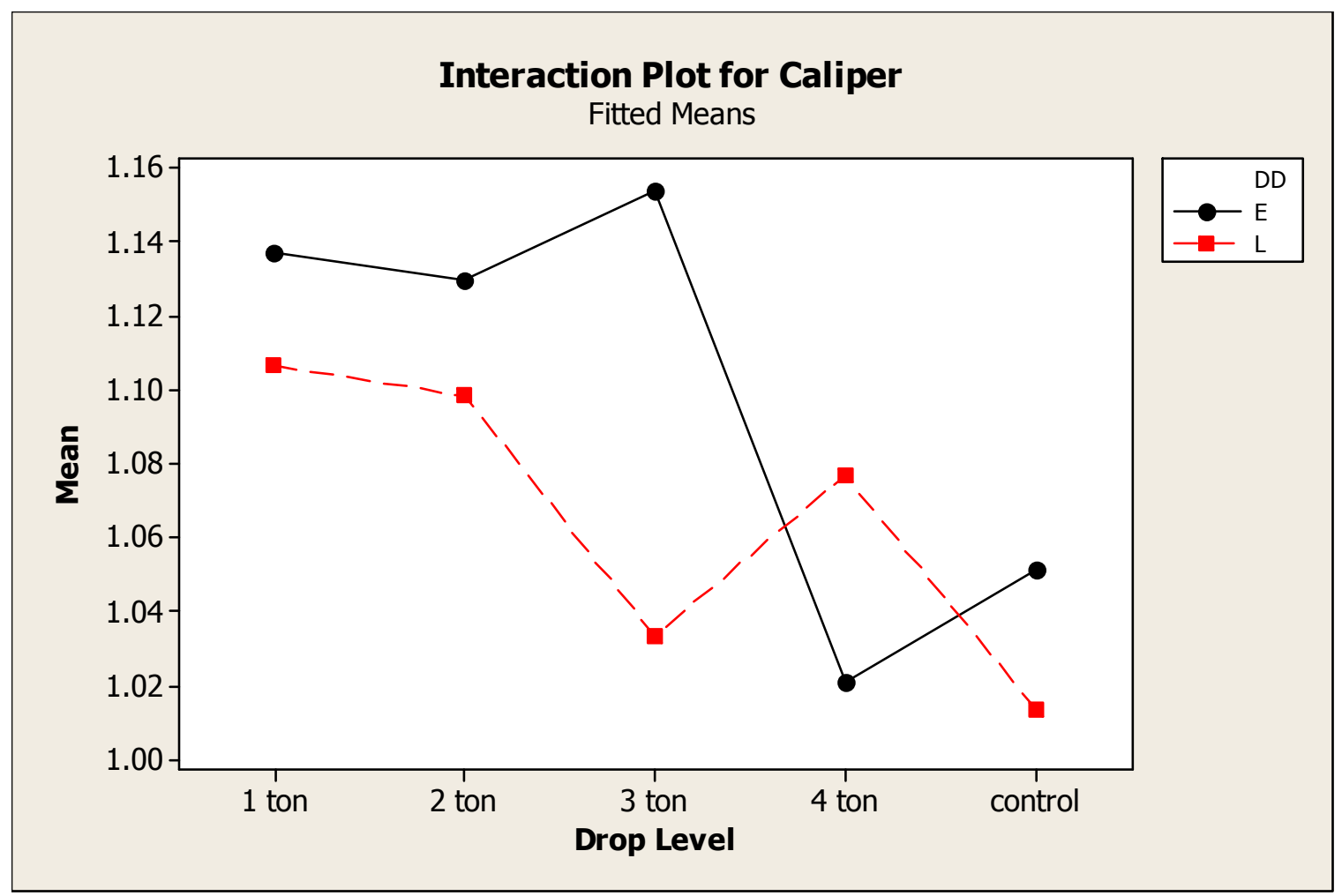


Figure 21, Interaction Plot for Average ounces/cluster/vine for the 2002 growing season based on early and late fruit drop.

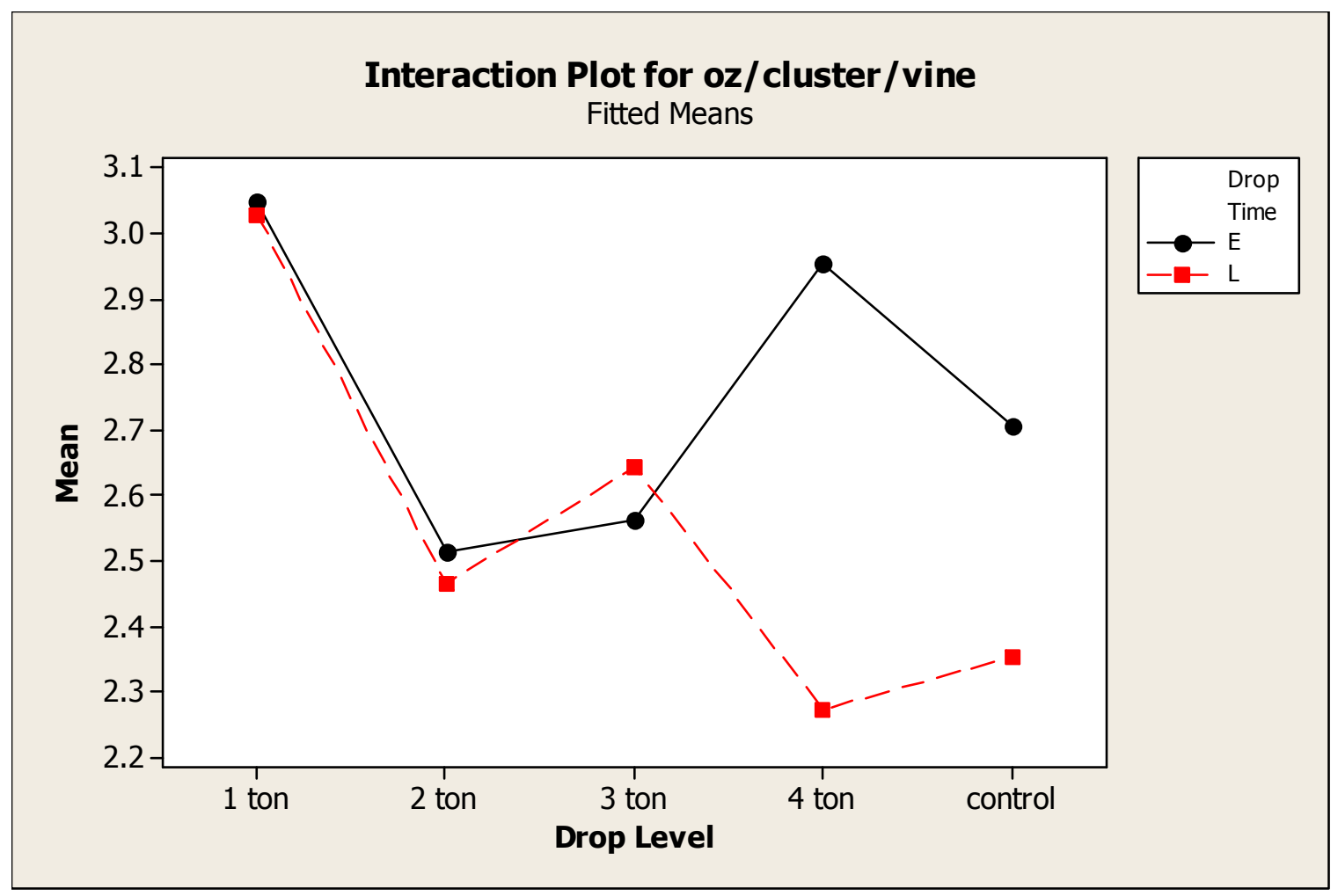


Figure 22, Interaction Plot for Average ounces/cluster/vine for the 2003 growing season based on early and late fruit drop.

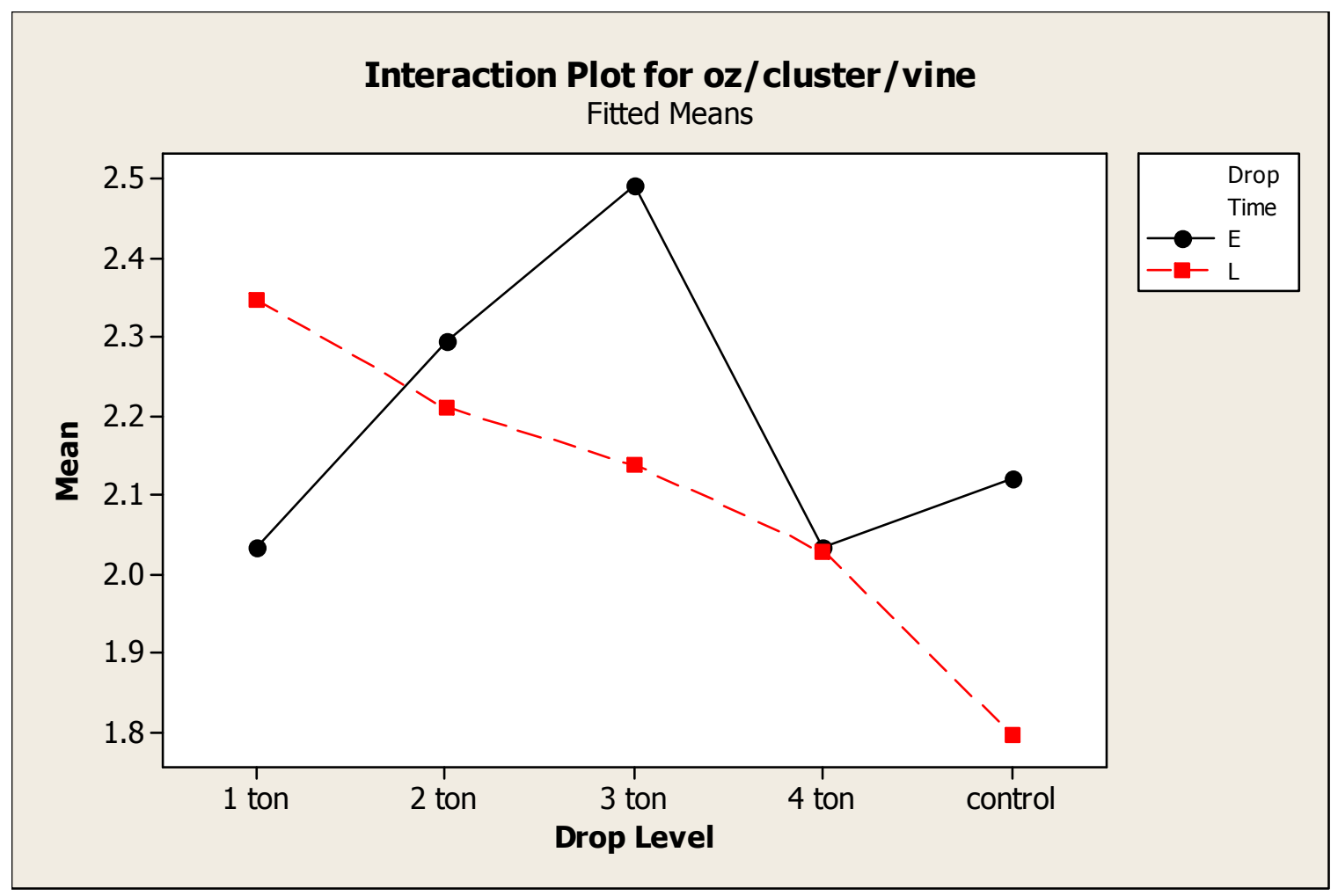


Figure 23, Interaction Plot for the Average ounces/shoot/vine for 2002 growing season based on early and late fruit drop.

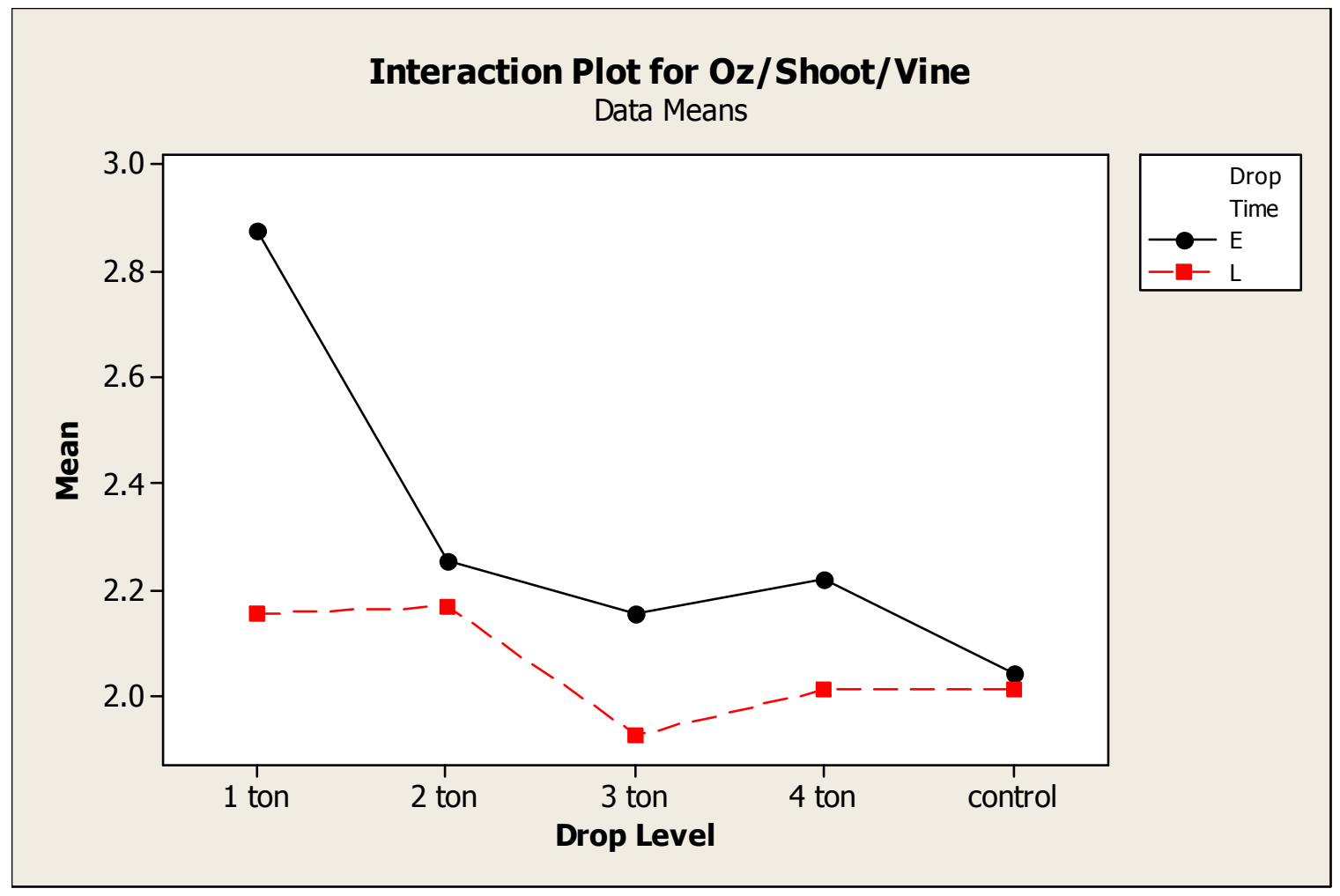


Figure 24, Interaction Plot for Average ounces/shoot/vine for 2003 growing season based on early and late fruit drop.

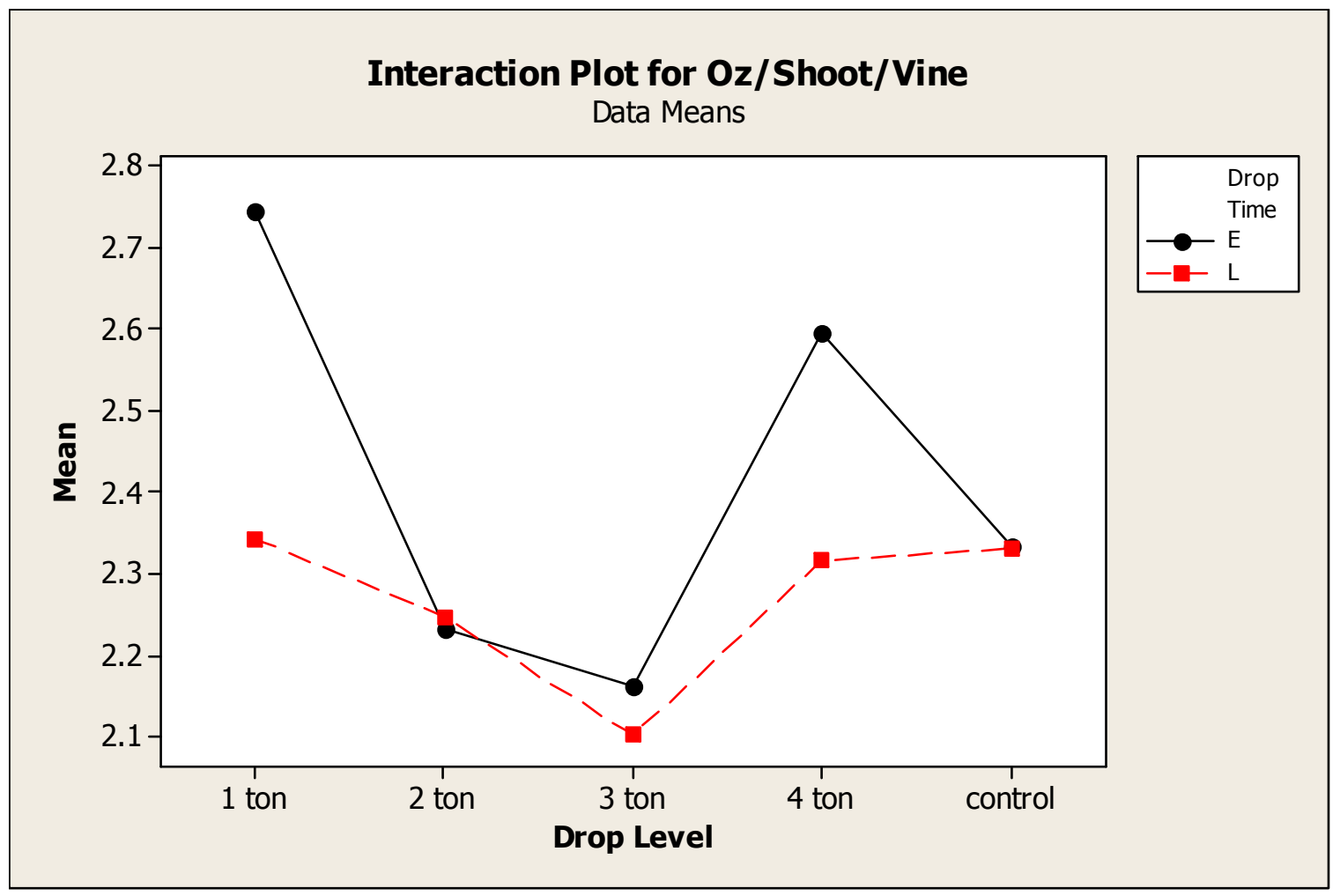


Figure 25, Scatterplot of gallic acid versus ttt based on 2002 and 2003 growing seasons

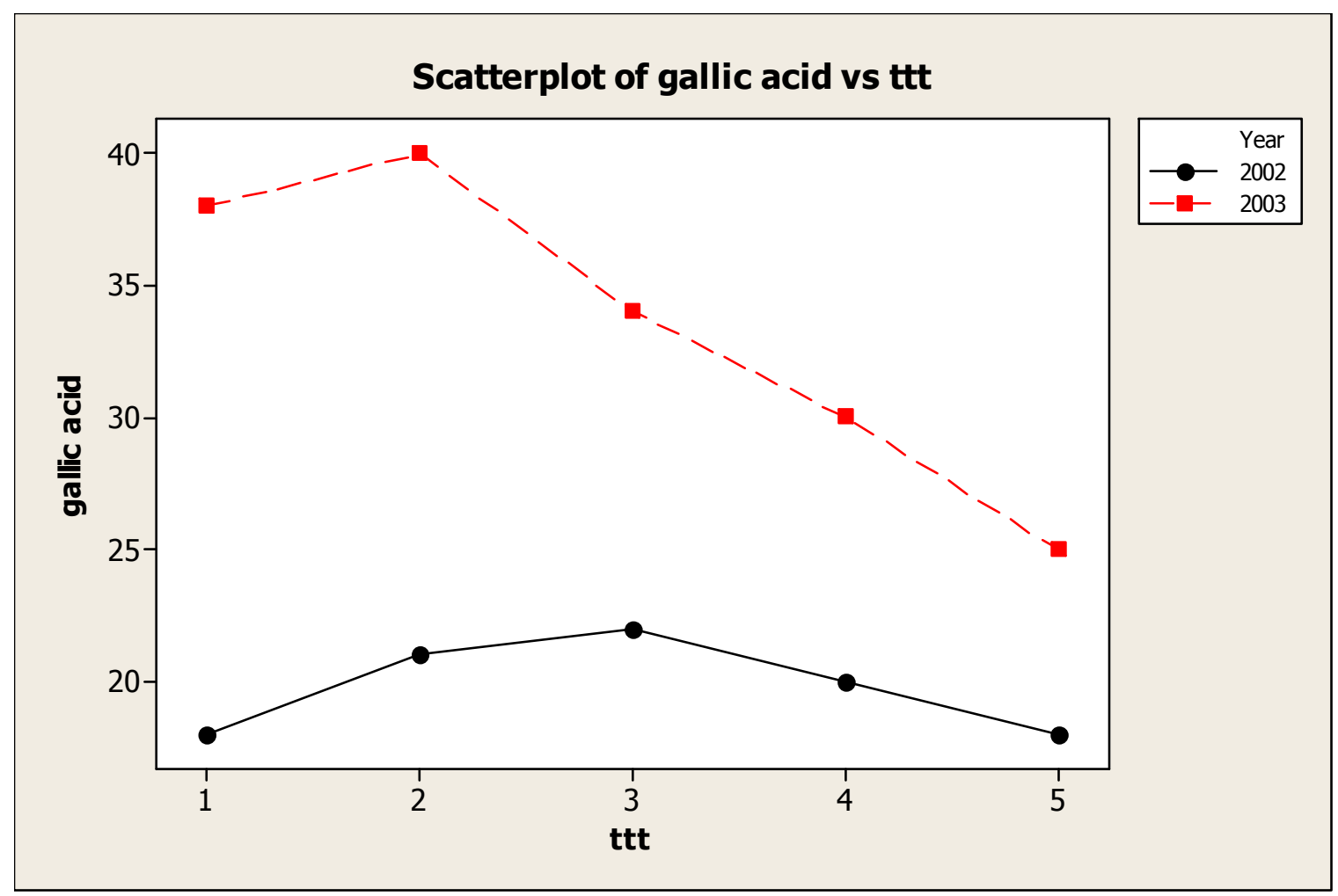


Figure 26, Scatterplot of catechin versus ttt based on 2002 and 2003 growing seasons.

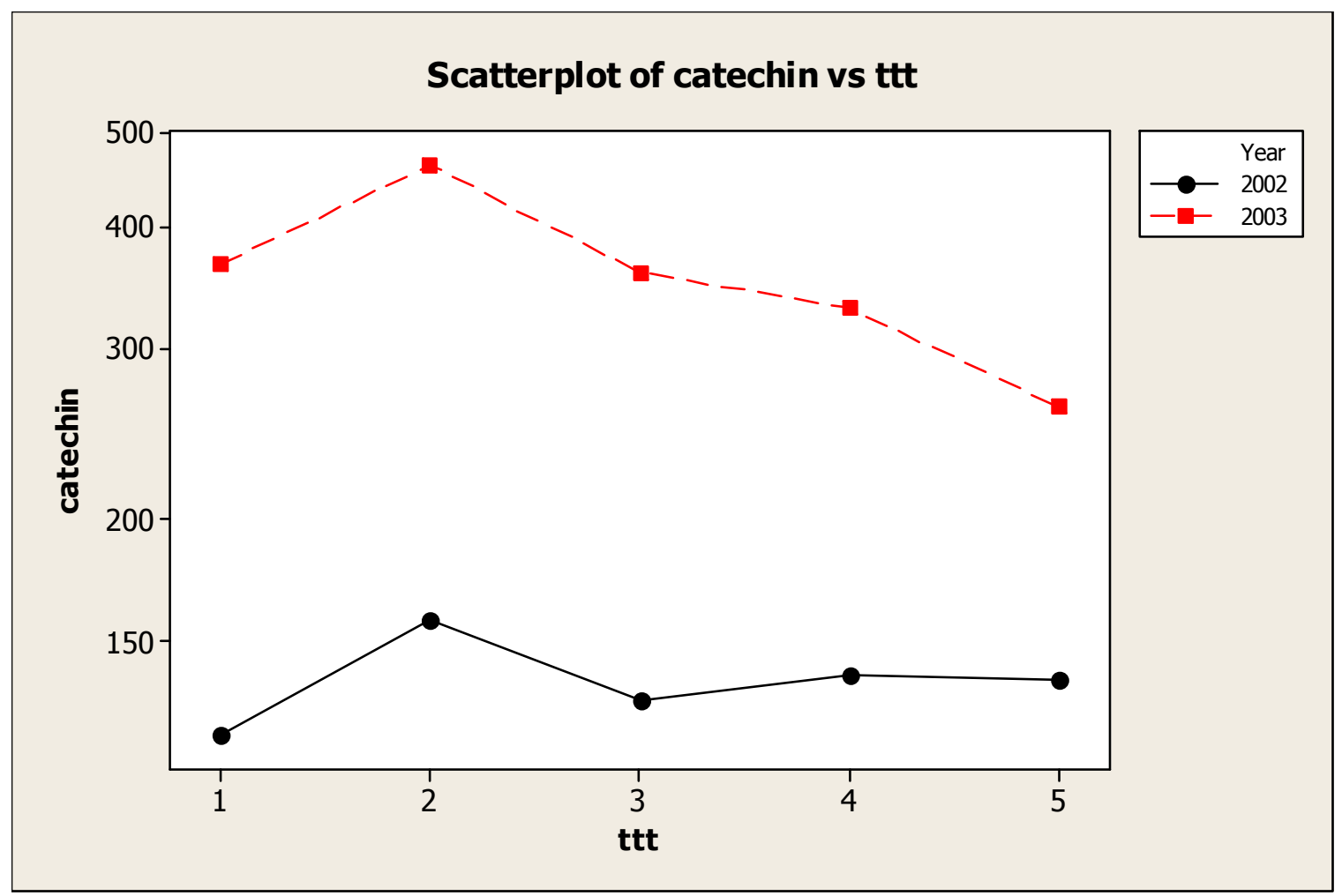


Figure 27, Scatterplot of epicatechin versus ttt based on 2002 and 2003 growing seasons.

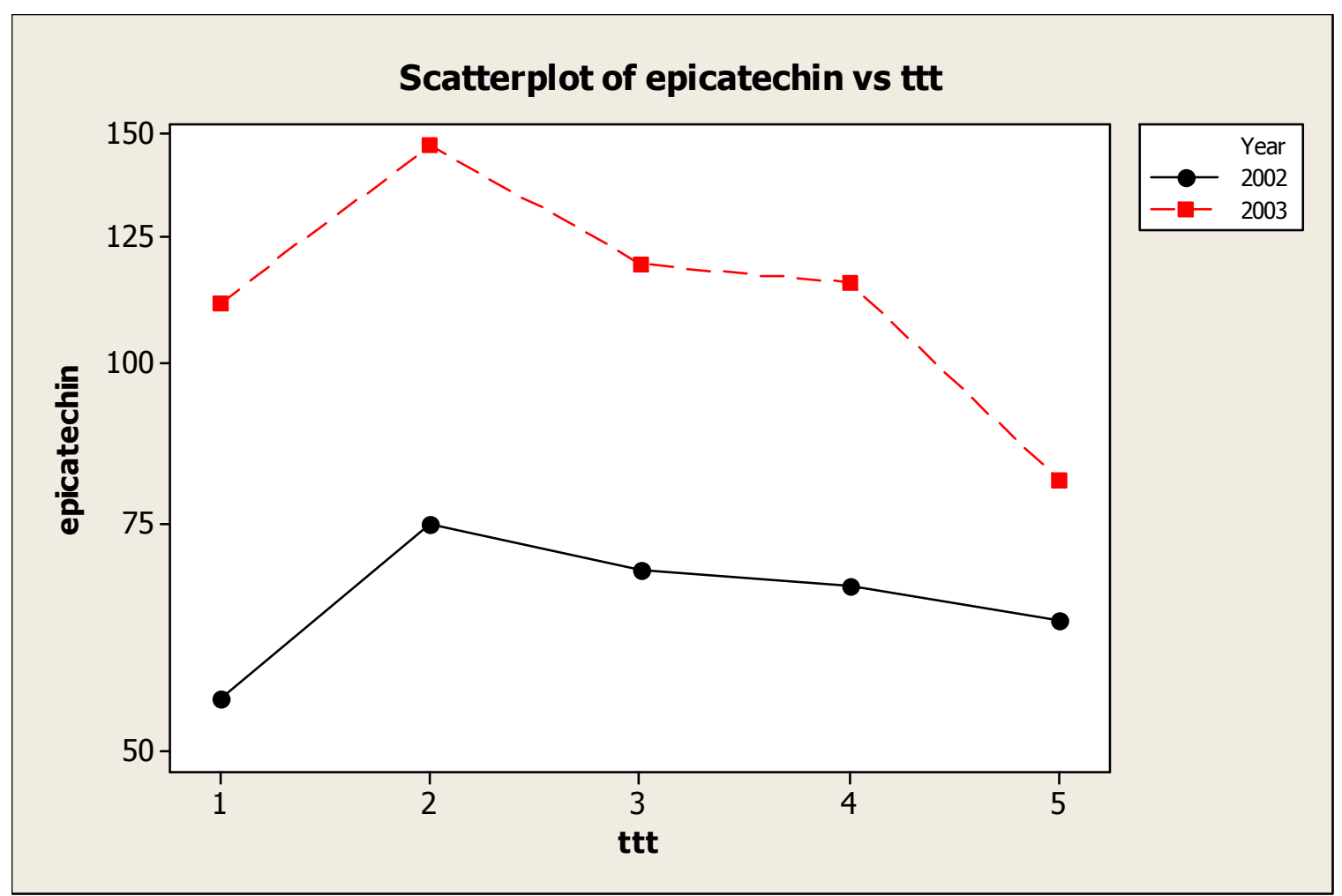


Figure 28, Interaction Plot for polymeric phenols based on 2002 and 2003 growing seasons.

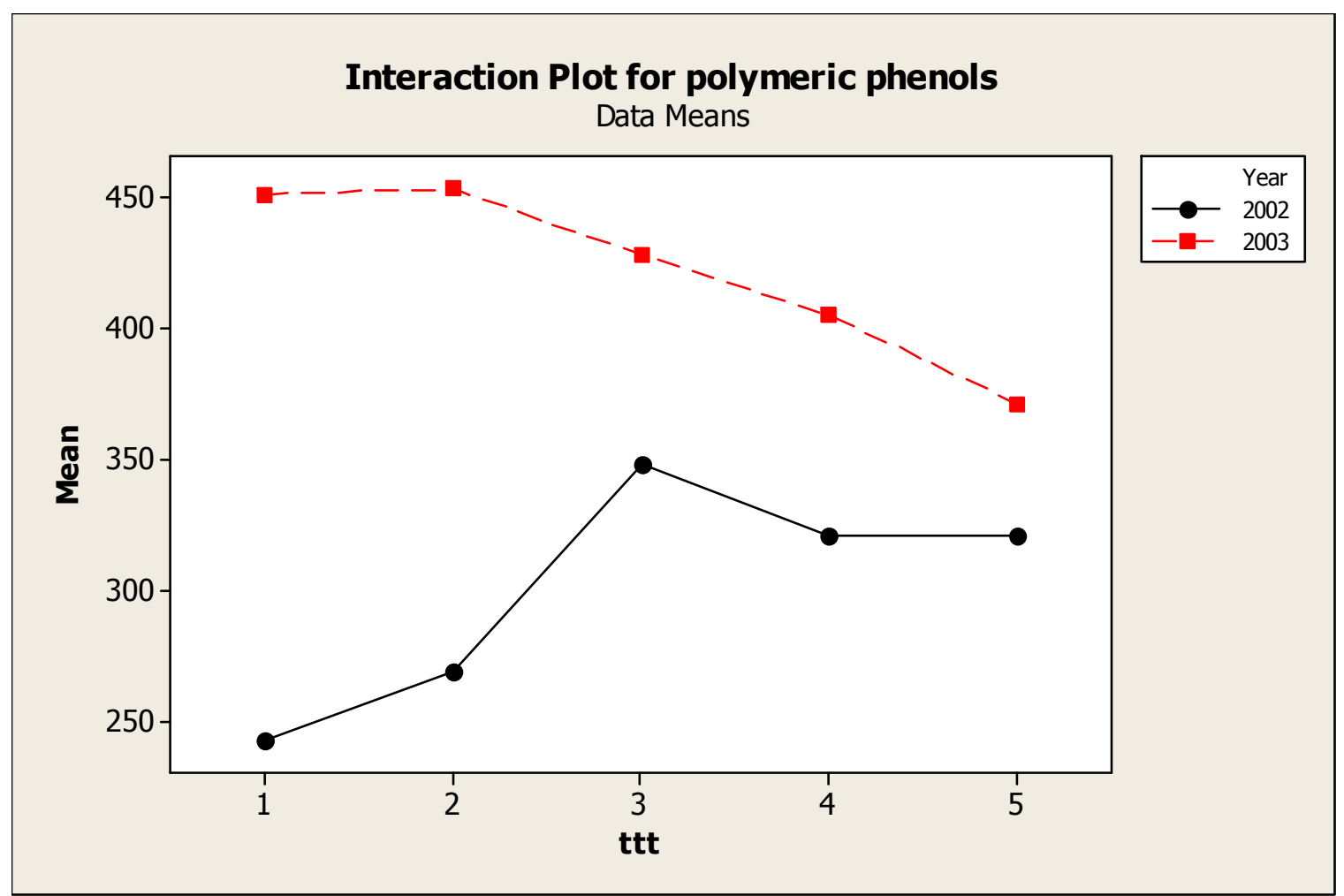


Figure 29, Interaction Plot for caftaric acid based on 2002 and 2003 growing seasons.

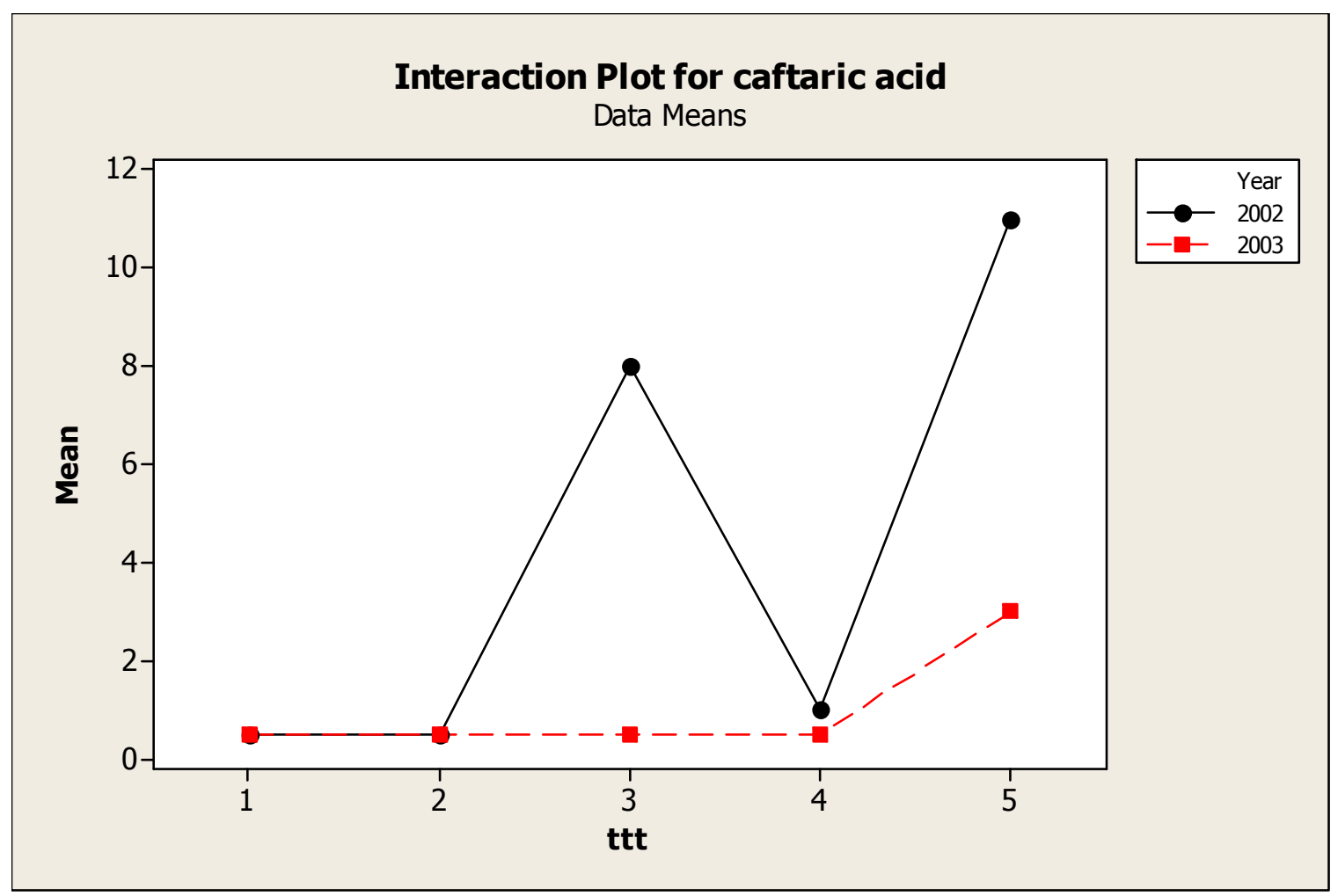


Figure 30, Interaction Plot for Caffeic Acid based on 2002 and 2003 growing seasons.

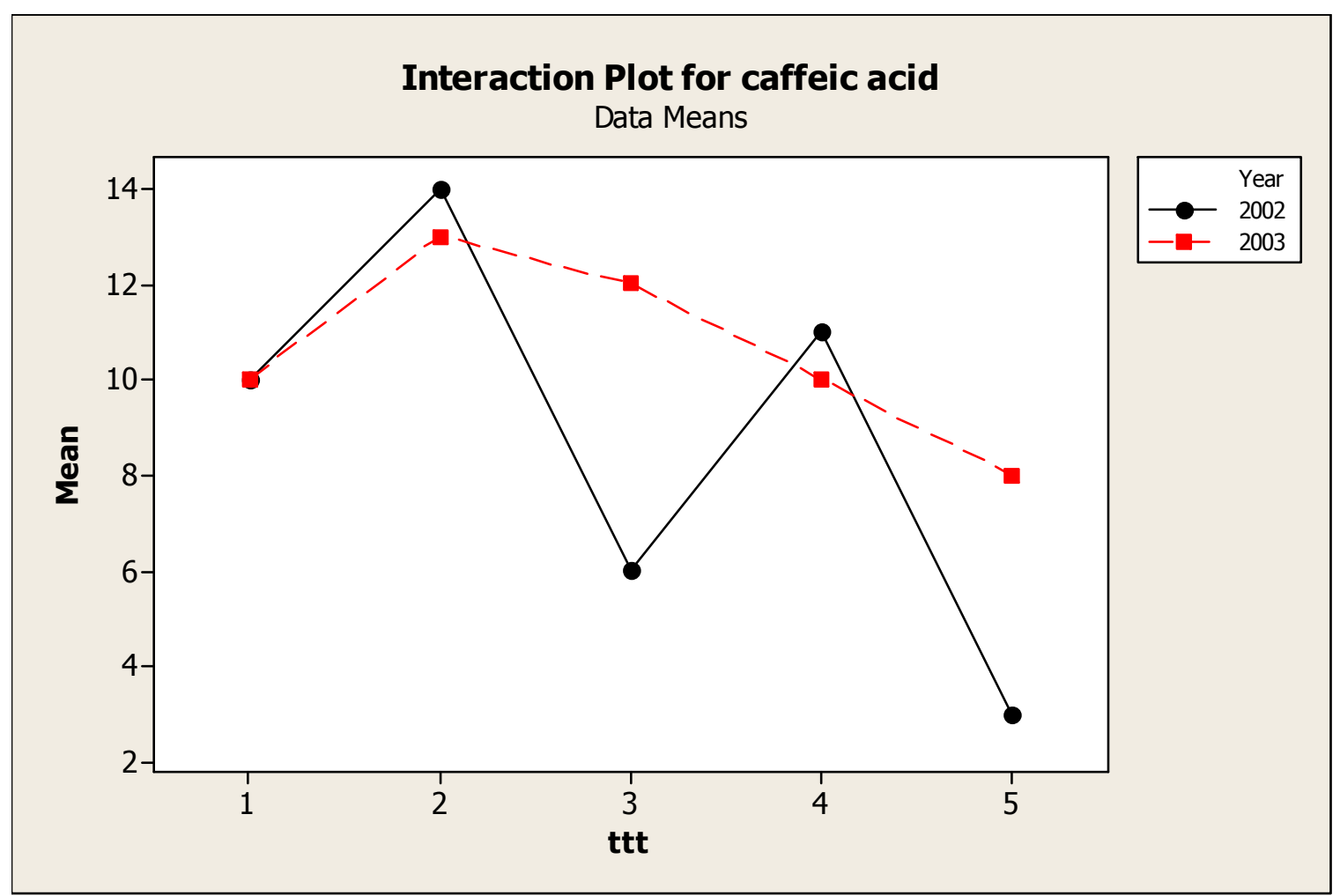


Figure 31, Interaction Plot for quercetin glycosides based on 2002 and 2003 growing seasons.

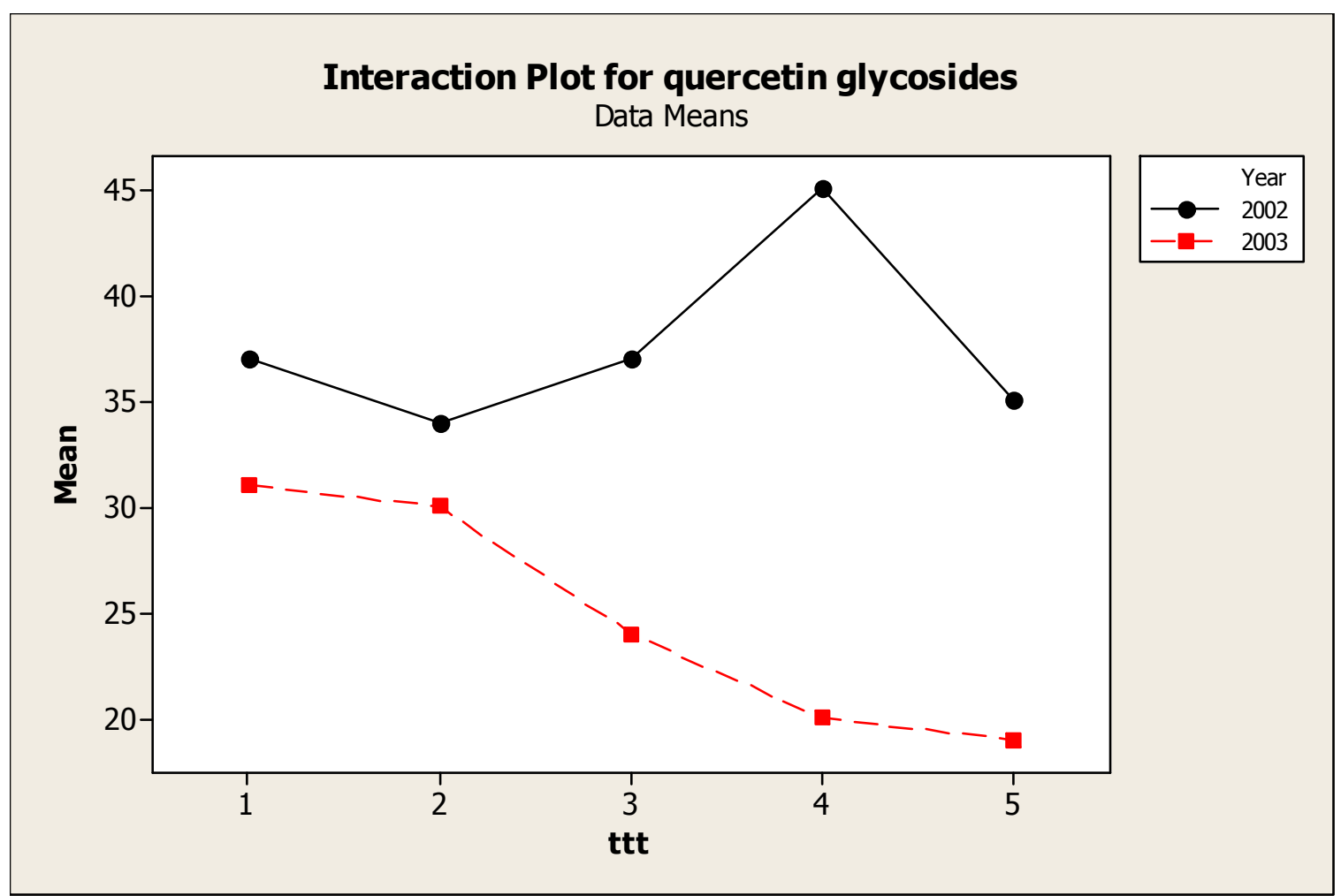


Figure 32, Interaction Plot for quercetin aglycone based on 2002 and 2003 growing seasons.

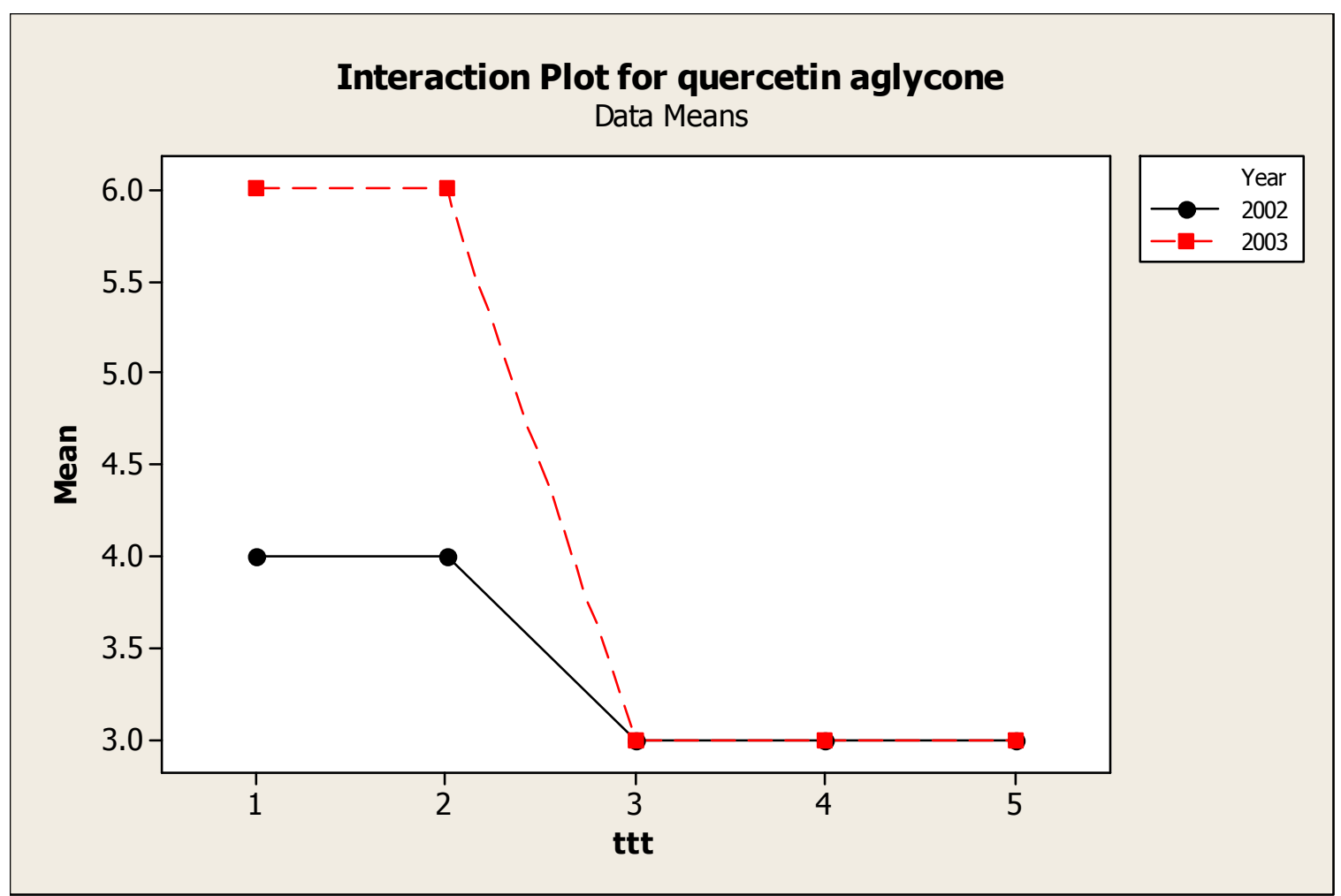


Figure 33, Interaction Plot for malvidin glucoside based on 2002 and 2003 growing season.

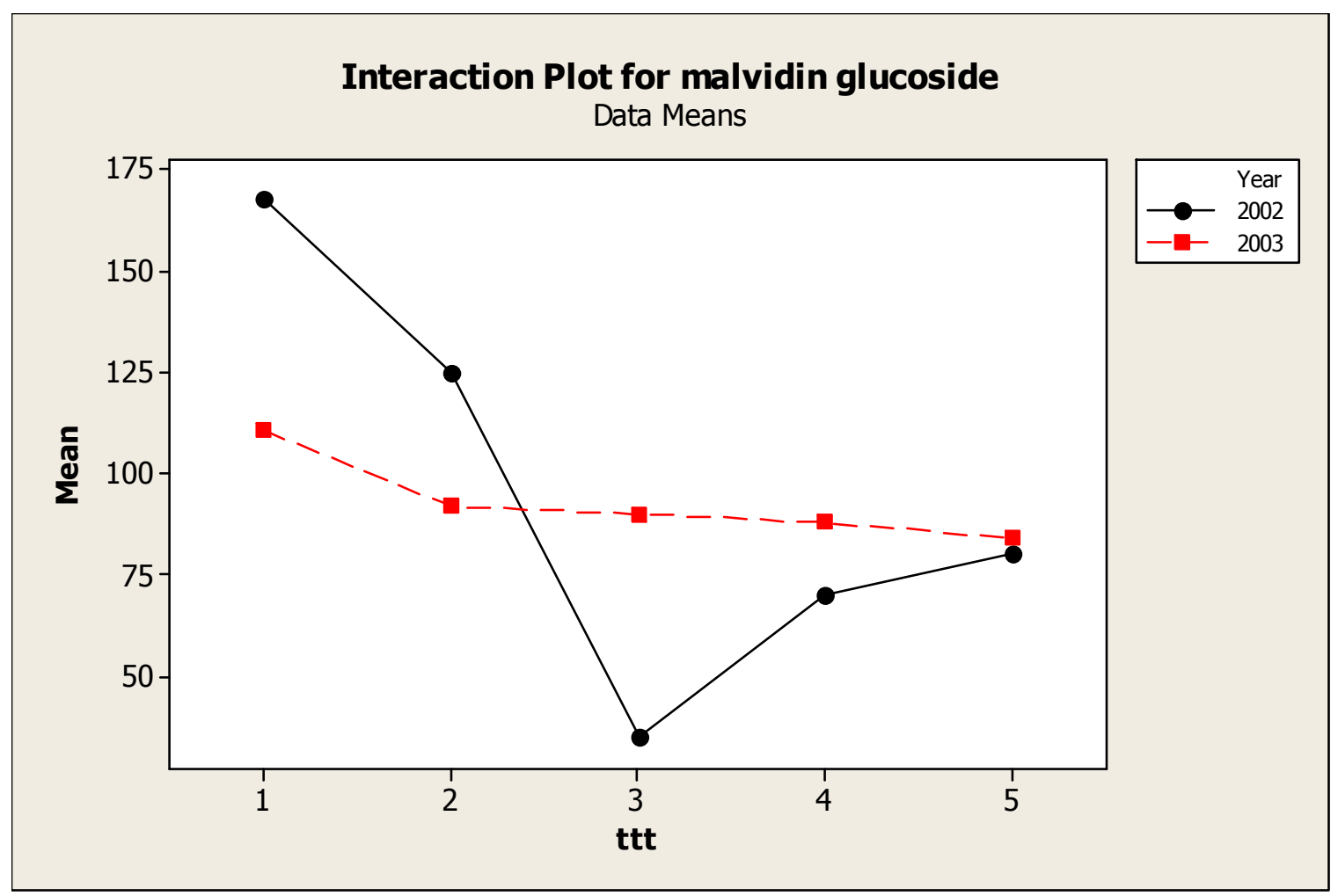


Figure 34, Interaction Plot for polymeric anthocyanins based on 2002 and 2003 growing seasons.

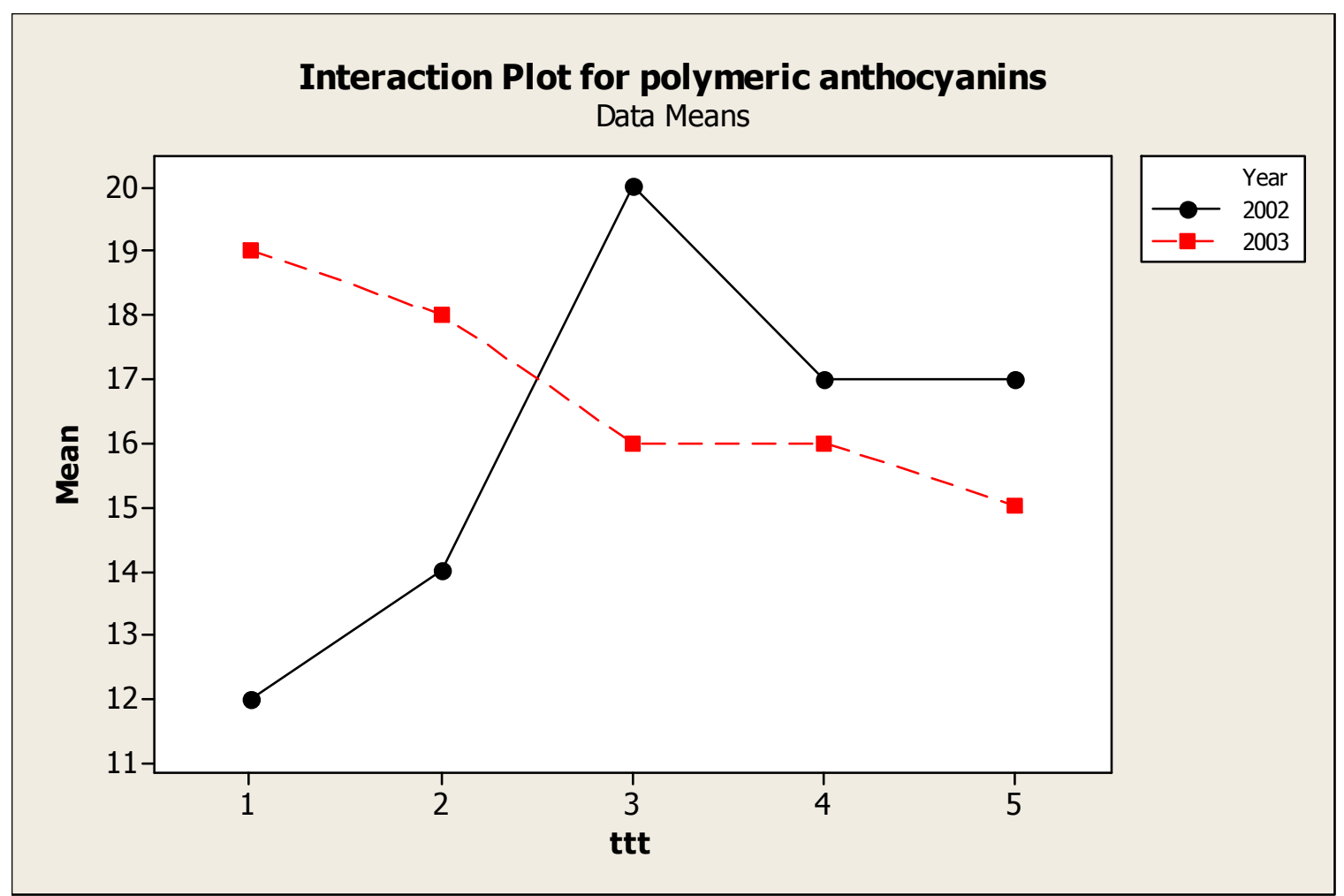


Figure 35, Interaction Plot for total anthocyanins based on 2002 and 2003 growing seasons.

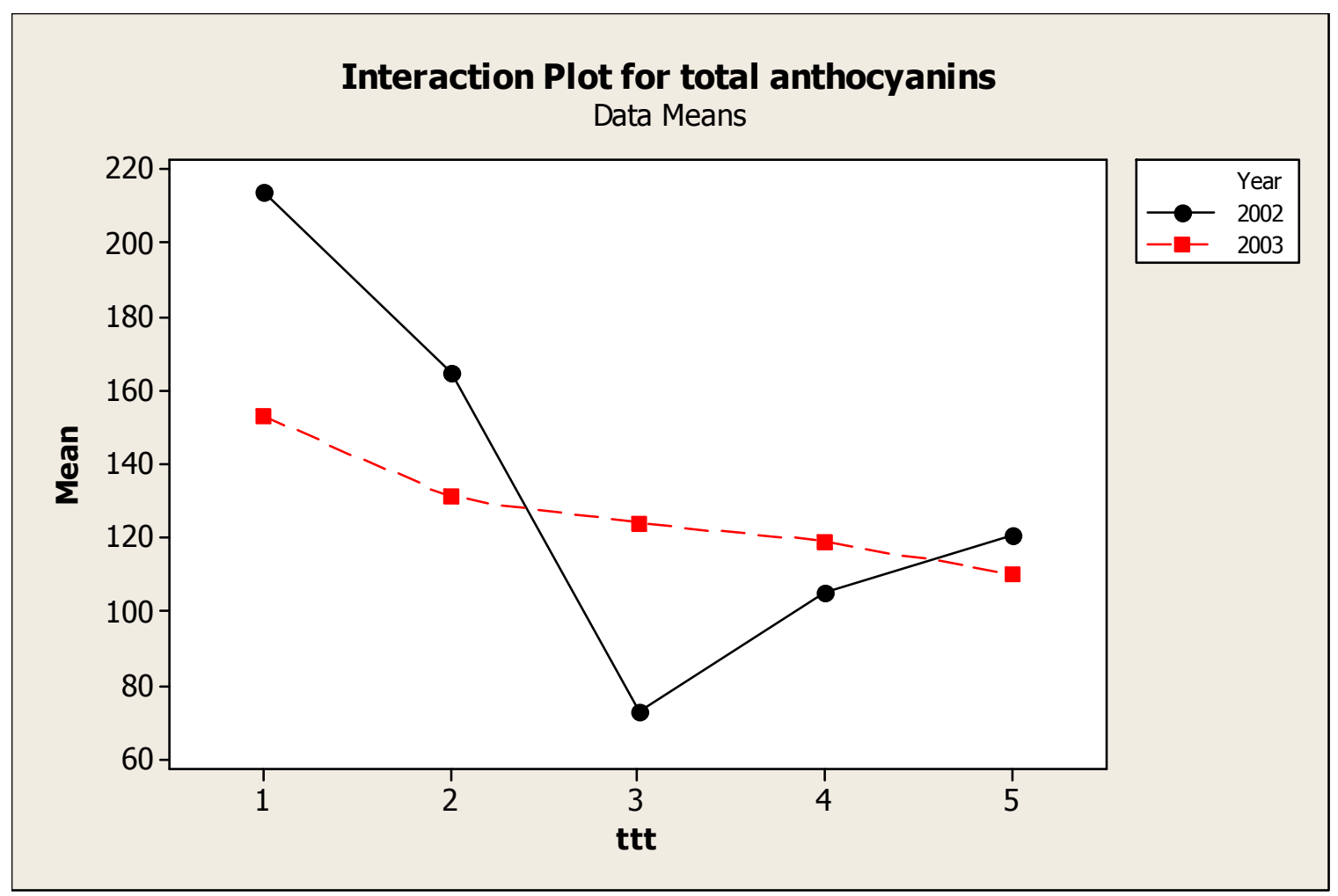


Figure 36, Interaction Plot for monomeric anthocyanins based on 2002 and 2003 growing seasons.

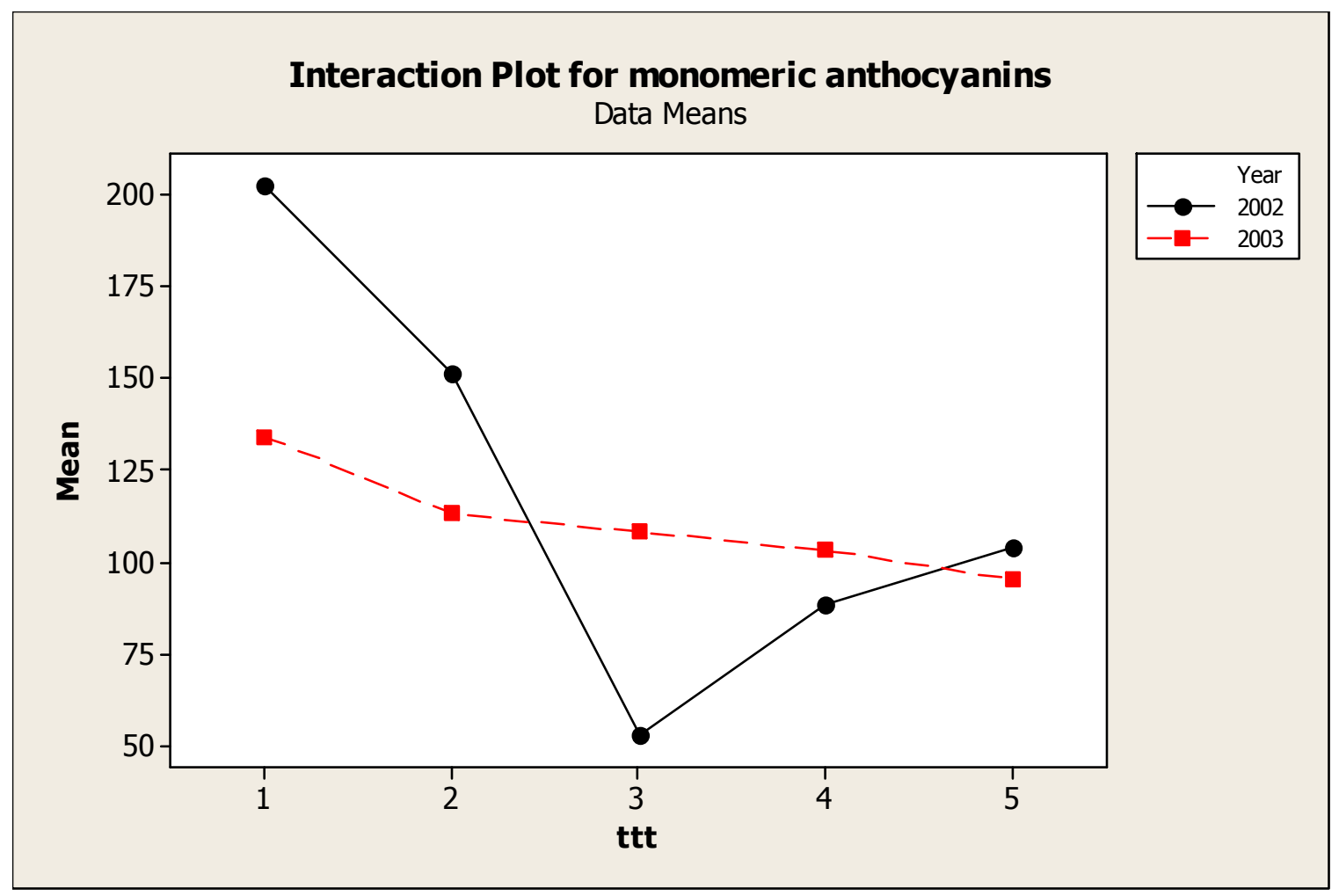


Table 1. Harvest Results.

2002 Growing Season

Harvested 10-12-02

\begin{tabular}{|c|c|c|c|c|c|c|c|c|}
\hline Treatments & & Brix & & pH & & $\underline{\text { TA }}$ & & $\underline{\underline{\text { Actual }}}$ \\
\hline & & & & & & & & \\
\hline Y 1 ton early & & 23.8 & & 3.2 & & 0.78 & & 0.96 \\
\hline YY 1 ton late & & 23.8 & & 3.2 & & 0.85 & & 1.01 \\
\hline & & & & & & & & \\
\hline W 2 ton early & & 22.8 & & 3.5 & & 0.73 & & 1.48 \\
\hline WW 2 ton late & & 24.5 & & 3.3 & & 0.64 & & 1.5 \\
\hline & & & & & & & & \\
\hline P 3 ton early & & 24.2 & & 3.4 & & 0.58 & & 2.08 \\
\hline PP 3 ton late & & 25.6 & & 3.2 & & 0.75 & & 2.45 \\
\hline & & & & & & & \\
\hline B 4 ton early & & 25.2 & & 3.2 & & 0.73 & & 3.39 \\
\hline BB 4 ton late & 24.4 & & 3.3 & & 0.72 & & 2.53 \\
\hline & & & & & & & \\
\hline R 5 ton control & & 24.8 & & 3.3 & & 0.66 & & 3.26 \\
\hline
\end{tabular}

2003 Growing Season

Harvested 9-29-03

\begin{tabular}{|c|c|c|c|c|}
\hline Treatments & Brix & $\mathrm{pH}$ & TA & Tons/Acre \\
\hline Y 1 ton early & 23.4 & 3.21 & 0.855 & 0.69 \\
\hline YY 1 ton late & 24.2 & 3.17 & 0.95 & 0.9 \\
\hline W 2 ton early & 23.5 & 3.19 & 0.65 & 1.45 \\
\hline WW 2 ton late & 24.8 & 3.21 & 0.795 & 1.65 \\
\hline & & & & \\
\hline P 3 ton early & 23.6 & 3.3 & 0.99 & 2.57 \\
\hline PP 3 ton late & 25 & 3.36 & 0.84 & 2.1 \\
\hline B 4 ton early & 25 & 339 & 089 & 236 \\
\hline BB 4 ton late & 25 & 3.41 & 0.8 & 2.64 \\
\hline R 5 ton control & 262 & 337 & (8) & 273 \\
\hline
\end{tabular}


Table 2. Pruning Results.

2002 Growing Season

Pruned 1-13-03

\begin{tabular}{|l|c|l|c|c|c|c|c|}
\hline & $\begin{array}{c}\text { AVG. } \\
\text { Pounds/Shoot }\end{array}$ & & & $\begin{array}{c}\text { AVG. } \\
\text { Pounds/Vine }\end{array}$ & & $\underline{\text { Pounds/Acre }}$ & 单s/Acre \\
\hline Y 1 ton early & 0.178 & & & 3.633 & & 4944.741 & 2.472 \\
\hline YY 1 ton late & 0.134 & & & 2.753 & & 3746.841 & 1.873 \\
\hline & & & & & & & \\
\hline W 2 ton early & 0.141 & & & 2.990 & & 4070.138 & 2.035 \\
\hline WW 2 ton late & 0.135 & & & 2.735 & & 3723.019 & 1.862 \\
\hline & & & & & & & \\
\hline P 3 ton early & 0.133 & & & 2.900 & & 3947.625 & 1.974 \\
\hline PP 3 ton late & 0.121 & & & 2.668 & & 3631.134 & 1.816 \\
\hline & & & & & & & \\
\hline B 4 ton early & 0.139 & & & 2.905 & & 3954.431 & 1.977 \\
\hline BB 4 ton late & 0.122 & & & 2.895 & & 3940.819 & 1.970 \\
\hline & & & & & & & \\
\hline R 5 ton control & 0.127 & & & 2.694 & & 3666.867 & 1.833 \\
\hline
\end{tabular}

2003 Growing Season

Pruned 2-5-04

\begin{tabular}{|c|c|c|c|c|}
\hline & $\begin{array}{c}\text { AVG. } \\
\text { Pounds/Shoot }\end{array}$ & $\begin{array}{c}\text { AVG. } \\
\text { Pounds/Vine }\end{array}$ & Pounds/Acre & Tons/Acre \\
\hline $\mathrm{Y} 1$ ton early & 0.171 & 3.183 & 4332.178 & 2.166 \\
\hline YY 1 ton late & 0.143 & 2.893 & 3937.416 & 1.969 \\
\hline W 2 ton earlv & 0,138 & 2893 & 3937416 & 1969 \\
\hline WW 2 ton late & 0.140 & 2.573 & 3501.816 & 1.751 \\
\hline P 3 ton earlv & 0.132 & 2878 & 3916.997 & 1958 \\
\hline PP 3 ton late & 0.130 & 2.445 & 3328.256 & 1.664 \\
\hline B 4 ton earlv & 0.162 & 3105 & 4226681 & 2113 \\
\hline BB 4 ton late & 0.143 & 3.028 & 4121.184 & 2.061 \\
\hline R 5 ton control & 0.144 & 2.946 & 4010.583 & 2.005 \\
\hline
\end{tabular}


Table 3, Pruning to Fruit ratios 2002

2002 Growing Season

Measured in

Pounds

shoot:cluster prunings/vine:fruit/vine prunings/acre:fruit/acre

\begin{tabular}{|l|l|l|l|l|}
\hline$\underline{\mathrm{Y}}$ & one ton early & $.178: .192$ & $3.63: 1.41$ & $4945: 1916$ \\
\hline $\mathrm{Y} \mathrm{Y}$ & one ton late & $.134: .190$ & $2.75: 1.48$ & $3747: 2015$ \\
\hline$\underline{\mathrm{W}}$ & two ton early & $.141: .156$ & $2.99: 2.18$ & $4070: 2964$ \\
\hline$\underline{\mathrm{W}}$ & two ton late & $.135: .154$ & $2.74: 2.21$ & $3723: 3002$ \\
\hline$\underline{\mathrm{P}}$ & three ton early & $.133: .160$ & $2.90: 3.06$ & $3948: 4162$ \\
\hline $\mathbf{P P}$ & three ton late & $.121: .168$ & $2.67: 3.60$ & $3631: 4904$ \\
\hline$\underline{\mathrm{B}}$ & four ton early & $.139: .185$ & $2.91: 4.98$ & $3954: 6772$ \\
\hline$\underline{\mathrm{BB}}$ & four ton late & $.122: .143$ & $2.90: 3.72$ & $3941: 5067$ \\
\hline$\underline{\mathbf{R}}$ & five ton control & $.127: .160$ & $2.69: 4.79$ & $3667: 6520$ \\
\hline & & & & \\
\hline
\end{tabular}

shoot:cluster prunings/vine:fruit/vine prunings/acre:fruit/acre

\begin{tabular}{|l|l|c|c|c|}
\hline$\underline{Y}$ & one ton early & 1 to 1.07 & 1 to .39 & 1 to .39 \\
\hline$\underline{Y} \mathbf{Y}$ & one ton late & 1 to 1.42 & 1 to .54 & 1 to .54 \\
\hline$\underline{\mathbf{W}}$ & two ton early & 1 to 1.11 & 1 to .73 & 1 to .73 \\
\hline$\underline{\mathbf{W}}$ & two ton late & 1 to 1.14 & 1 to .81 & 1 to .81 \\
\hline$\underline{P}$ & three ton early & 1 to 1.20 & 1 to 1.01 & 1 to 1.35 \\
\hline$\underline{P}$ & three ton late & 1 to 1.39 & 1 to 1.35 & 1 to 1.71 \\
\hline$\underline{B}$ & four ton early & 1 to 1.33 & 1 to 1.71 & 1 to 1.29 \\
\hline$\underline{\mathrm{R}}$ & four ton late & 1 to 1.17 & 1 to 1.28 & 1 to 1.78 \\
\hline & five ton control & 1 to 1.26 & 1 to 1.78 & \\
\hline
\end{tabular}


Table 4, Pruning to Fruit ratios 2003

2003 Growing Season

Measured in

Pounds

shoot:cluster prunings/vine:fruit/vine prunings/acre:fruit/acre

\begin{tabular}{|l|l|l|l|l|}
\hline$\underline{Y}$ & one ton early & $0.171: 0.125$ & $3.18: 1.01$ & $4328: 1375$ \\
\hline$\underline{Y} \mathbf{Y}$ & one ton late & $0.143: 0.143$ & $2.89: 1.32$ & $3933: 1797$ \\
\hline$\underline{W}$ & two ton early & $0.138: 0.146$ & $2.89: 2.14$ & $3933: 2913$ \\
\hline$\underline{W} \mathbf{t}$ & two ton late & $0.140: 0.139$ & $2.57: 2.43$ & $3498: 3307$ \\
\hline$\underline{P}$ & three ton early & $0.132: 0.154$ & $2.88: 3.78$ & $3920: 5145$ \\
\hline$\underline{P P}$ & three ton late & $0.130: 0.135$ & $2.45: 3.08$ & $3334: 4192$ \\
\hline$\underline{B}$ & four ton early & $0.162: 0.126$ & $3.11: 3.47$ & $4233: 4723$ \\
\hline$\underline{B}$ & four ton late & $0.143: 0.127$ & $3.03: 3.87$ & $4124: 5267$ \\
\hline$\underline{R}$ & five ton control & $0.144: 0.124$ & $2.95: 4.01$ & $4015: 5458$ \\
\hline & & & & \\
\hline
\end{tabular}

shoot:cluster prunings/vine:fruit/vine prunings/acre:fruit/acre

\begin{tabular}{|c|c|c|c|c|}
\hline$\underline{Y}$ & one ton early & 1 to .73 & 1 to .32 & 1 to .32 \\
\hline$\underline{Y Y}$ & one ton late & 1 to 1 & 1 to .46 & 1 to .46 \\
\hline$\underline{\underline{\mathbf{W}}}$ & two ton early & 1 to 1.06 & 1 to .74 & 1 to .74 \\
\hline WW & two ton late & 1 to .99 & 1 to .95 & 1 to .95 \\
\hline $\bar{P}$ & three ton early & 1 to 1.17 & 1 to 1.31 & 1 to 1.31 \\
\hline PP & three ton late & 1 to 1.04 & 1 to 1.26 & 1 to 1.26 \\
\hline B & four ton early & 1 to .78 & 1 to 1.12 & 1 to 1.12 \\
\hline BB & four ton late & 1 to .89 & 1 to 1.28 & 1 to 1.28 \\
\hline$\underline{\mathbf{R}}$ & five ton control & 1 to .86 & 1 to 1.36 & 1 to 1.36 \\
\hline
\end{tabular}




\section{Literature Cited}

1. Bacon T.; Byrne D.H. Chilling Accumulation: its Importance and Estimation. Website accessed (July 2009). http://aggiehorticulture.tamu.edu/stonefruit/chillacc.htm

2. Bates, T.; Morris, J. (Jan. 2003). Effect of Pruning, Training, Thinning, and Shoot Positioning on the Yield and Quality of 'Concord' Grapevines. Lake Erie Grape Research. Cornell University, New York State Agricultural Experimental Station. http://lergp.cce.cornell.edu/Bates/Reports/2004(a)BatesJuiceQualityFinal.pdf

3. Bearden A.N.; Bowers K.; Kasimatis A.N. (1997). In Wine Grape Varieties In The North Coast Counties Of California. Agricultural Sciences Publications, Richmond, CA.

4. Beelman, R. (1982). Development and Utilization of Starter Cultures to Induce Malolactic Fermentation in Red Table Wines. In A.D. Webb Grape Wine Symposium Proceedings of the University of California (Davis) pp. 109-117.

5. Bell,P. (1997). Philosophy of Wine Making. Cornell University, Oct. 2002. http:/www.FS430\%Philosophy\%20ofwine\%20making.htm

6. Boulton, R.B.; Singleton, V.L.; Bisson, L.F.; Kunkee, R.E. (1998). In Principles and Practices of Winemaking, Aspen Publishers, Inc. Gaitherburg, Maryland: 604 p.

7. Bravado, B.; Cohen, S.; Hepner, Y.; Loinger, C.; Tabacman, H. (1985). Effect of Crop Level and Crop Load on Growth, Yield, Must and Wine Composition, and Quality of Cabernet Sauvignon. American Journal of Enology and Viticulture. 36: (2)125-131.

8. Burns, G. (2009). Red Wine Phenolic Report - ETS Laboratories. Website accessed (October 2009). http://www.etslabs.com/display.aspx?catid=48,50,58\&pageid-74

9. Caldwell J.; Melka P. (1998). A Concise Guide To Wine Grape Clones For The Professionals. John Caldwell Viticultural Services, Napa, CA.

10. Coombe, B. G.; Dry P.R. (1988). In Viticulture. Underdale, SA, Winetitles, Adelaide: 1:211 p, 2:376 P. 
11. Dharmadhikari, M. (2000). Vineyard and Vintage View. Grape Newsletter. March/April 2000.

12. Duame, J. (undated). Home Page. http://www.homebeerwinecheese.com/crushtimedecisions.html

13. Henick-Kling, T. (1995). Control of Malo-lactic Fermentation in Wine : Energetics, Flavor Modification and Methods of Starter Culture Preparation. Journal of Applied Bacteriology Symposium Supplement. 79:298-378.

14. Howell, S.G. (1999). Sustainable Grape Productivity and the Growth-Yield Relationship. Presented at the Annual Meeting of the American Society for Enology and Viticulture, Reno, Nevada.

15. Kliewer, W. M.; Lider, L. A. (1967). Concentration Tartaric Acid and Malic Acids and Their Salts in Vitis vinifera Grapes. American Journal of Enology and Viticulture. 18: (1)42-54.

16. Maicas, S.; Gil, J.; Isabel, P.; Ferrer, S. (1999). Improvement of Volatile Composition of Wines by Controlled Addition of Malolactic Bacteria. Food Research International. 32:491-496.

17. Mc Intyre, G. N.; Kliewer, W. M.; Lider, L. A. (1987). Some Limitations of the Degree Day System as Used in Viticulture in California. American Jounal of Enology and Viticulture. 38(2)128-132.

18. Melsom, Michael. Merumwines. Website accessed (Jan. 2004). http://www.merum.com.au/files/CorporateProfileWeb.pdf.

19. Montecalvo, J.; Niku, S. (2001). In Methods of Wine Analysis. Food Science and Nutrition Department, California Polytechnic State University: pp. 24-40.

20. Mullins, M. G.; Bouquet, E.; Williams, L. E. (1992). In Biology of the Grapevine, Cambridge, United Kingdom Cambridge University Press: 239 p.

21. Peynaud, E. (1984). In Knowing and Making Wine. John Wiley \& Sons, Inc. New York, NY: $174 \mathrm{p}$.

22. Pongrancz D.P. (1983). In Rootstocks For Grape-vines, David Philip Publisher, Cape Province, South Africa. 
23. Price, S.F.; Reynolds, A.G.; Wardle, D.A.; Watson, B.T. (1994). Fruit Environment and Crop Level Effects on Pinot noir. I. Vine Performance and Fruit Composition in British Columbia. American Journal of Enology and Viticulture. 45: (4)452-459.

24. Priewe, Jens (1999). In Wine From Grape to Glass. Abbelille Press, New York, NY: 256 p.

25. Riberequ-Gayon, P.; Dubourdieu, Glories, Y.; Maujean, A. (2000). In Handbook of Enology John Wiley \& Sons Ltd. West Sussex, England. 2:404 p.

26. Robinson J. (1986). In Vines, Grapes And Wines, Mitchell Beazley.

27. Robinson M.; Smart R. (1991). In Sunlight Into Wine, Winetitles, Underdale, Australia.

28. Vasquez, S.; Fidelibus, M. (2008). Winter Chilling Requirements for Grapes. Website accessed (July 2009). http://westernfarmpress.com/grapes/winter-chilling $\underline{1210 /}$

29. Walter-Peterson, Hans. (Dec. 2003). Results From Mechanical Thinning Trials. Grape Cultural Practices. The Lake Erie Regional Grape Program.

30. Winkler A.J.; Cook J.A.; Kliewer W.M.; Lider L.A. (1962). In General Viticulture, University of California Press, Berkeley and Los Angeles, CA.

31. Zoecklin, B. W.; Fugelsang, K. C.; Gump, B. H.; Nury, F. S. (1990). In Production Wine Analysis, Van Nostrand Reinhold, New York, NY: 475 p.

32. Zoecklin, B.W.; Fugelsang, K.C.; Gump, B.H.; Nury, F.S. (1999). Wine Analysis and Production. Aspen Publication, Maryland: 
APPENDICES 


\section{Appendix A}

\section{Wine Phenolic Analysis for 2002 growing season}

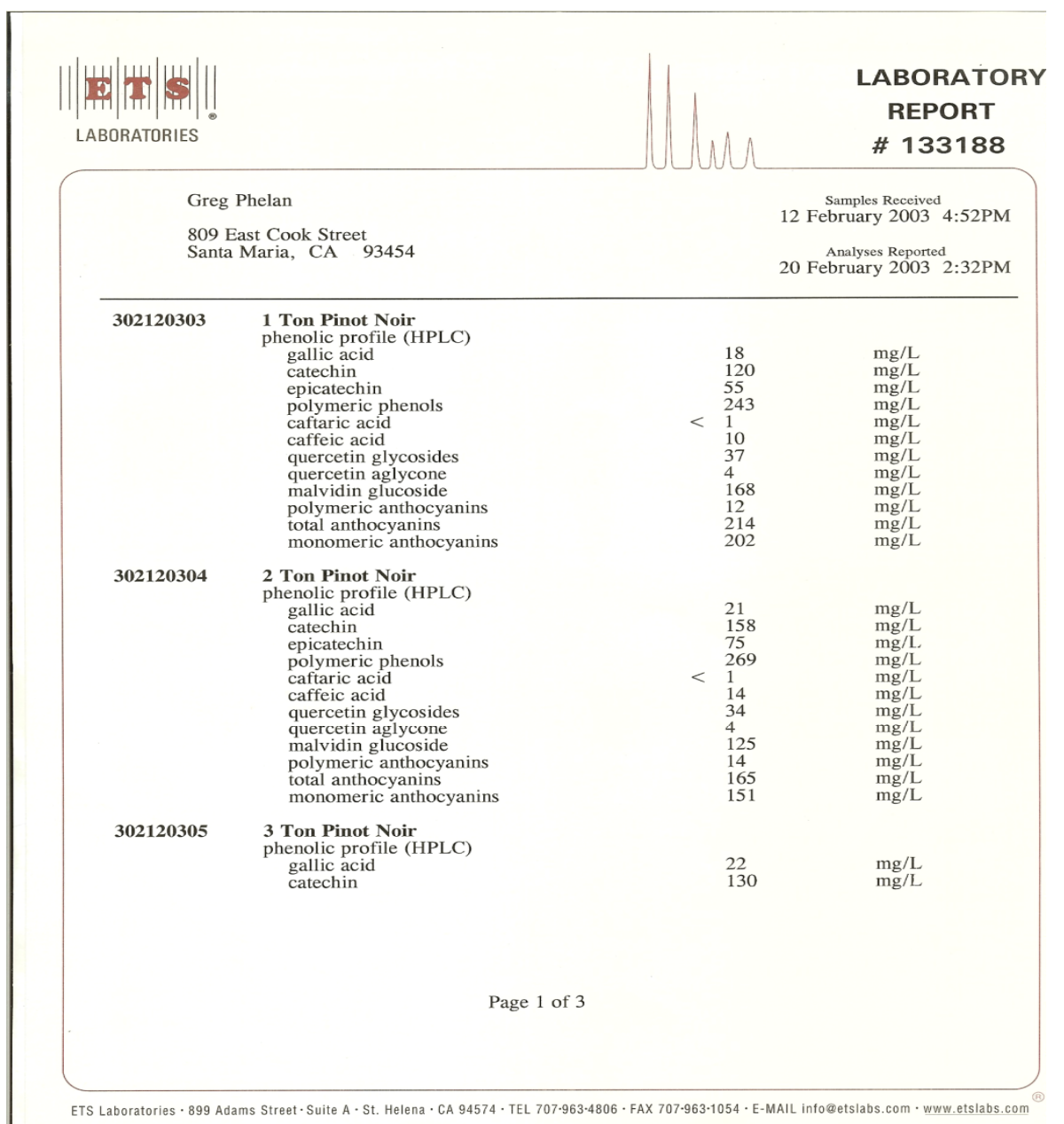




\section{Appendix A cont.}

\section{Wine Phenolic Analysis for 2002 growing season}

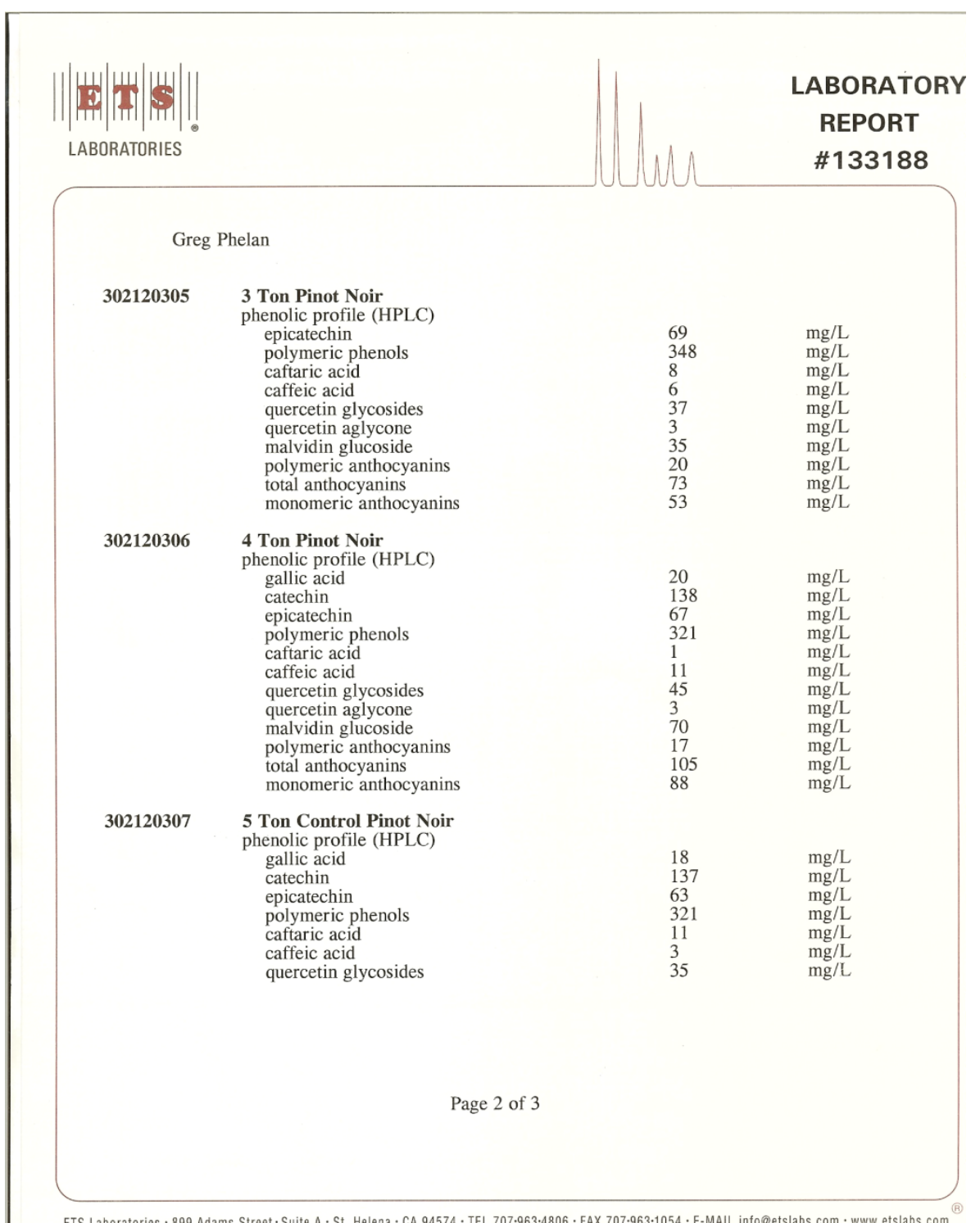

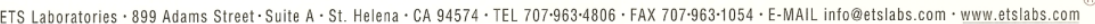




\section{Appendix A cont.}

Wine Phenolic Analysis 2002 growing season

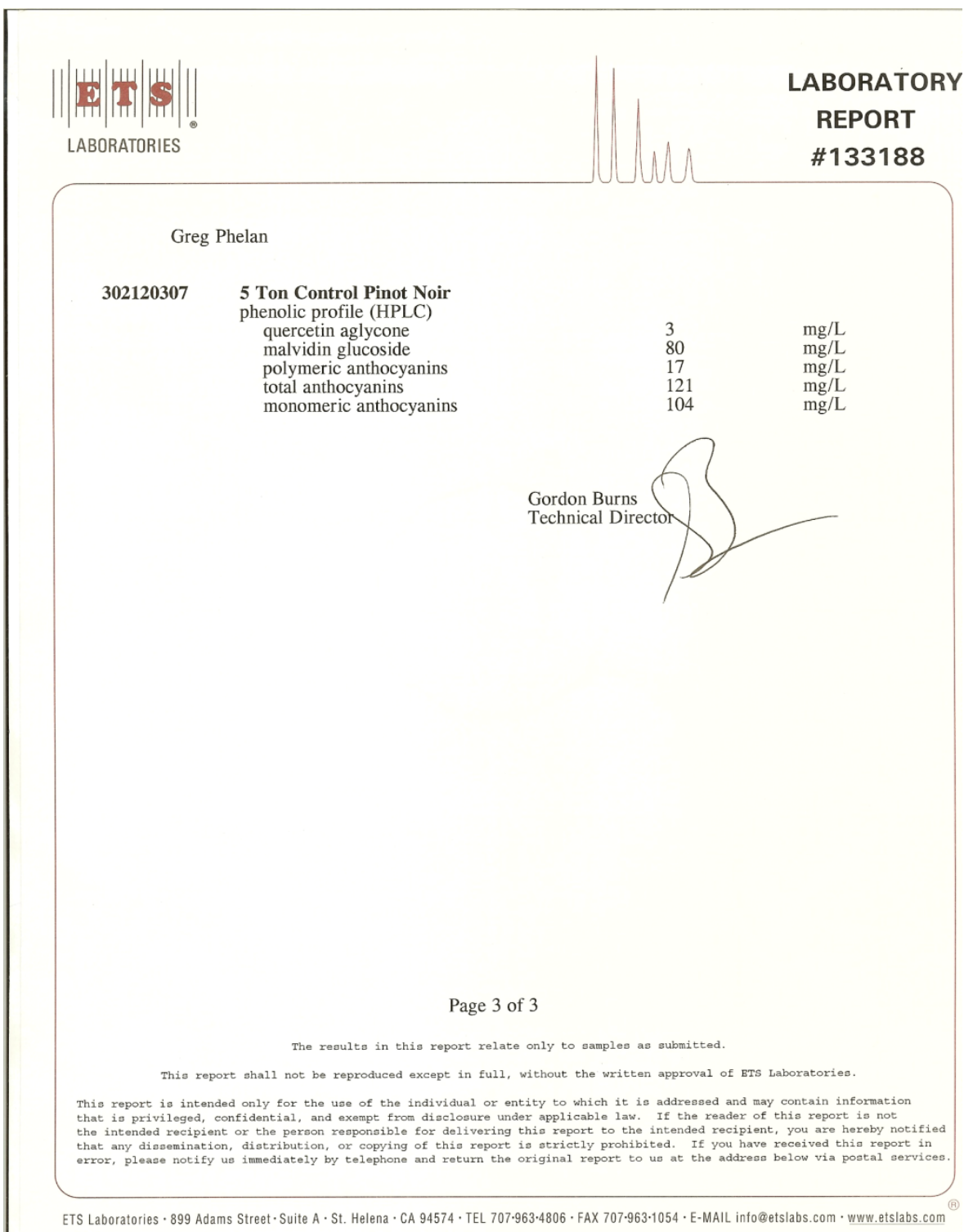




\section{Appendix B}

Wine Analysis 2002 growing season 1 ton treatment compared to 5 ton control

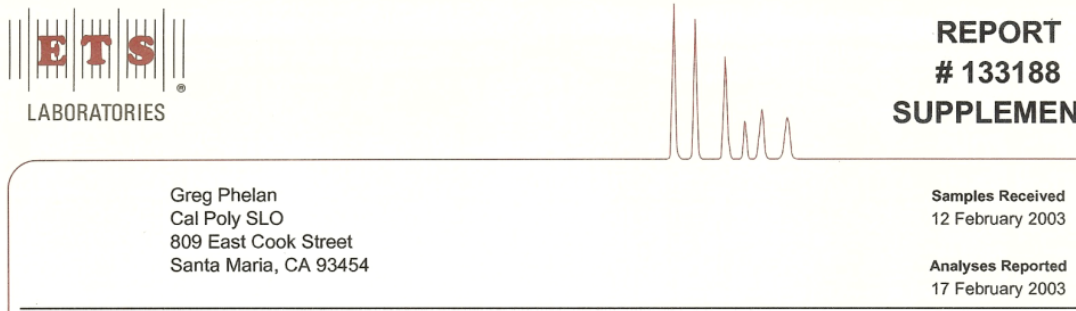

$302120303 \quad$ PN 1 ton gallic acid

polymeric

anthocyanins
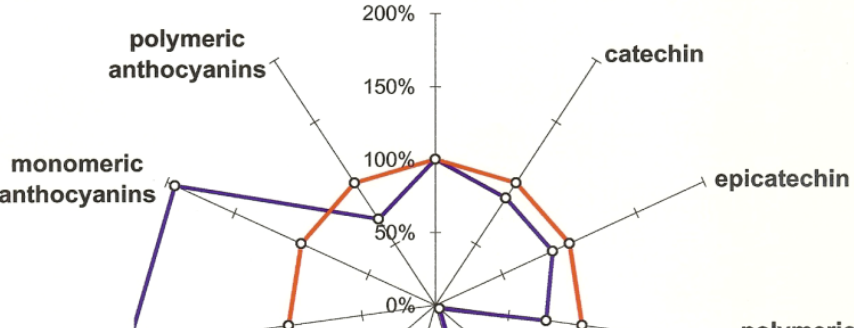

malvidin 5

polymeric
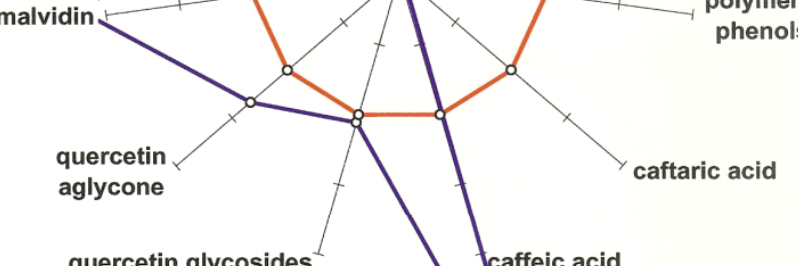

quercetin glycosides $\quad$ tcaffeic acid

$\multimap-$ PN 1 ton $\quad-$ - PN 5 ton

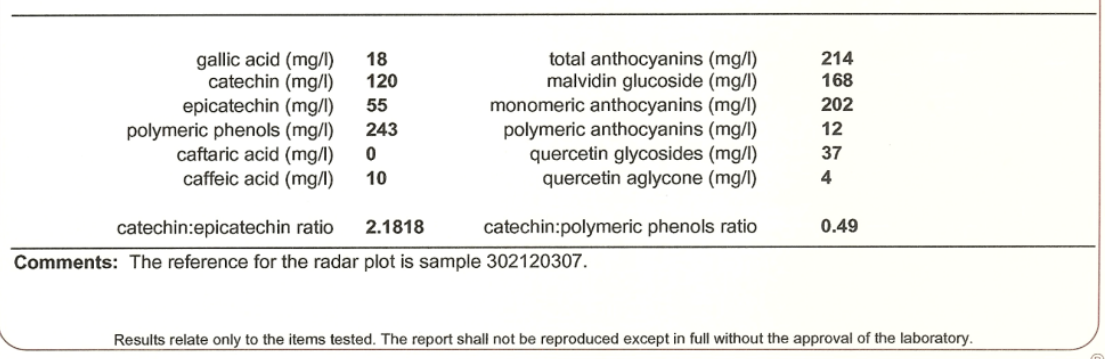

ETS Laboratories · 899 Adams Street·Suite A -St. Helena · CA 94574 · TEL 707-963-4806 · FAX 707-963·1054 · E-MAIL info@etslabs.com · www.etslabs.com 


\section{Appendix C}

Wine Analysis 2002 growing season 2 ton treatment compared to 5 ton control

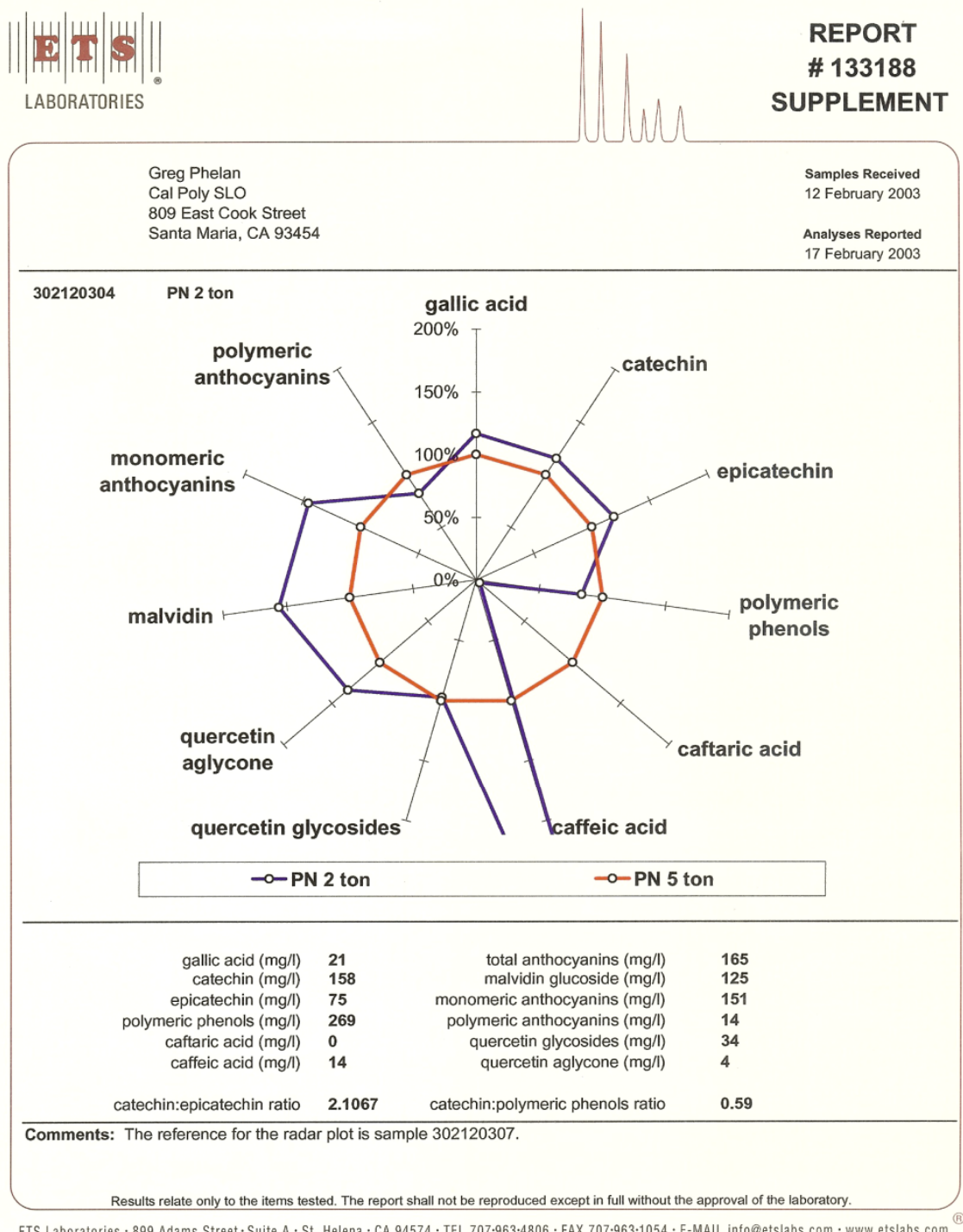




\section{Appendix D}

Wine Analysis 2002 growing season 3 ton treatment compared to 5 ton control

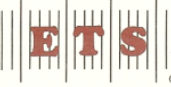

LABORATORIES

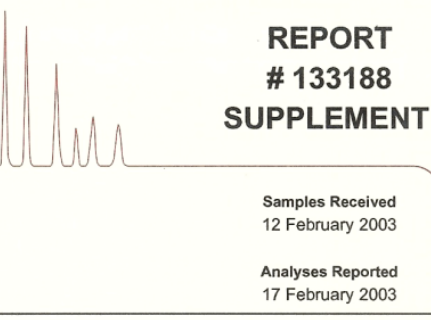

302120305

Cal Poly SLO
809 East Cook Stree

Santa Maria, CA 93454

17 February 2003

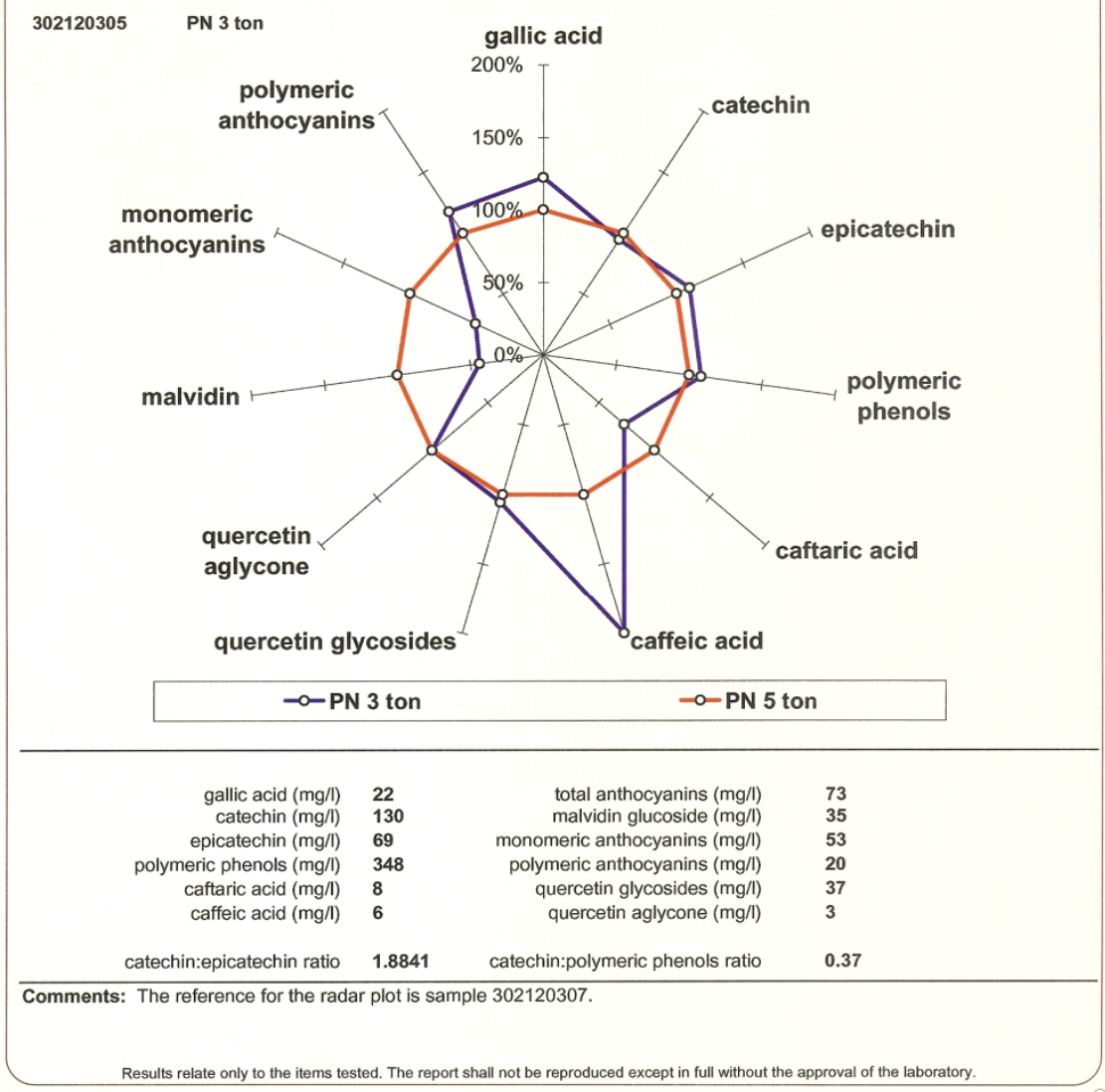

ETS Laboratories · 899 Adams Street · Suite A · St. Helena · CA 94574 · TEL 707-963·4806 · FAX 707-963·1054 · E-MAlL info@etslabs.com · www.etslabs.com 


\section{Appendix E}

Wine Analysis 2002 growing season 4 ton treatment compared to 5 ton control

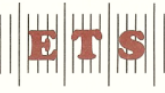

LABORATORIES

REPORT

\# 133188

SUPPLEMENT

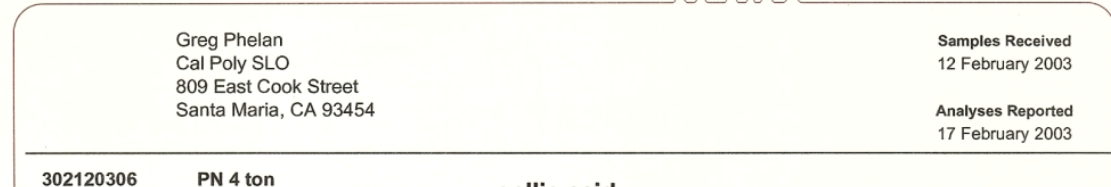

302120306

gallic acid 4 ton

polymeric anthocyanins anthocyanins
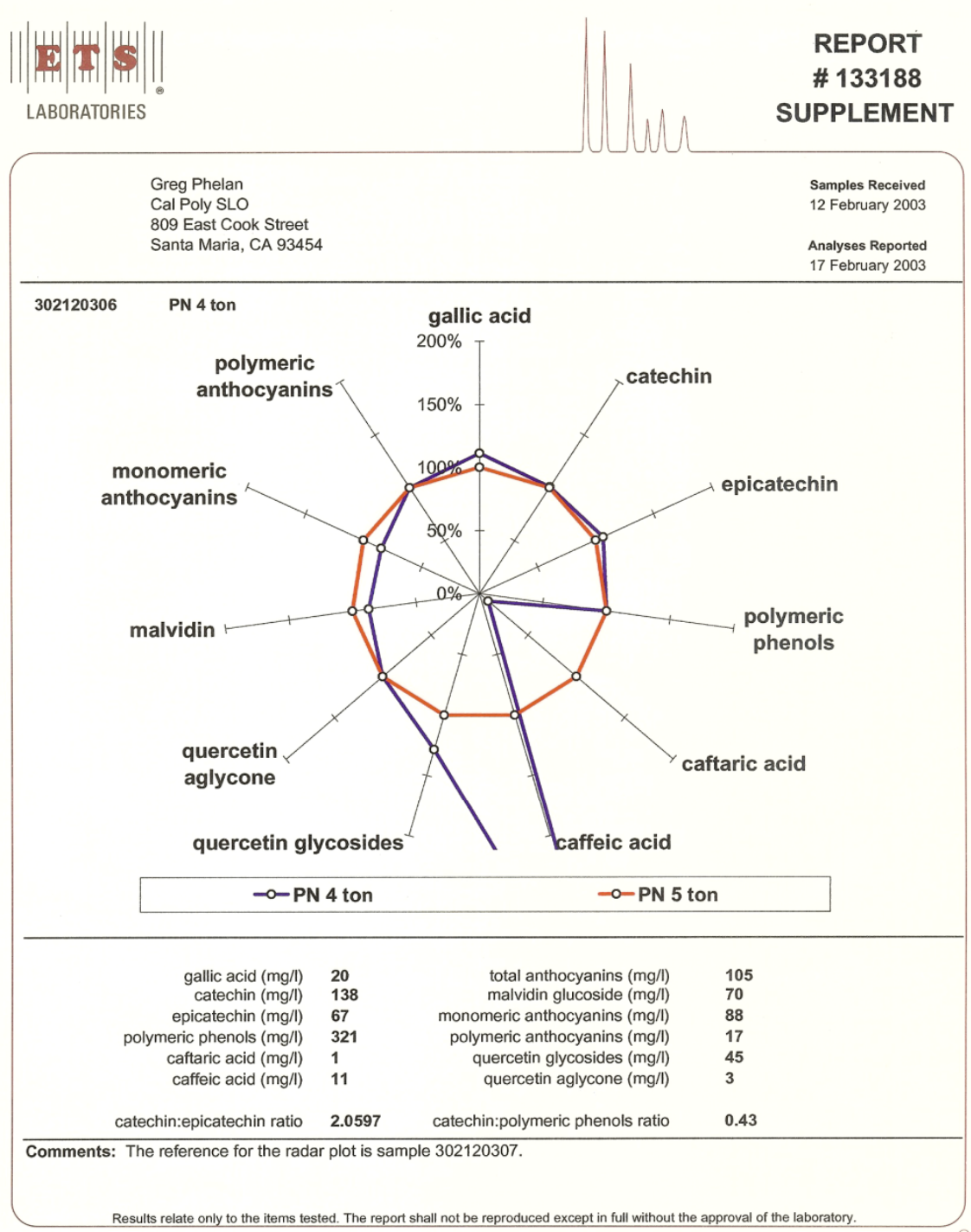

ETS Laboratories· 899 Adams Street·Suite A·St. Helena·CA 94574· TEL 707-963·4806·FAX 707-963·1054·E-MAlL info@etslabs.com·www.etslabs.com 


\section{Appendix F}

\section{Wine Analysis 2002 growing season 5 ton control}

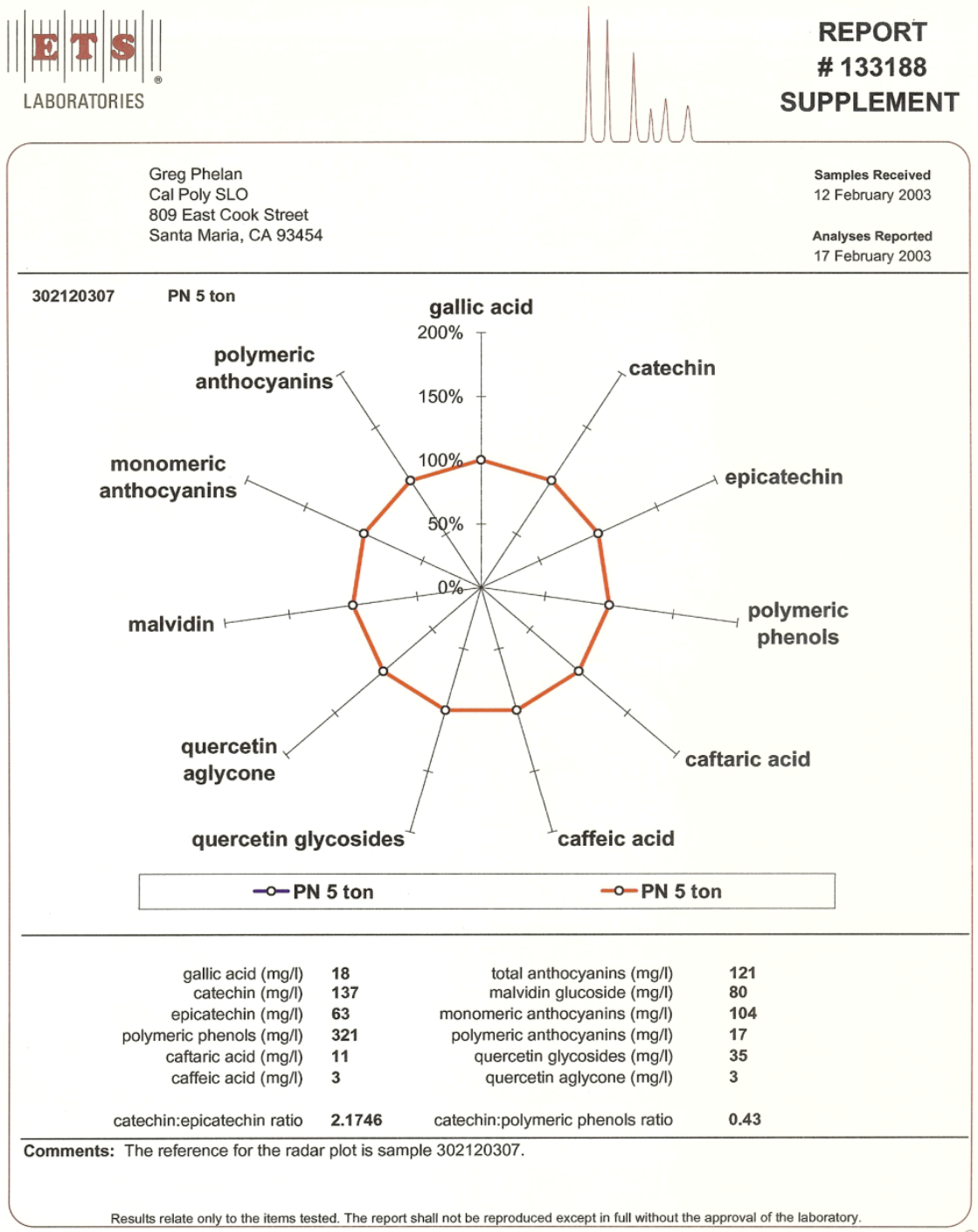

ETS Laboratories · 899 Adams Street· Suite A.St. Helena · CA 94574 · TEL 707-963·4806 · FAX 707-963·1054 · E-MAlL info@etslabs.com · www.etslabs.com 


\section{Appendix G}

Wine Phenolic Analysis 2003 growing season

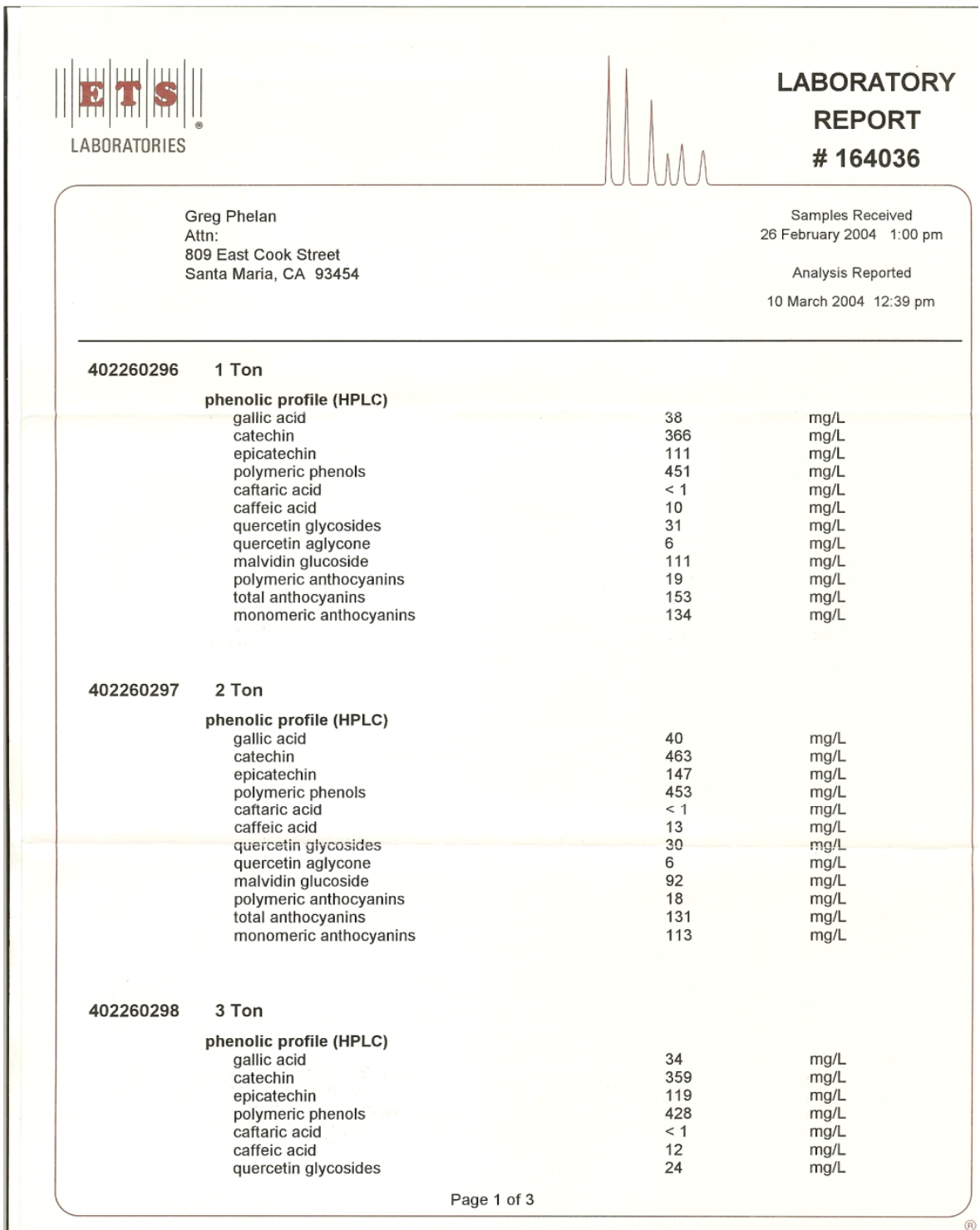

ETS Laboratories · 899 Adams Street - Suite A · St. Helena · CA 94574 · TEL 707-963·4806 · FAX 707-963·1054 · E-MAIL info@etslabs.com · www.etslabs.com 


\section{Appendix G cont.}

Wine Phenolic Analysis 2003 growing season

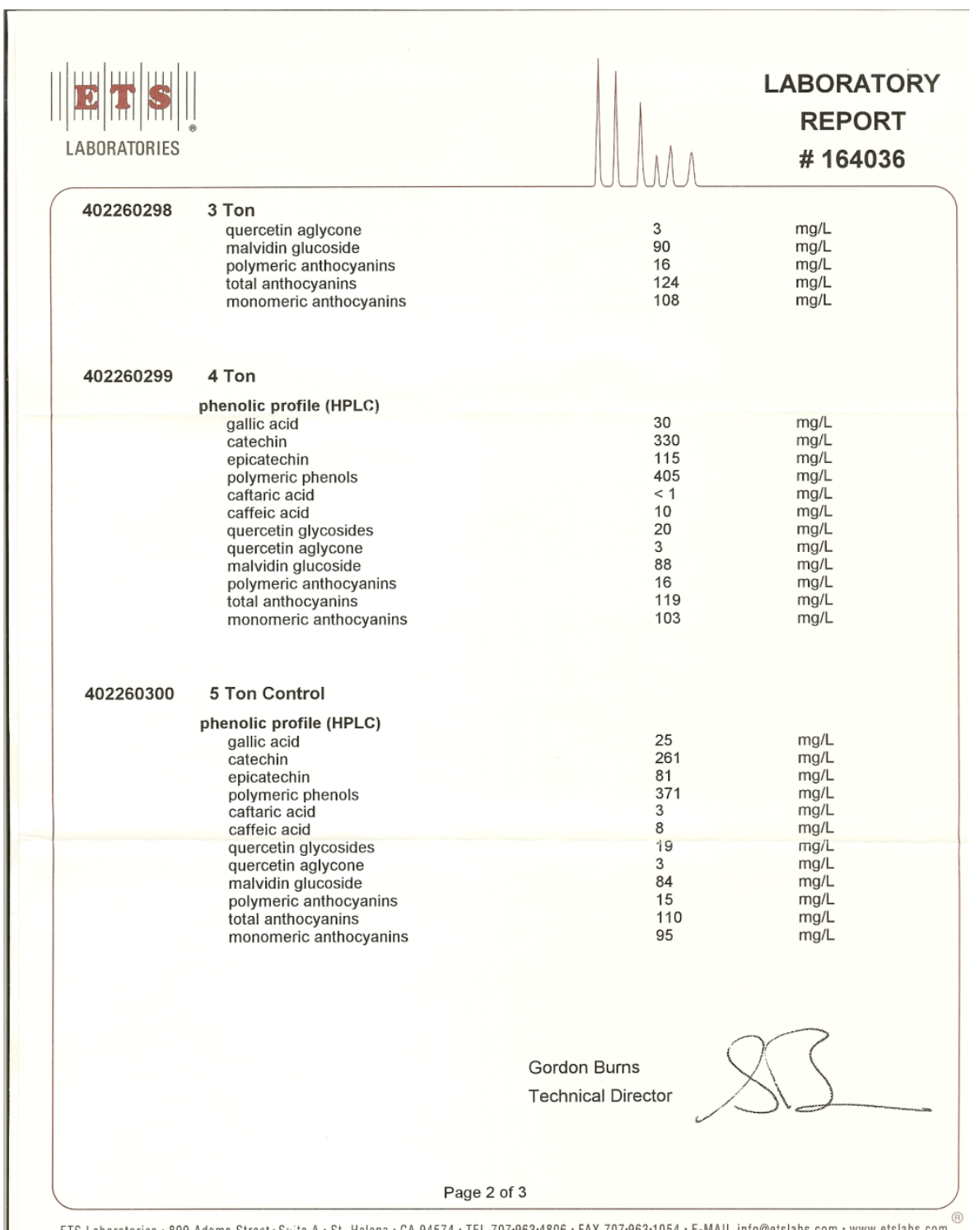

ETS Laboratories·899 Adams Street·Suite A · St. Helena· CA 94574 · TEL 707.963-4806·FAX 707.963·1054·E-MAIL info@etslabs.com·www.etslabs.com 


\section{Appendix $\mathrm{H}$}

Wine Analysis 2003 growing season 1 ton treatment compared to 5 ton control

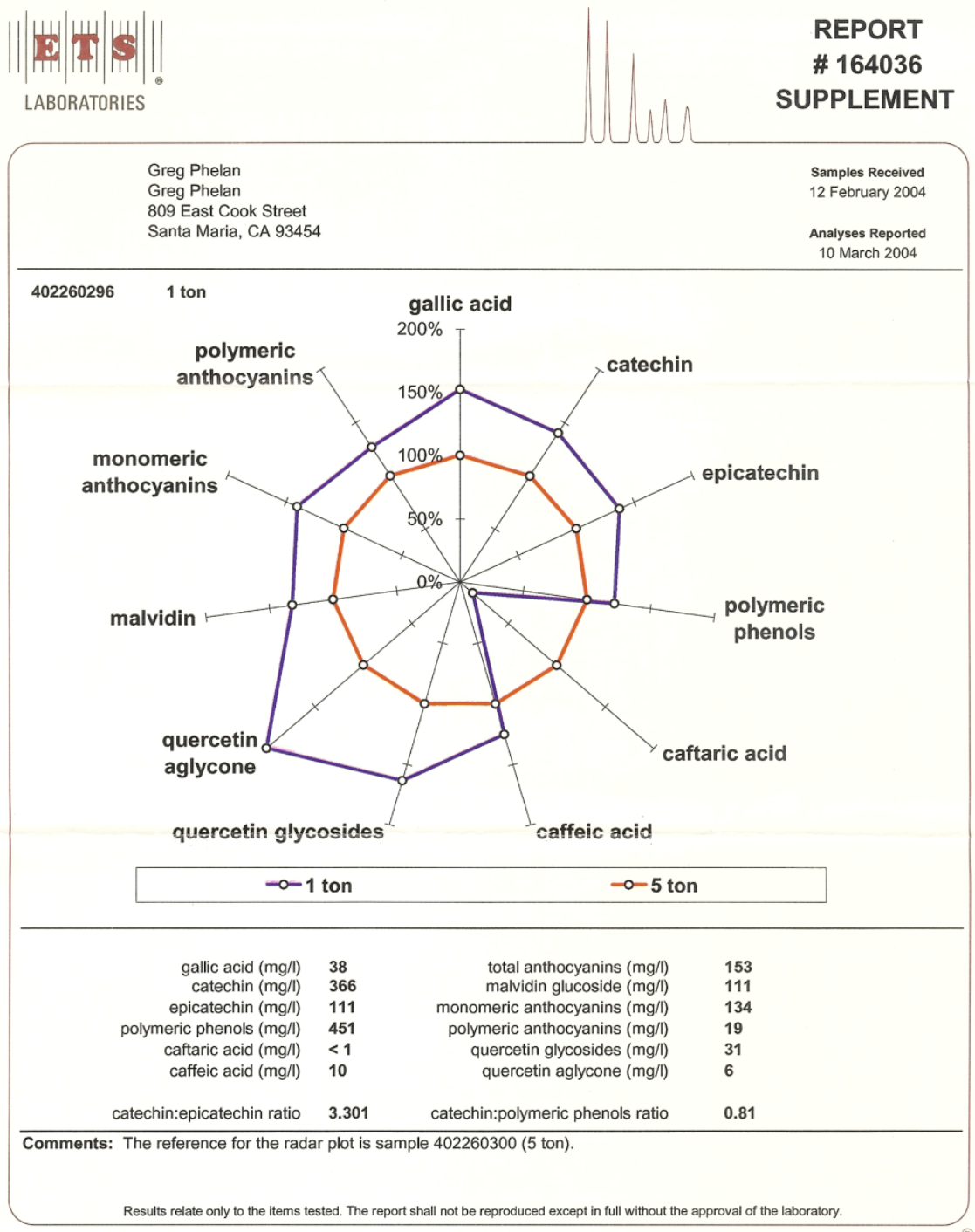

ETS Laboratories·899 Adams Street·Suite A·St. Helena · CA 94574 · TEL 707-963·4806· FAX 707-963·1054·E-MAIL info@etslabs.com·www.etslabs.com 


\section{Appendix I}

\section{Wine Analysis 2003 growing season 2 ton treatment compared to 5 ton control}

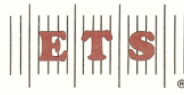

LABORATORIES

REPORT

\# 164036

SUPPLEMENT

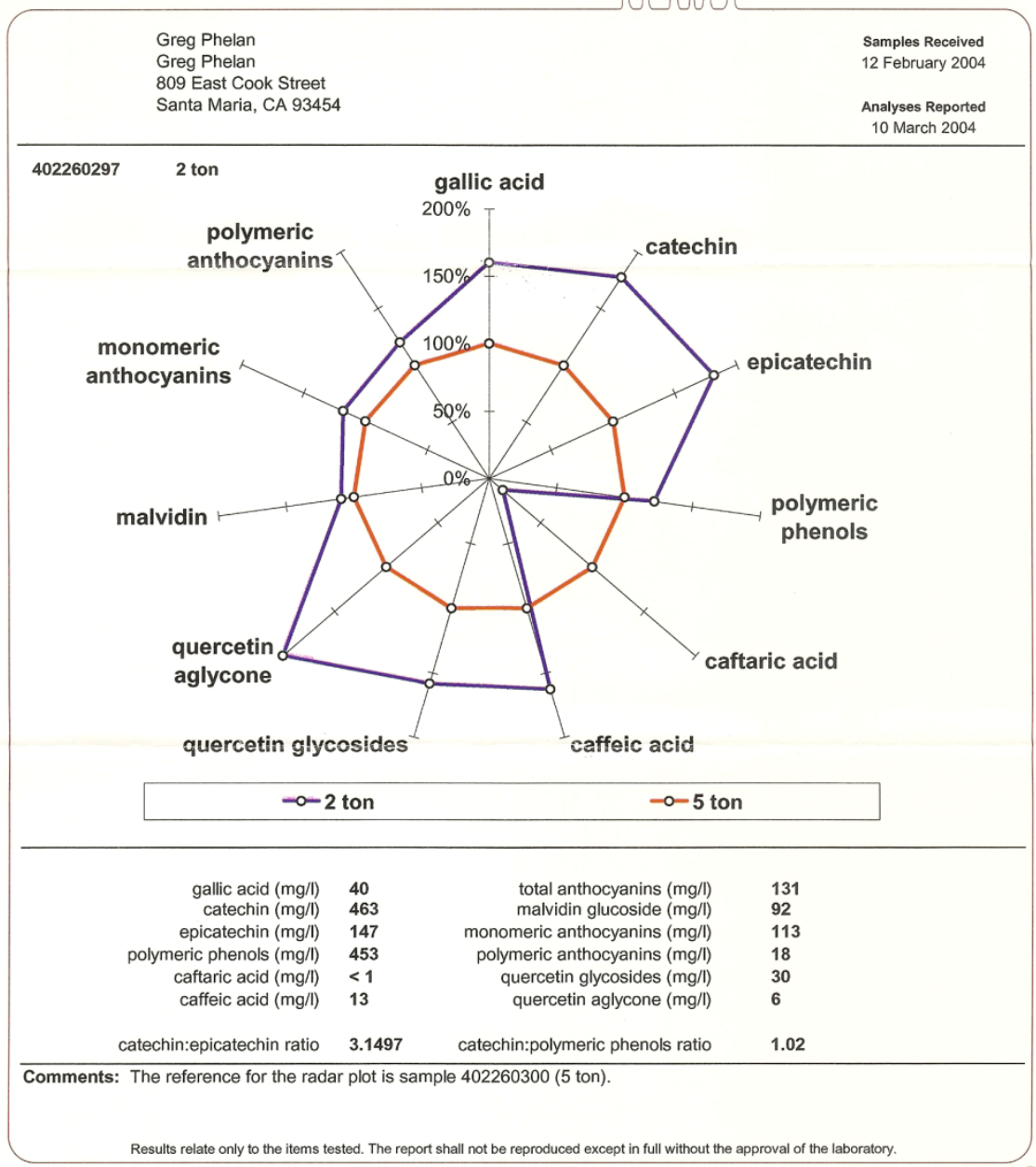

ETS Laboratories · 899 Adams Street · Suite A · St. Helena · CA 94574 · TEL 707-963-4806 · FAX 707-963-1054 · E-MAIL info@etslabs.com · www.etslabs.com 


\section{Appendix $\mathbf{J}$}

Wine Analysis 2003 growing season 3 ton treatment compared to 5 ton control

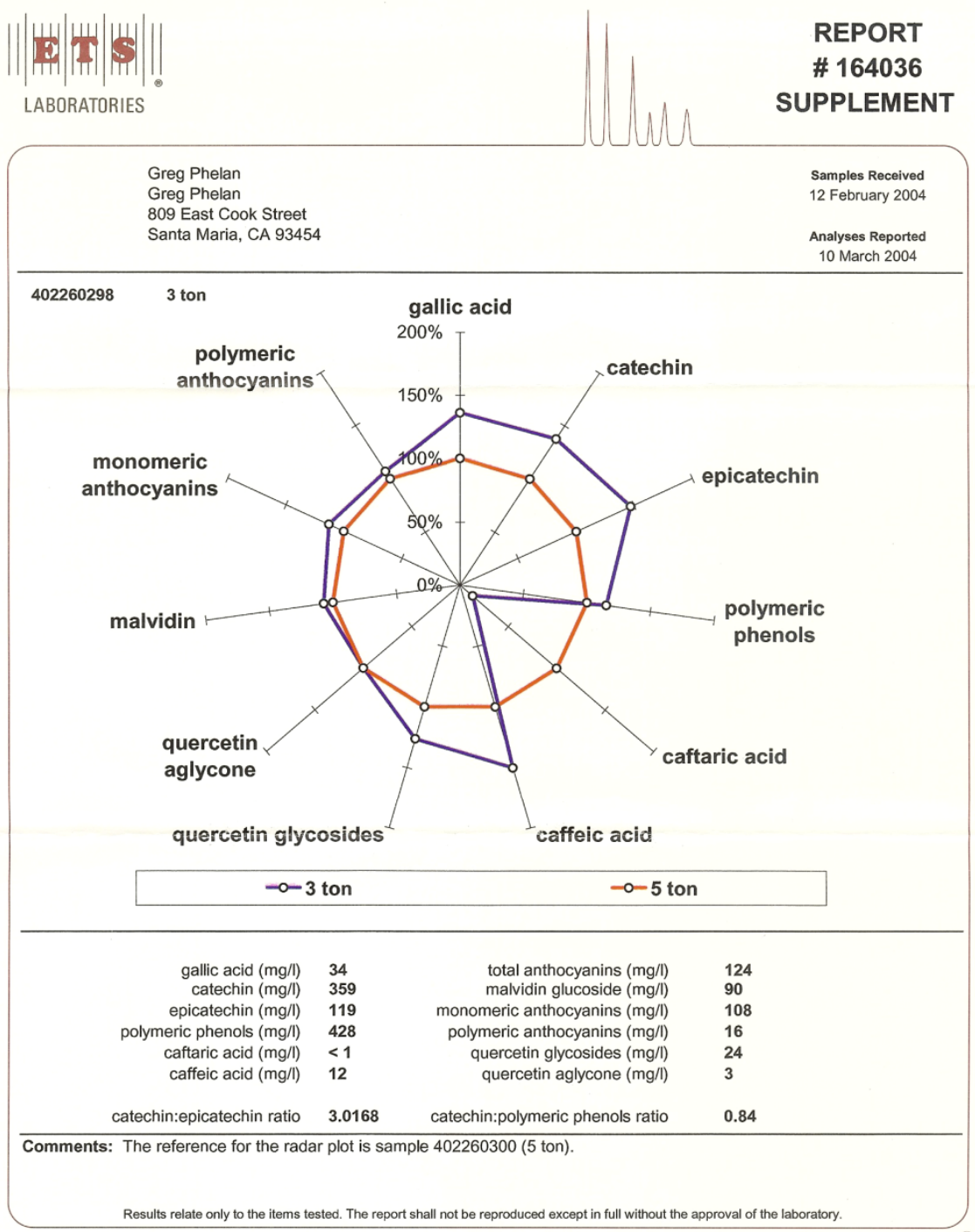

ETS Laboratories·899 Adams Street·Suite A·St. Helena · CA 94574 · TEL 707-963-4806·FAX 707-963·1054·E-MAIL info@etslabs.com·www.etslabs.com 


\section{Appendix K}

Wine Analysis 2003 growing season 4 ton treatment compared to 5 ton control

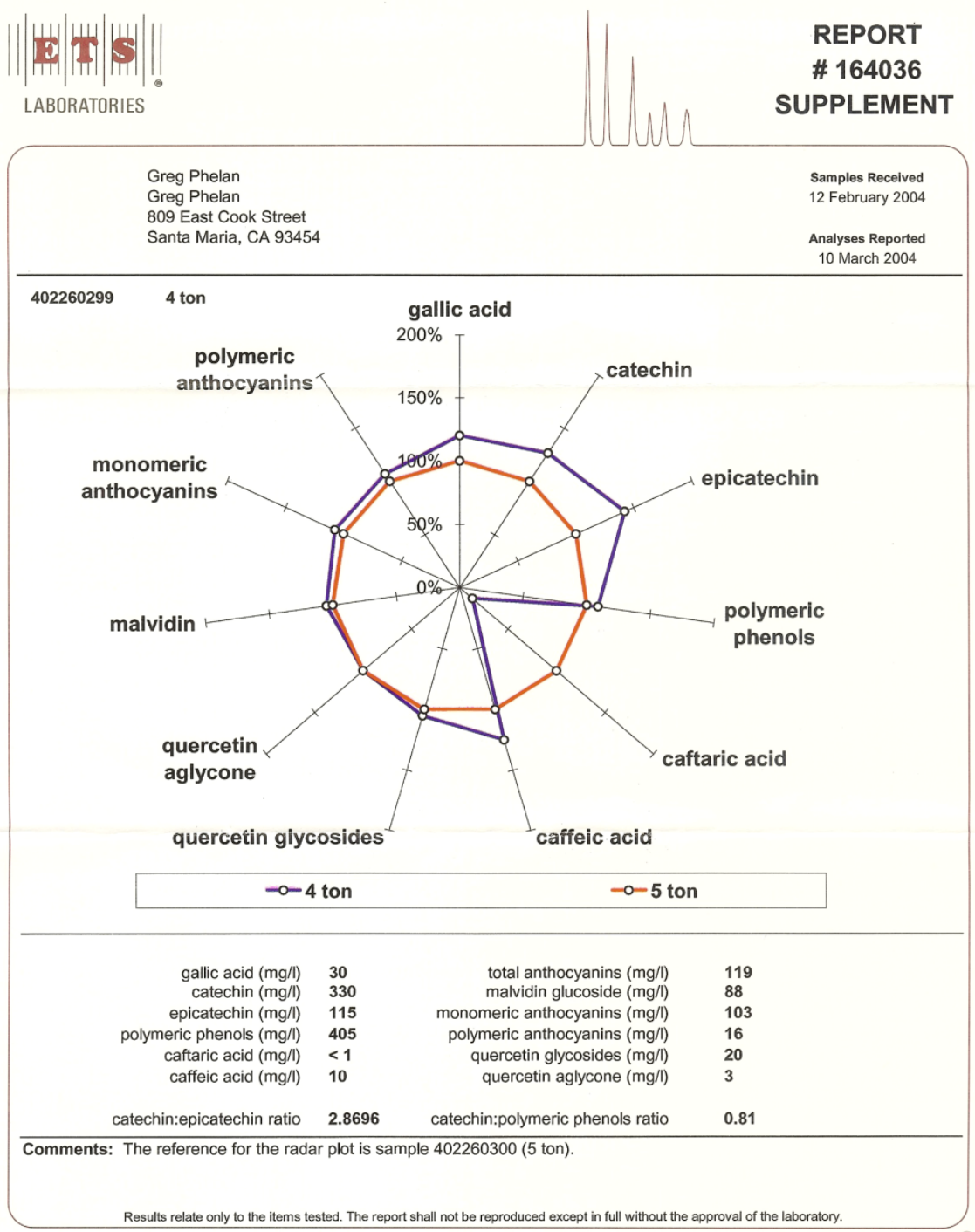

ETS Laboratories·899 Adams Street·Suite A·St. Helena·CA 94574 · TEL 707-963-4806·FAX 707-963·1054·E-MAIL info@etslabs.com·www.etslabs.com 


\section{Appendix L}

\section{Wine Analysis 2003 growing season 5 ton control}

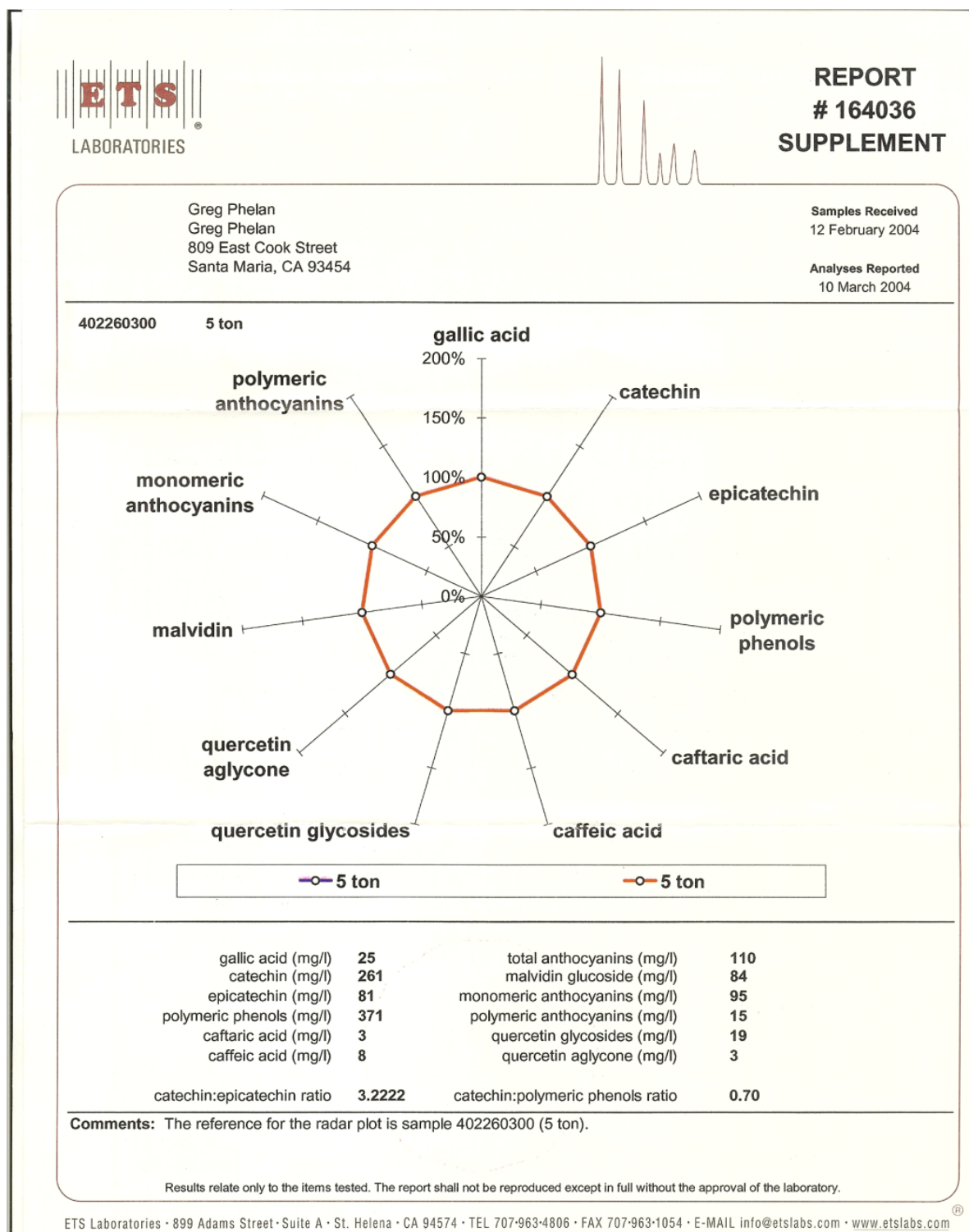




\section{Appendix M}

ANOVA Tables

General Linear Model: TA versus E/L, tons, season

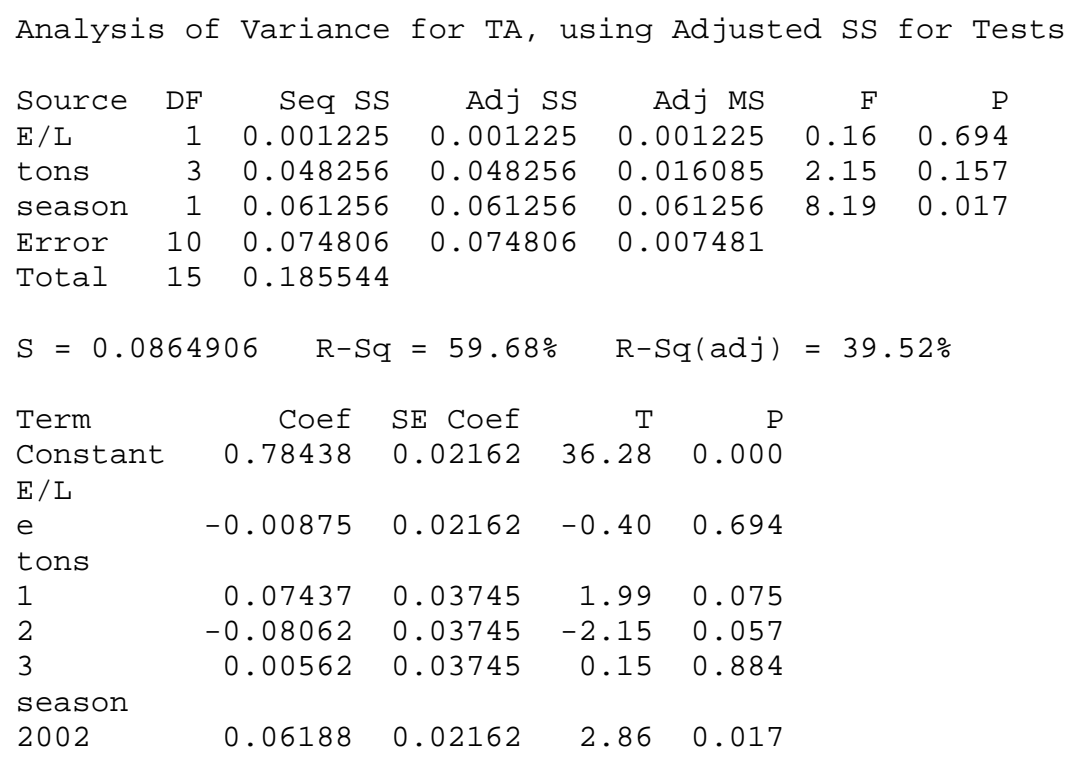




\section{Appendix N}

ANOVA Tables

General Linear Model: pH versus E/L, tons, season

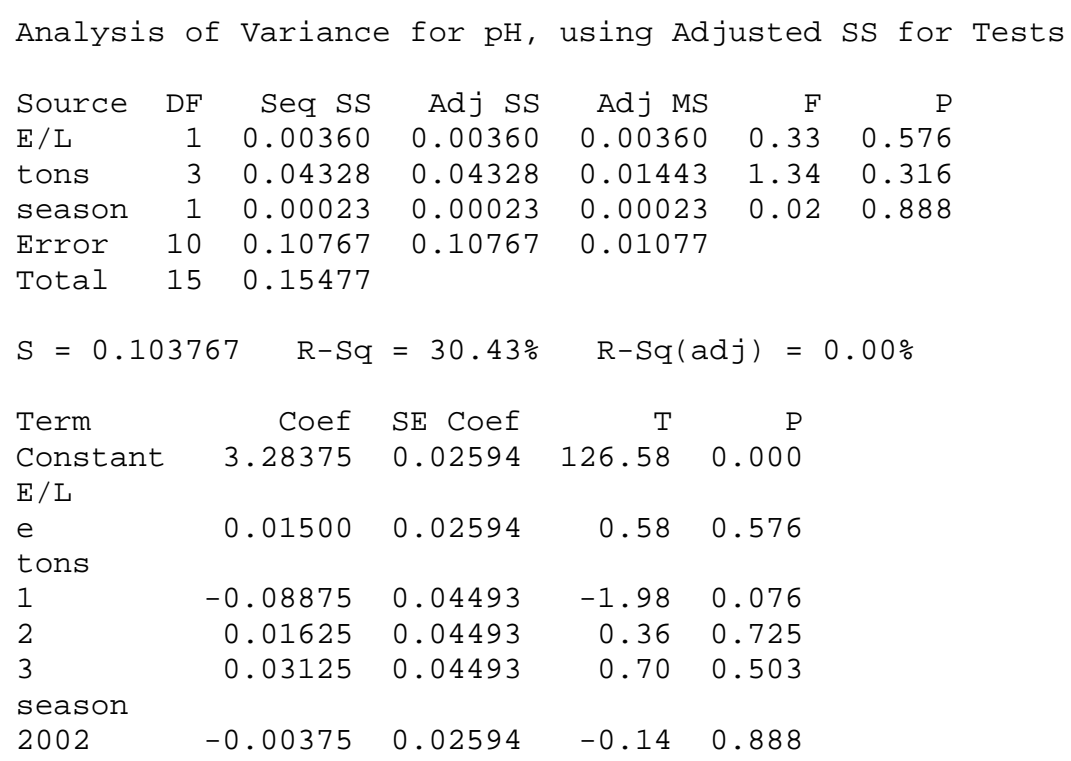




\section{Appendix $\mathrm{O}$}

ANOVA Tables

General Linear Model: Brix versus E/L, tons, season

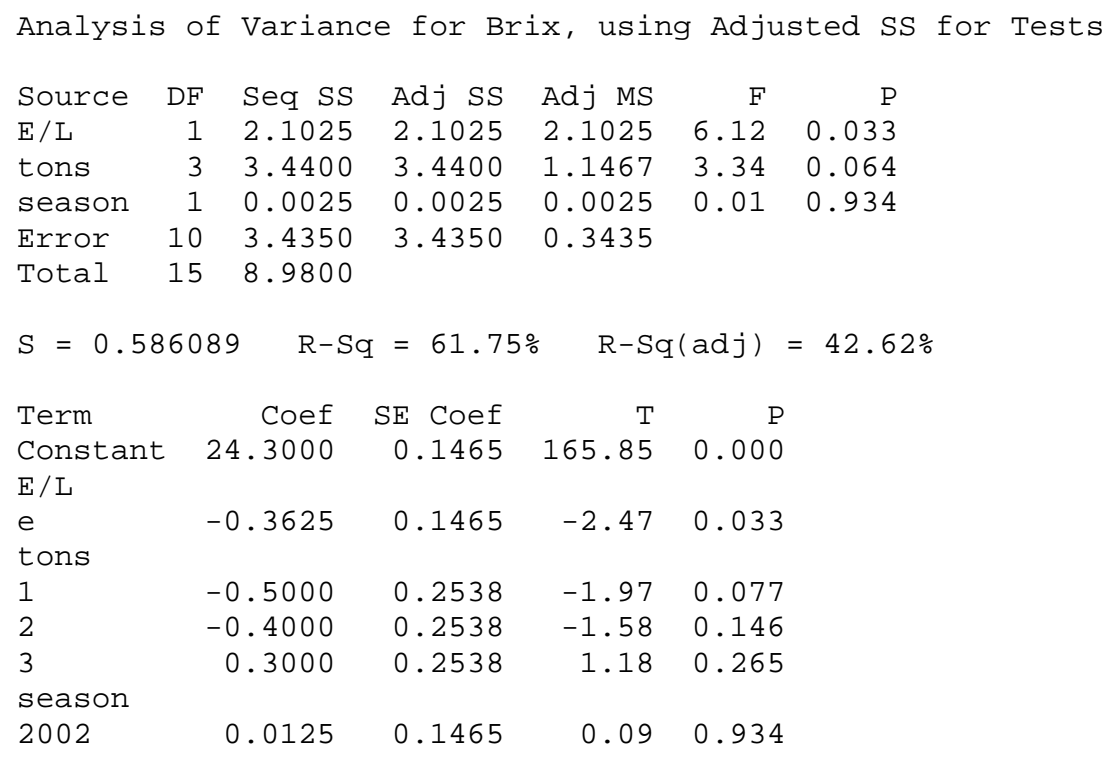


Appendix $\mathrm{P}$

ANOVA Tables

2002 growing season

General Linear Model: Caliper versus Drop Level, DD, Week, TMB

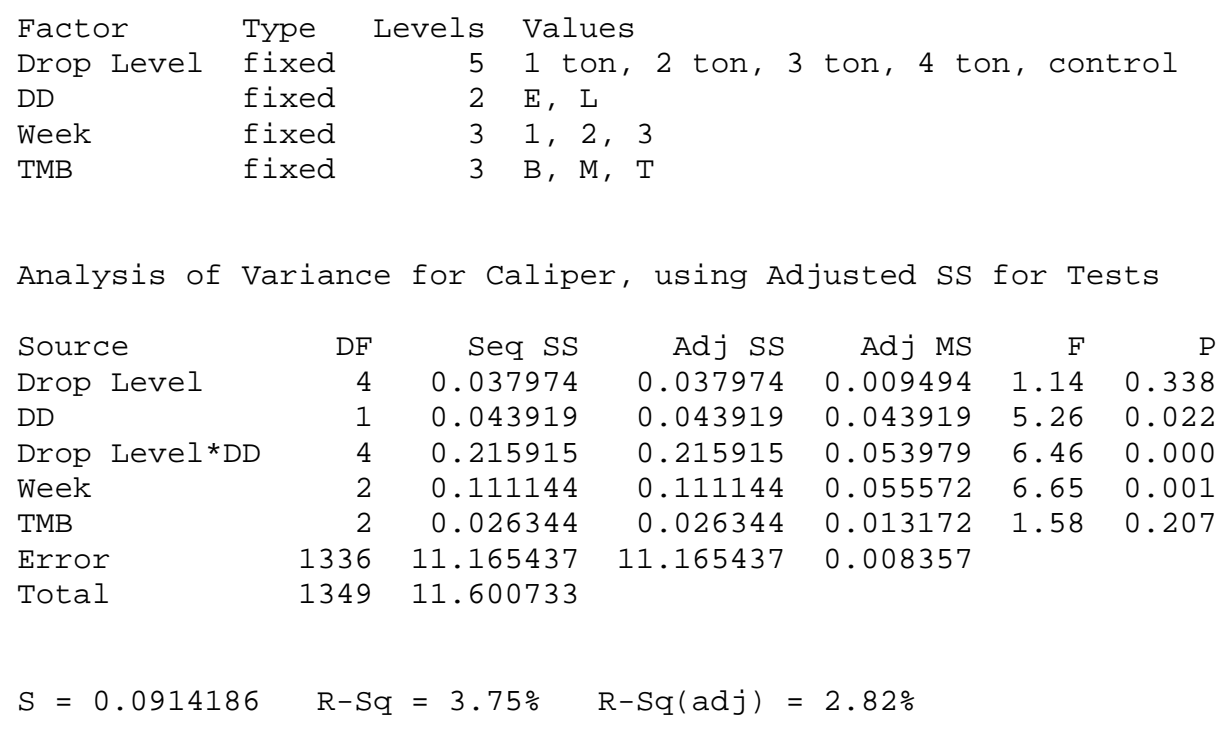




\section{Appendix Q}

ANOVA Tables

2003 growing season

General Linear Model: Caliper versus Drop Level, DD, Week, TMB

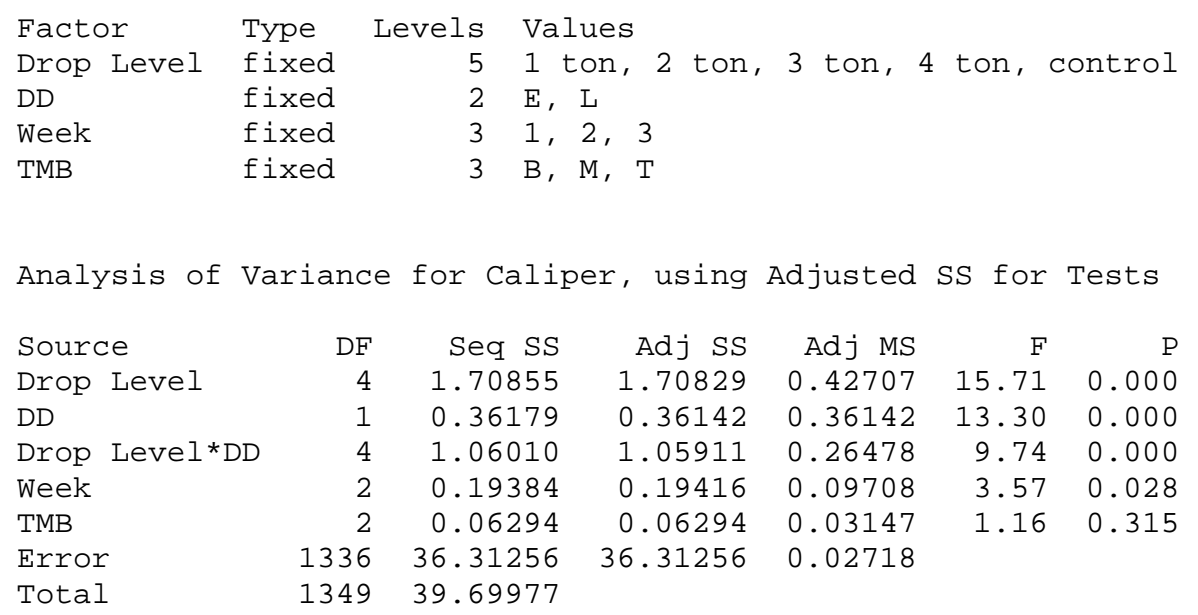




\section{Appendix R}

ANOVA Tables

General Linear Model: $\mathbf{2 0 0 2}$ growing season

\section{Avg.oz/cluster/vine versus Drop Level, Drop Time}

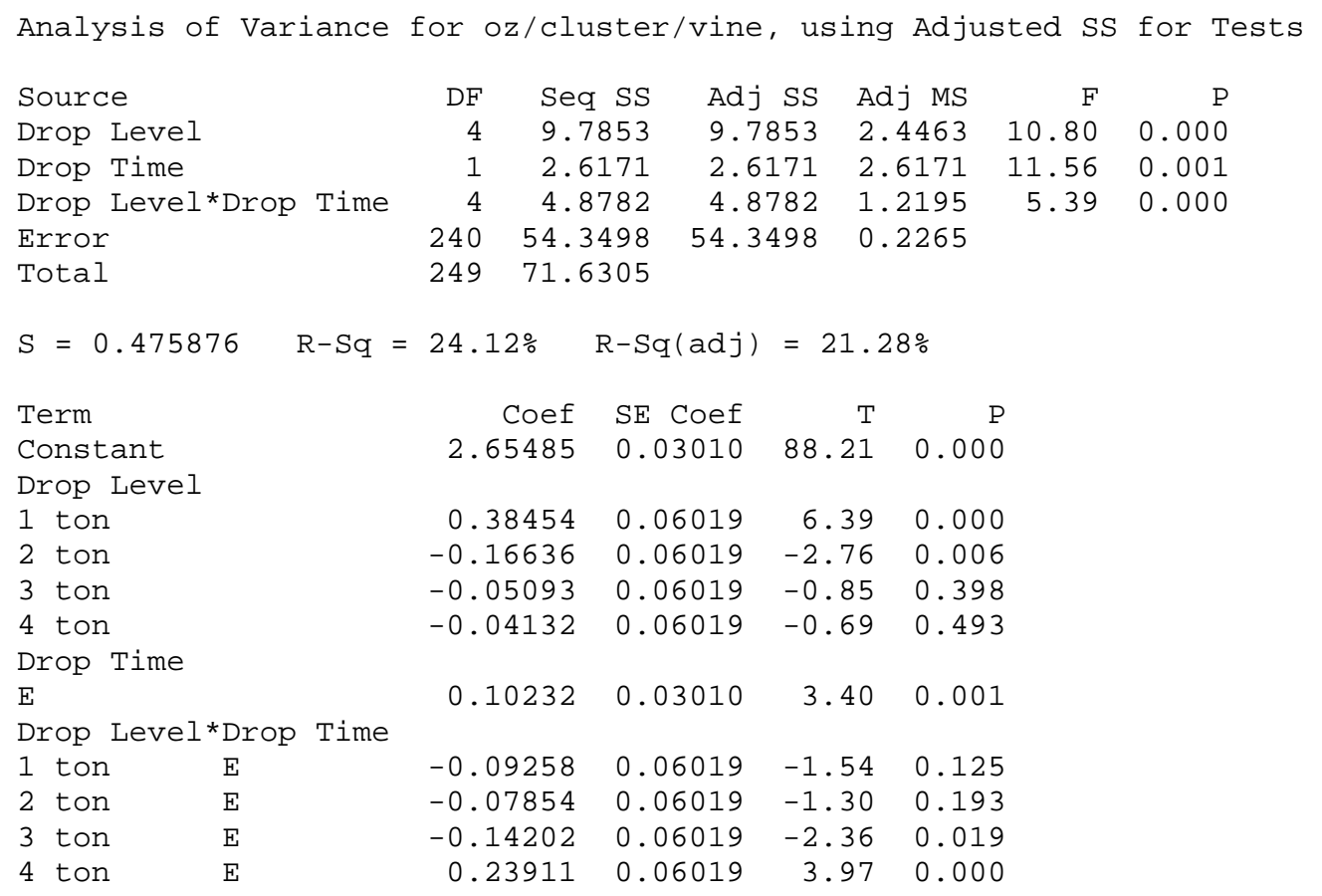




\section{Appendix S}

ANOVA Tables

General Linear Model: 2003 growing season

\section{Avg.oz/cluster/vine versus Drop Level, Drop Time}

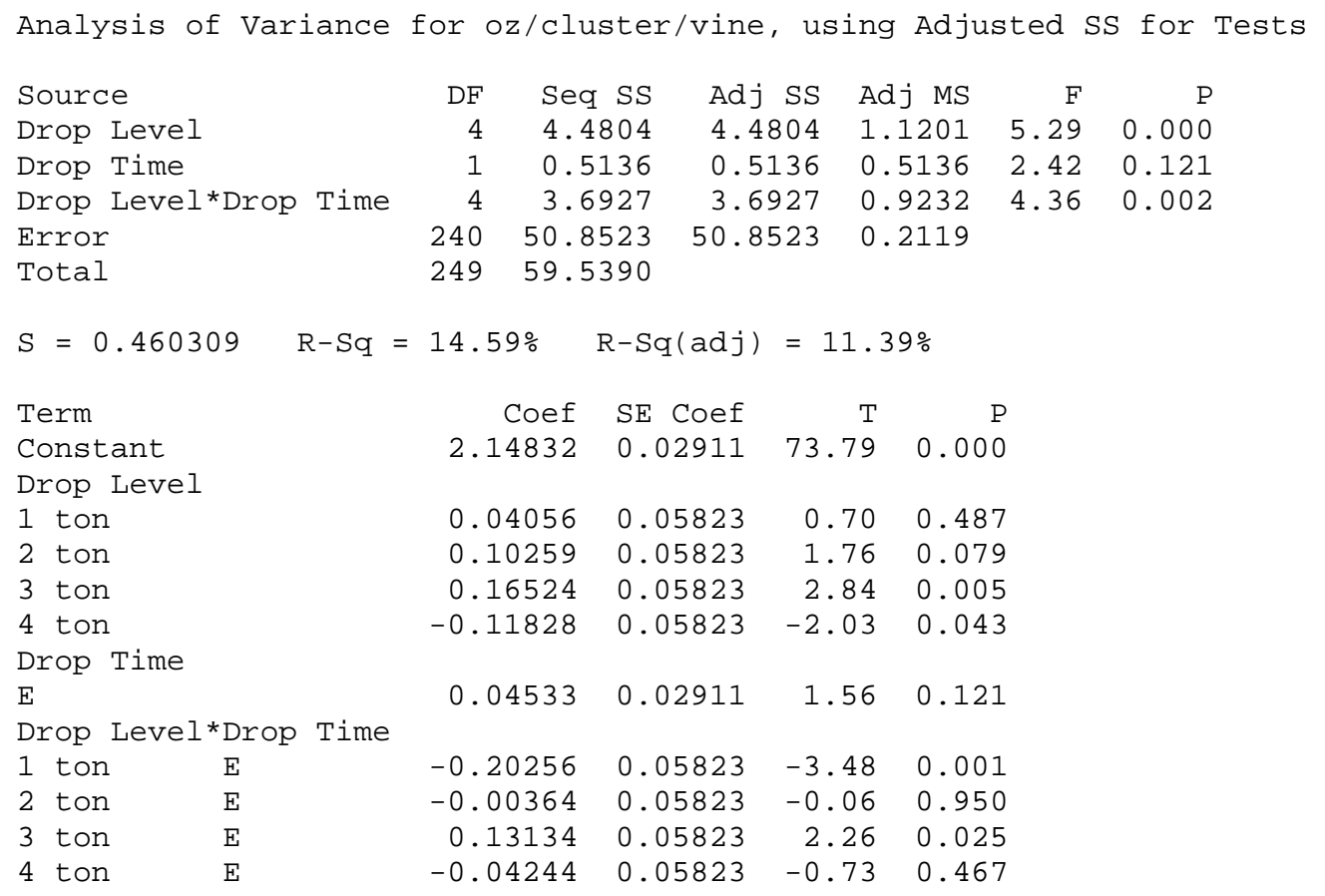




\section{Appendix T}

ANOVA Tables

\section{2 growing season}

General Linear Model: sqrt(oz/shoot/vine) versus Drop Level, Drop Time

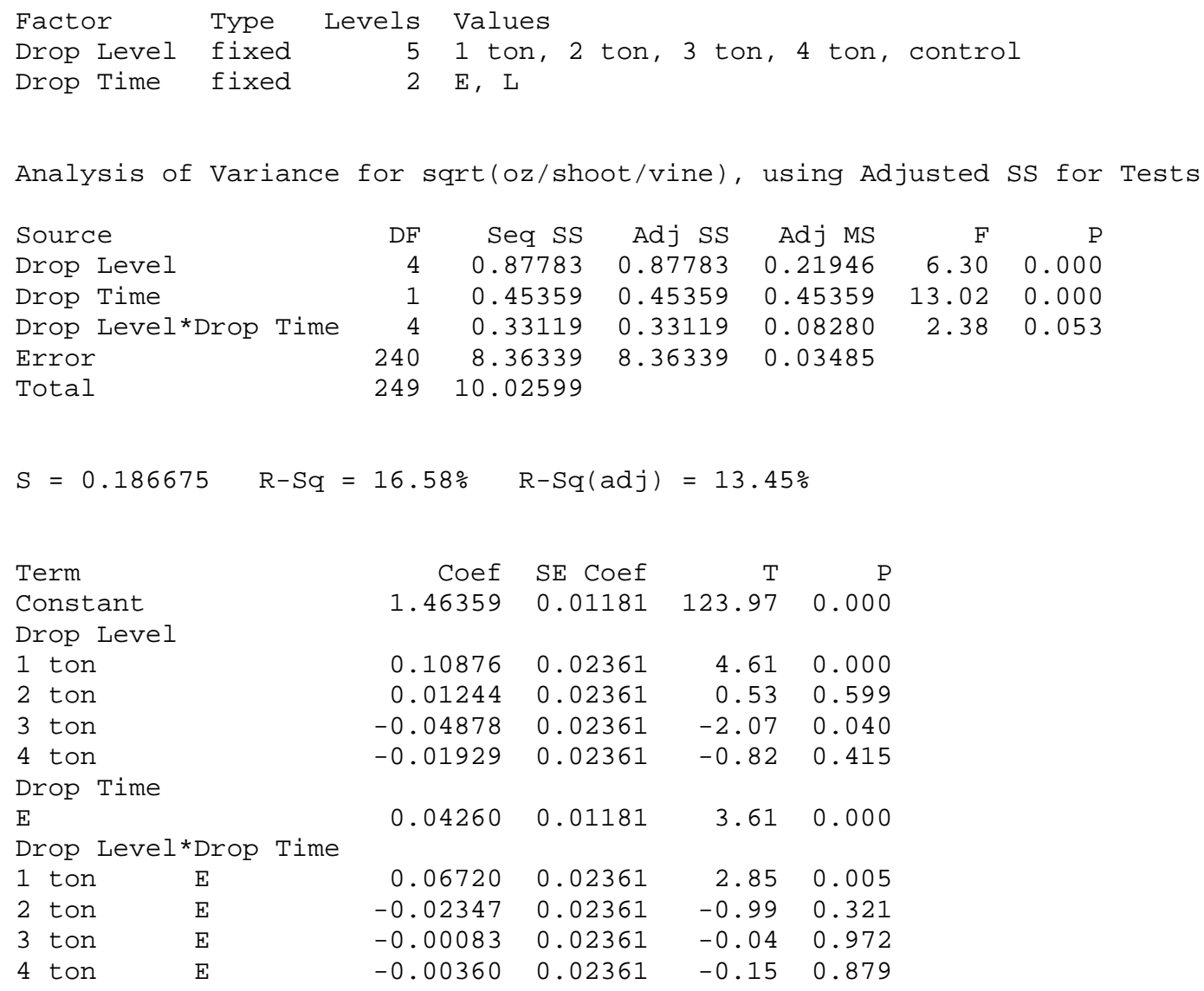




\section{Appendix U}

ANOVA Tables

2003 growing seaon

General Linear Model: sqrt(oz/shoot/vine) versus Drop Level, Drop Time

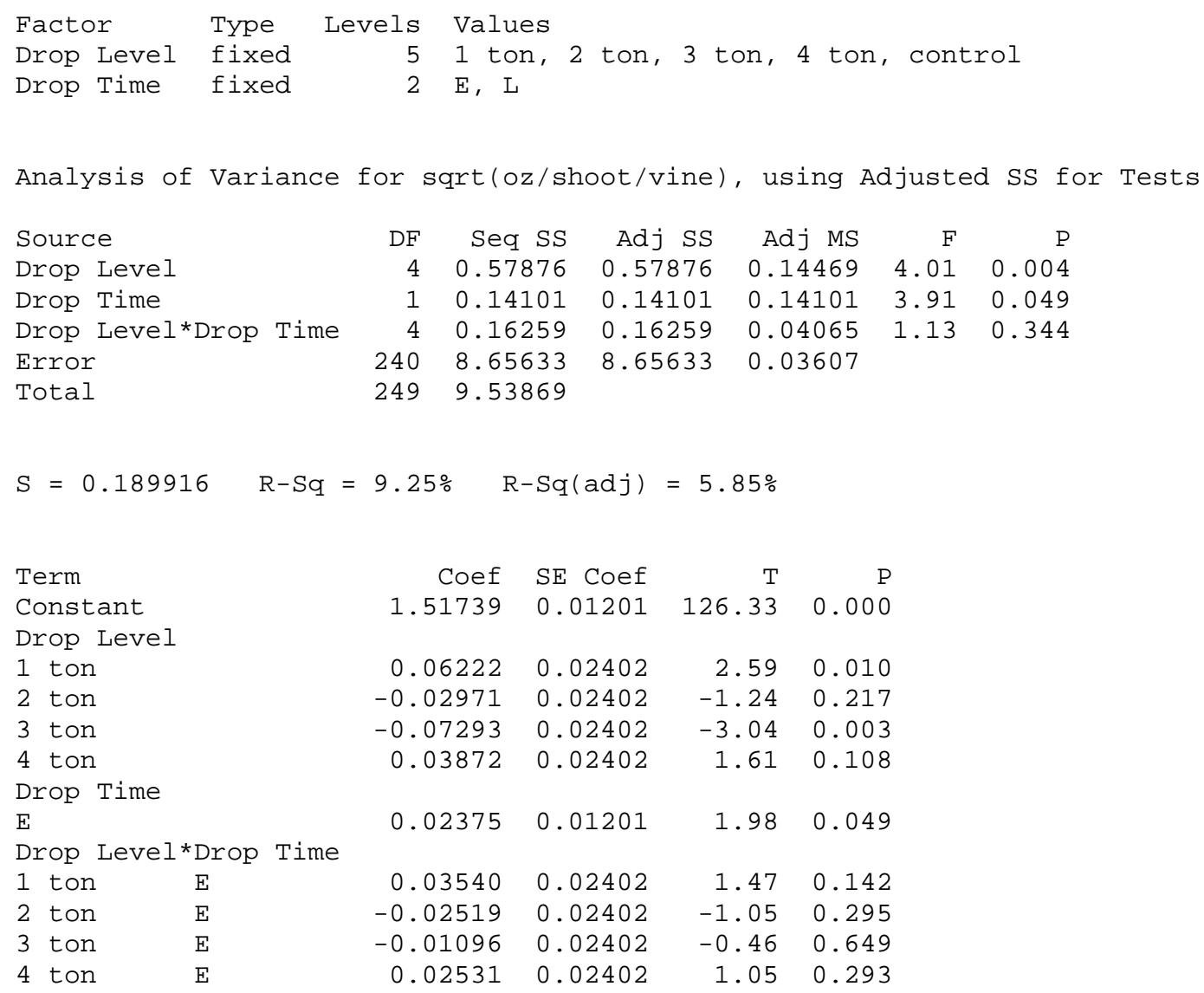




\section{Appendix V}

ANOVA Tables

General Linear Model: gallic acid versus Treatment, Year

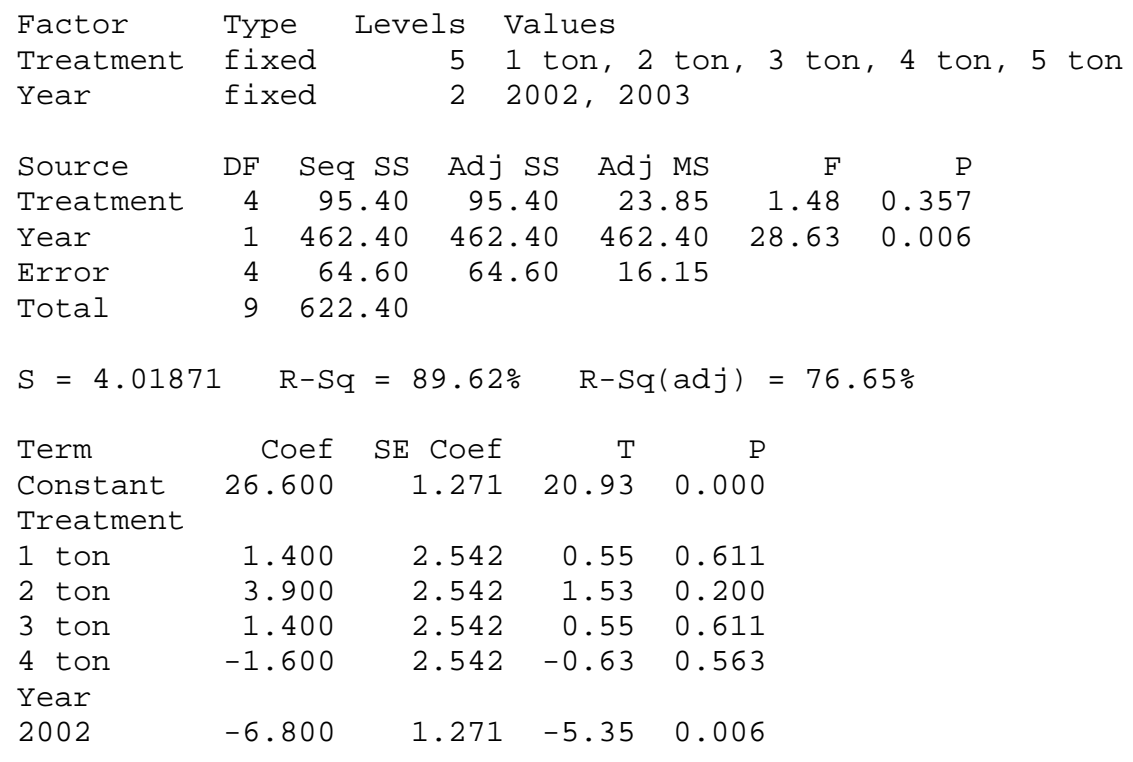


Appendix W

ANOVA Tables

General Linear Model: catechin versus Treatment, Year

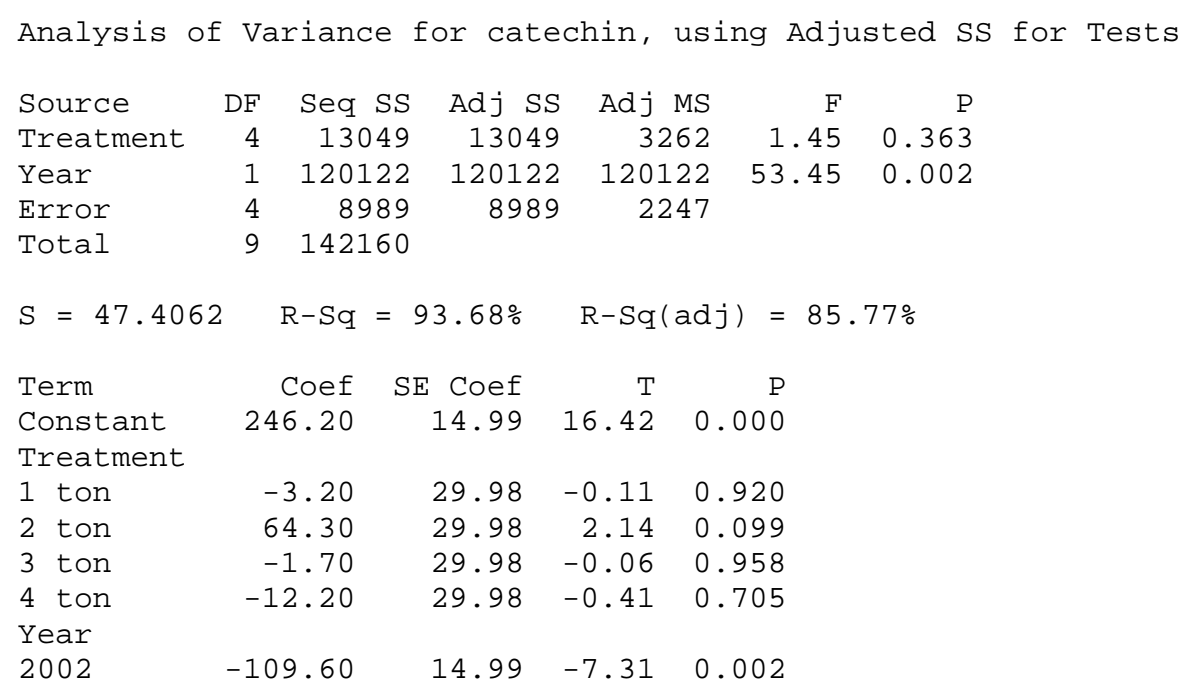




\section{Appendix X}

ANOVA Tables

General Linear Model: epicatechin versus Treatment, Year

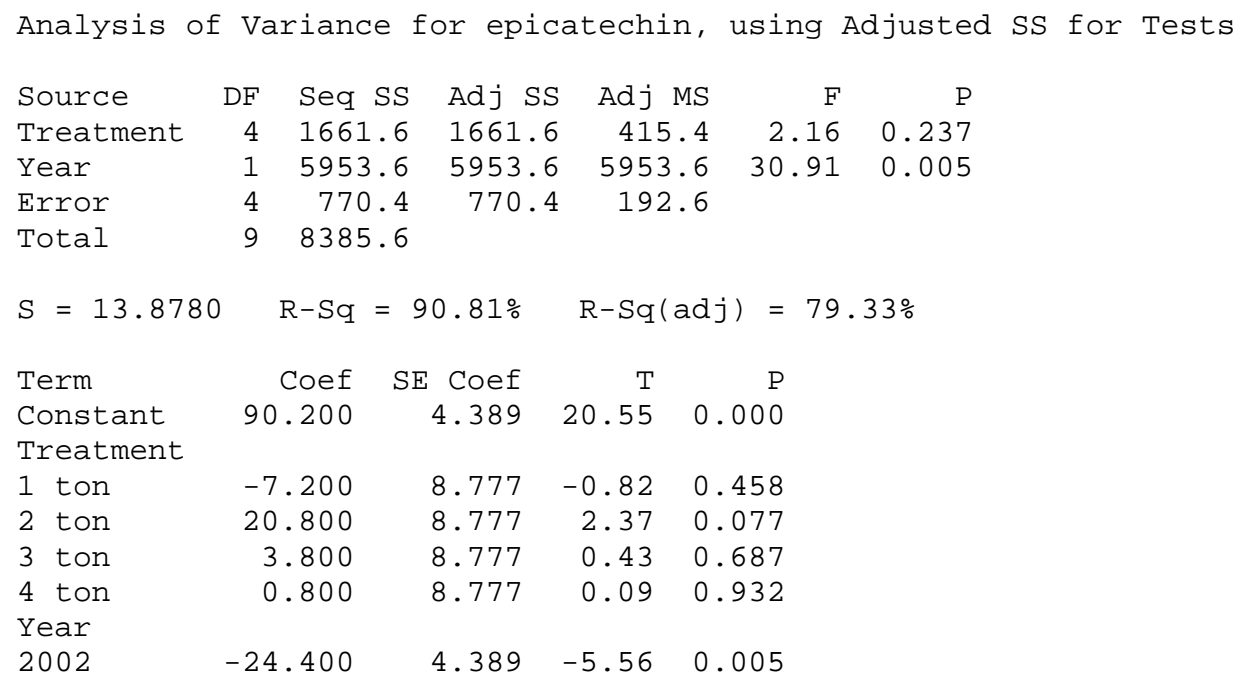




\section{Appendix Y}

ANOVA Tables

General Linear Model: polymeric phenols versus Treatment, Year

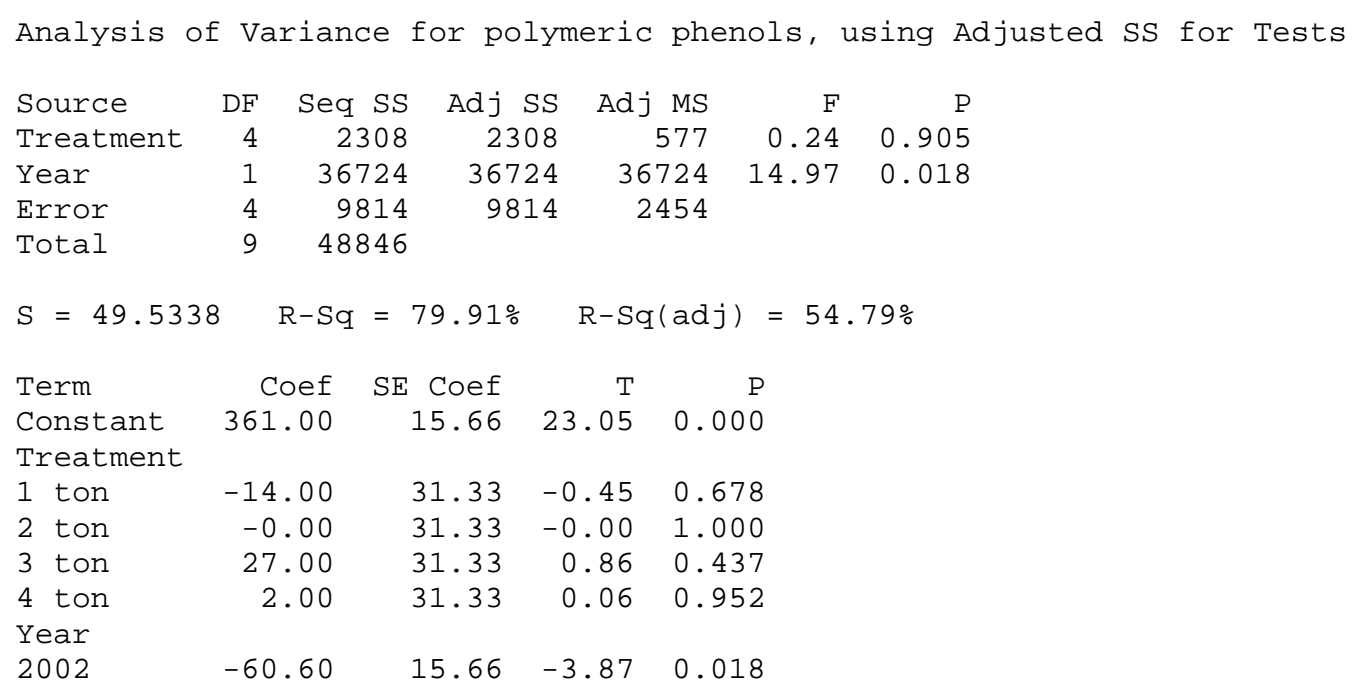




\section{Appendix Z}

ANOVA Tables

General Linear Model: caftaric acid versus Treatment, Year

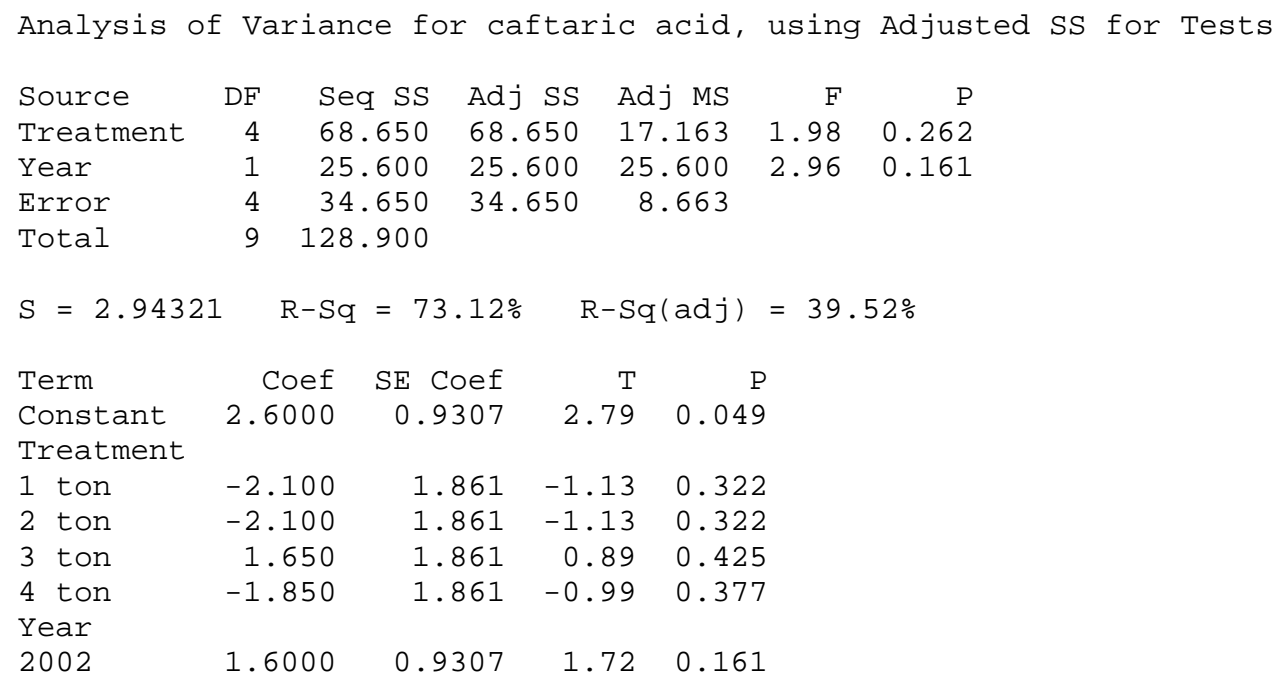


ANOVA Tables

Appendix AA

General Linear Model: caffeic acid versus Treatment, Year

\begin{tabular}{|c|c|c|c|c|c|c|c|c|}
\hline Factor & Type & Valu & & & & & & \\
\hline Treatment & fixed & 1 to & $n, 2$ ton, & 3 tor & $n, 4$ ton, & $5 t$ & ton & \\
\hline Year & fixed & 2002 & 2003 & & & & & \\
\hline Analysis of & Variance & or caffe & ic acid, & using & Adjusted & SS & for & Tests \\
\hline Source & Seq SS & Adj $S S$ & Adj MS & F & $\mathrm{P}$ & & & \\
\hline Treatment & 66.600 & 66.600 & 16.650 & 2.85 & 0.168 & & & \\
\hline Year & 8.100 & 8.100 & 8.100 & 1.38 & 0.305 & & & \\
\hline Error & 23.400 & 23.400 & 5.850 & & & & & \\
\hline Total & 98.100 & & & & & & & \\
\hline$S=2.41868$ & $\mathrm{R}-\mathrm{Sq}=$ & $6.15 \%$ & $R-S q(\operatorname{adj})$ & $=46$. & $.33 \%$ & & & \\
\hline
\end{tabular}




\section{Appendix BB}

ANOVA Tables

General Linear Model: quercetin glycosides versus Treatment, Year

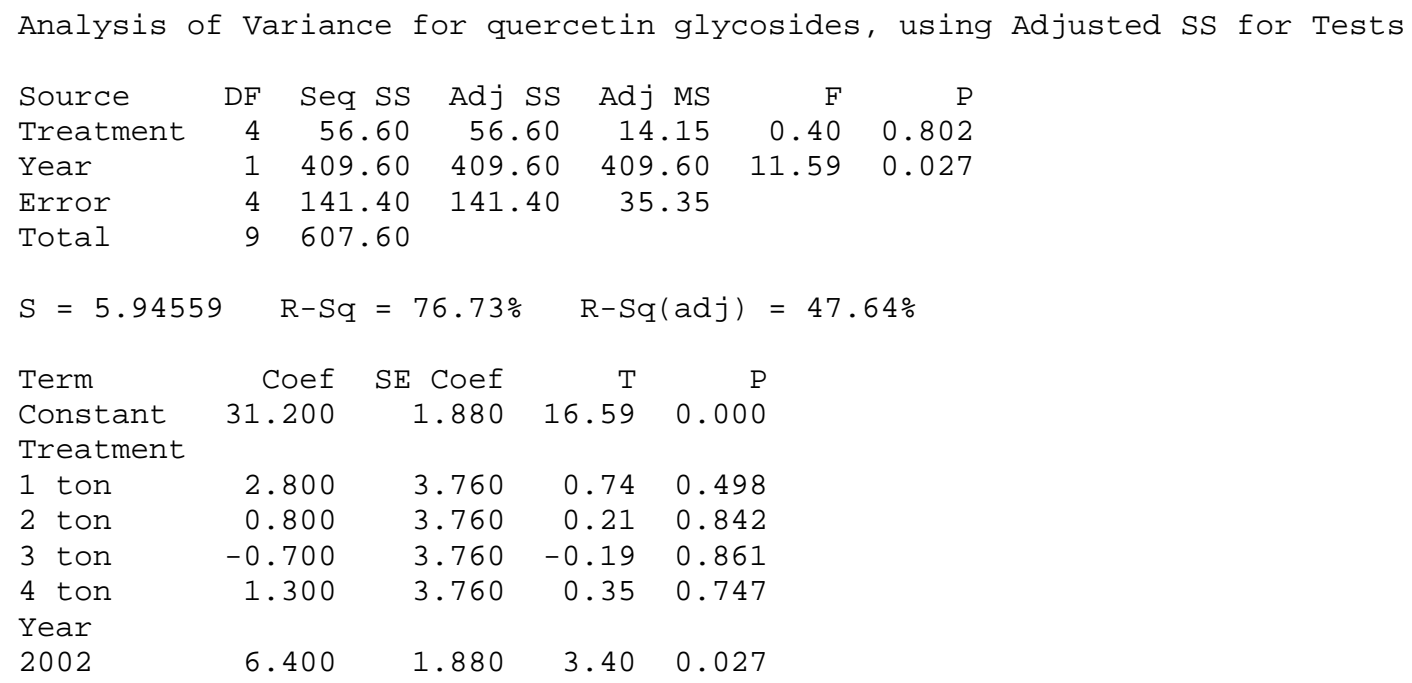


Appendix CC

ANOVA Tables

\section{General Linear Model: quercetin aglycone versus Treatment, Year}

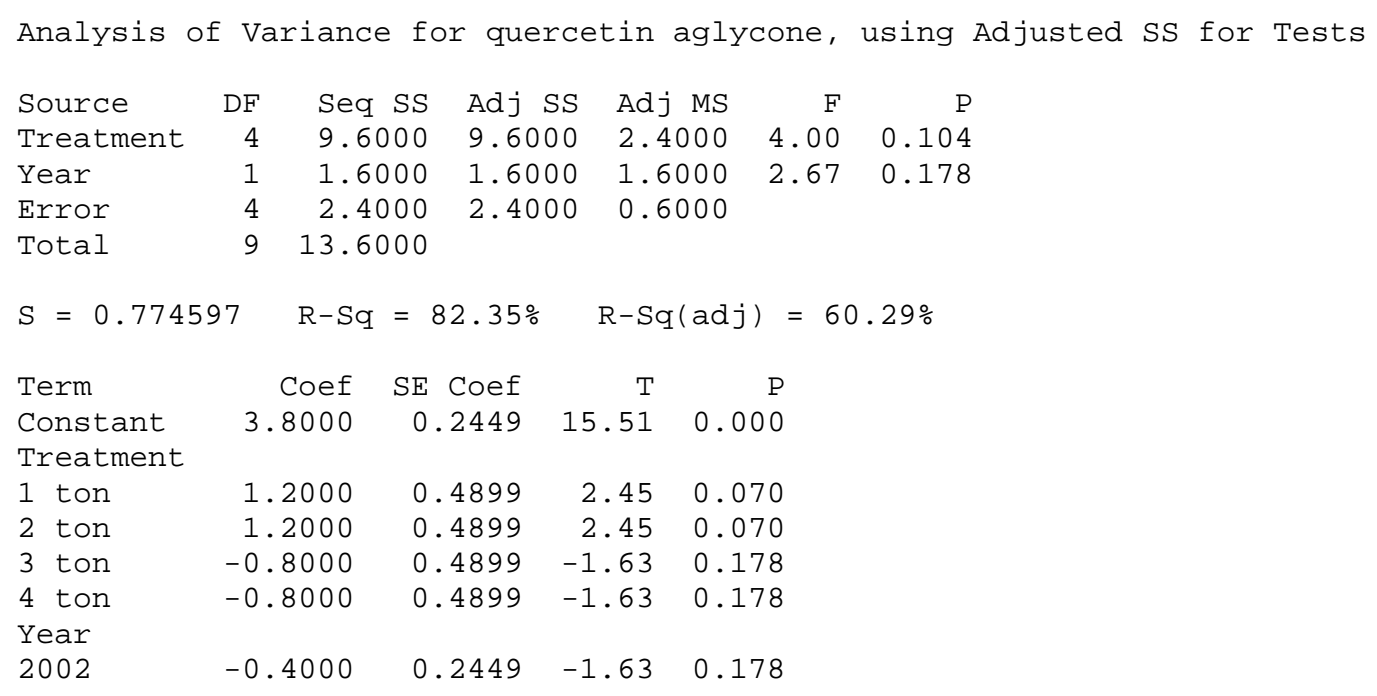


Appendix DD

ANOVA Tables

\section{General Linear Model: malvidin glucoside versus Treatment, Year}

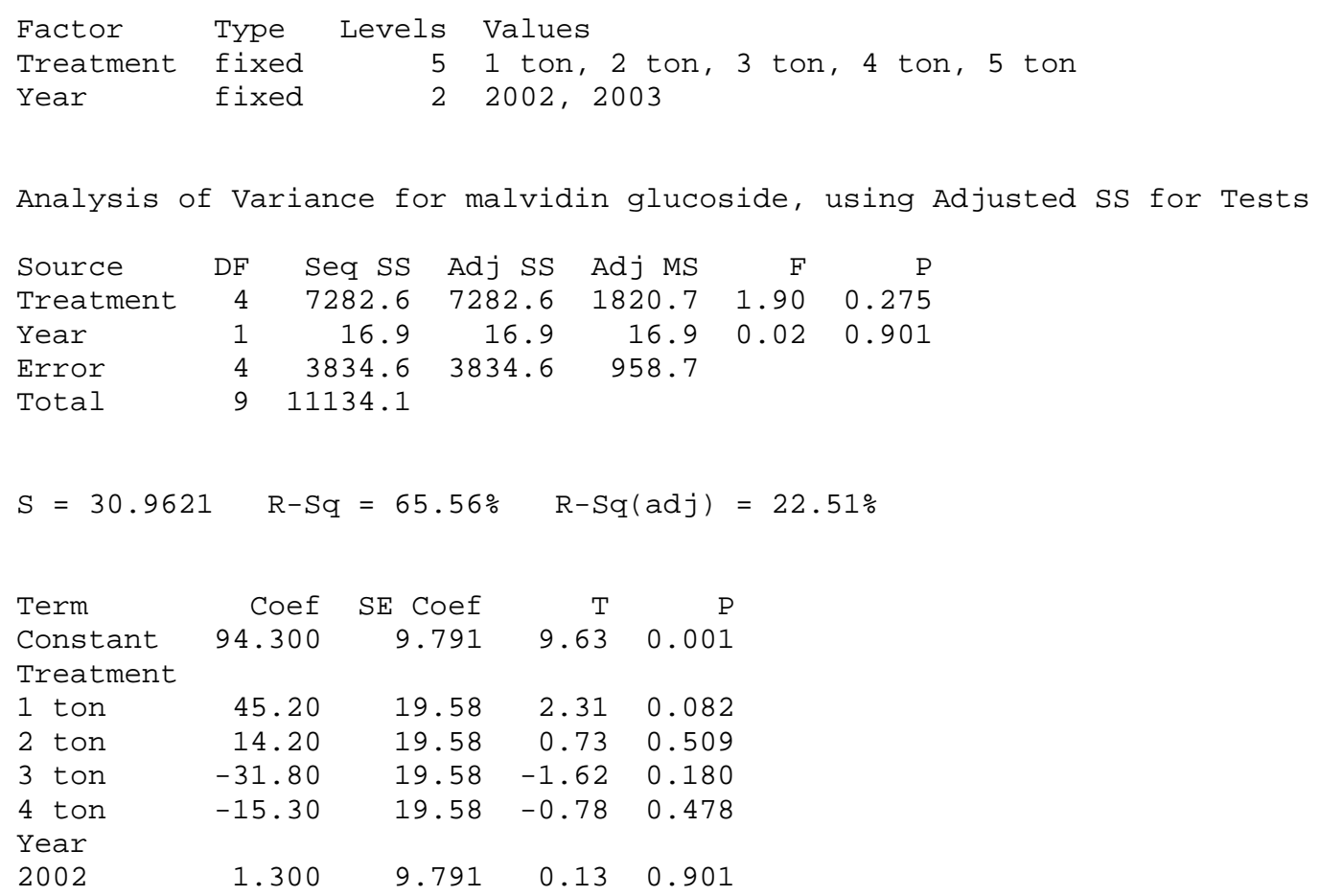




\section{Appendix EE}

ANOVA Tables

\section{General Linear Model: polymeric anthocyanins versus Treatment, Year}

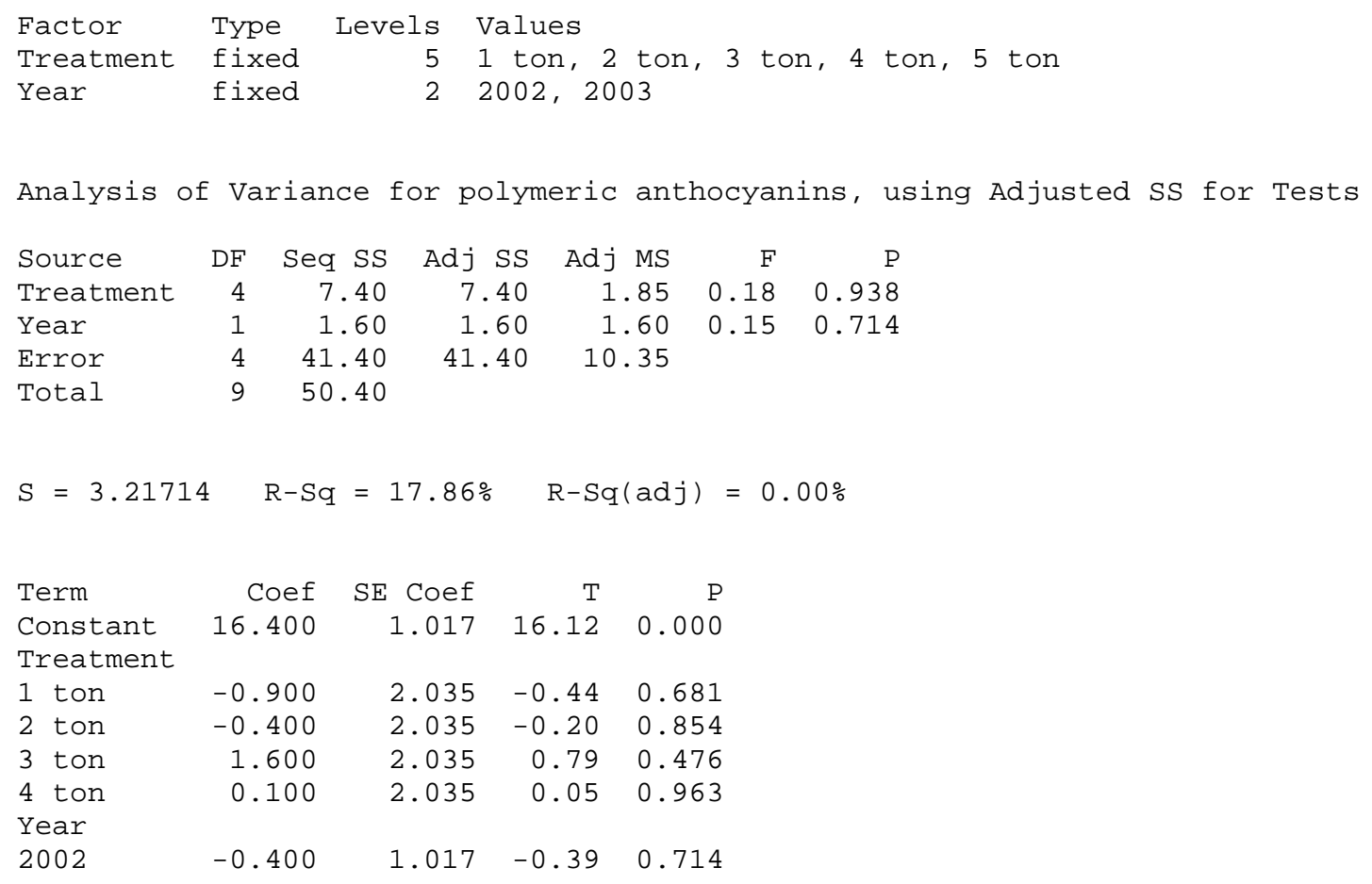




\section{Appendix FF}

ANOVA Tables

\section{General Linear Model: total anthocyanins versus Treatment, Year}

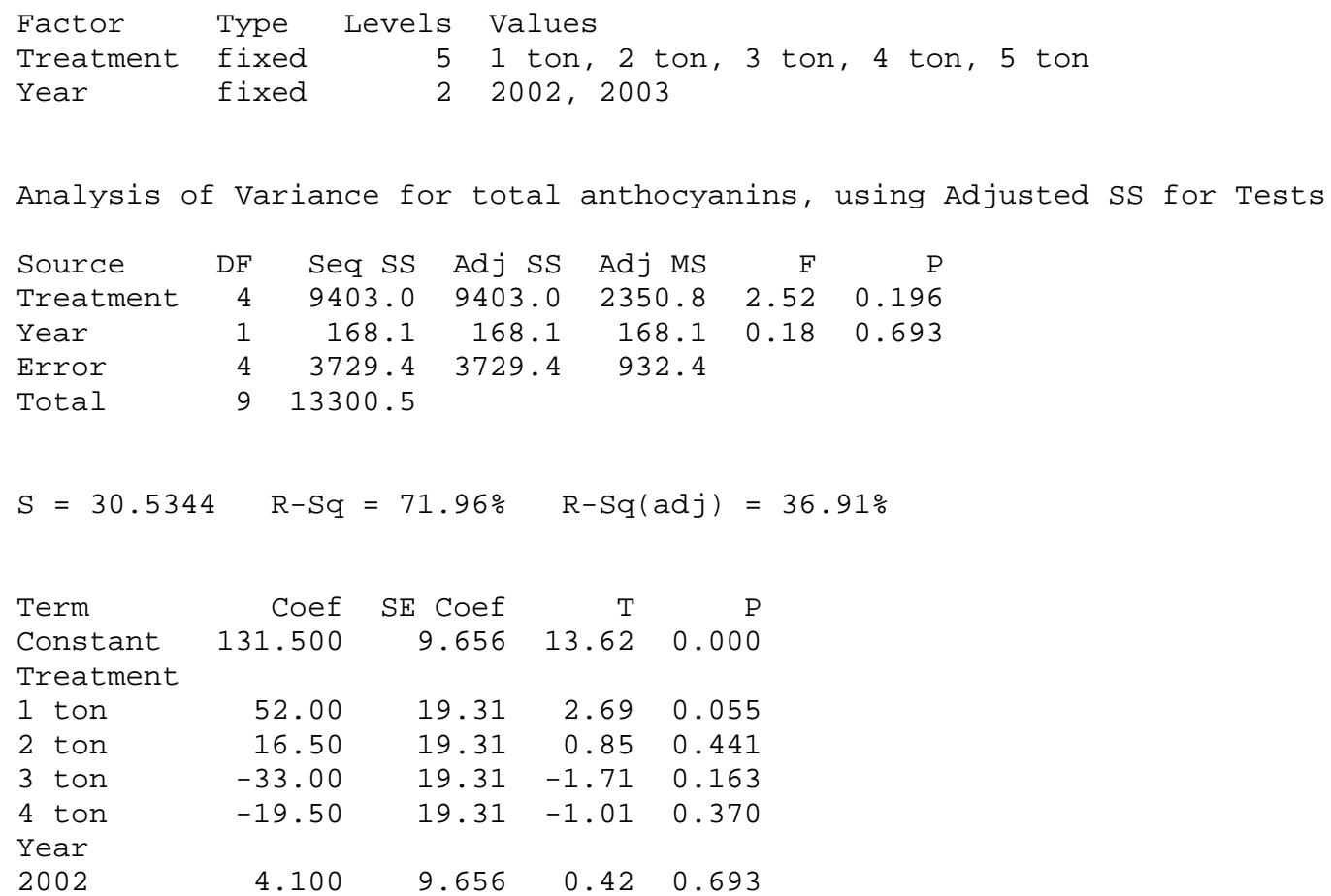




\section{Appendix GG}

ANOVA Tables

\section{General Linear Model: monomeric anthocyanins versus Treatment, Year}

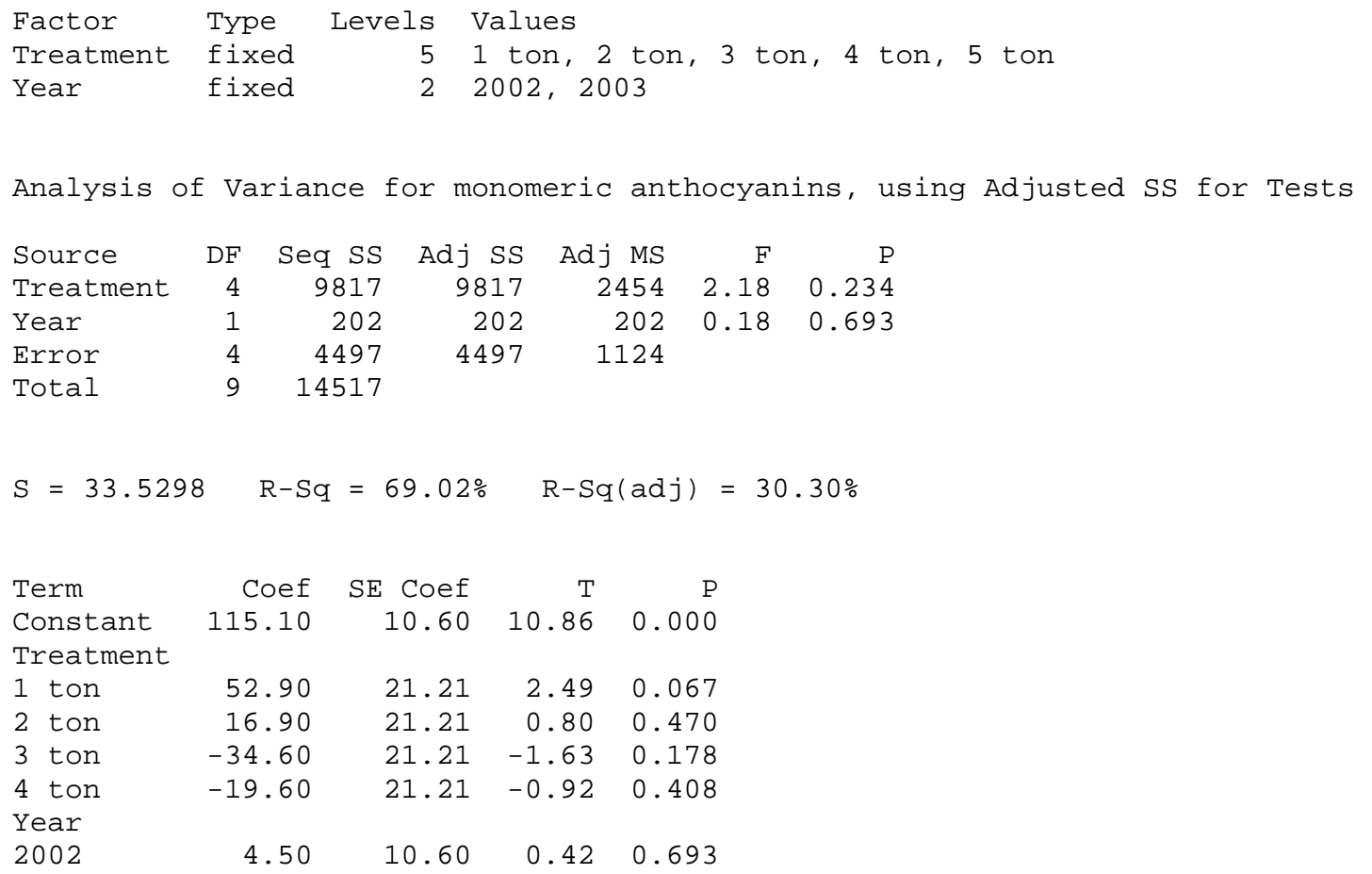

